\title{
PROCEEDINGS OF THE FORTY-SIXTH ANNUAL MEETING OF \\ THE AMERICAN SOCIETY FOR CLINICAL INVESTIGATION HELD IN ATLANTIC CITY, N. J., MAY 4, 1954
}

\author{
Presidential. Address
}

BY CARL V. MOORE

The size of this audience, year after year, is an impressive testimonial to the vital and still growing interest in clinical investigation. To a large extent it exemplifies the great desire of a constantly increasing number of young men to make significant medical contributions of their own, to have full-time careers in academic medicine. As long as this trend continues, one can look to the future with confidence and keen anticipation that our understanding and control of disease will continue to become more complete. The young clinical investigator, however, is confronted with ever increasing competition for the most desirable academic positions, with complex duties that often provide him too little time for creative thought, and with the temptation to seek more money for his laboratory than he can wisely use. These are factors which may eventually dampen enthusiasm or otherwise adversely influence clinical investigation so as to reverse the trend.

\section{I}

It has been traditional for young men to start out with the conviction that the only academic jobs really worth having are those as chief of the service or as chairman of the department. We have surrounded those positions with an aura, a sense of reverence which has at least the faint aroma of hero worship. I have heard house officers and research fellows discuss repeatedly the fact that these coveted jobs have increased very little in number while the men who seek them have grown to the size of a small army. They feel that our frontiers are gone, our era of expansion over so that here, as in many European countries excellent men will have to turn away from universities in increasing numbers because opportunities have not kept pace with interest. I would like to argue that the reverse is true, that opportunities have multiplied. To accept and believe this statement, however, we need to keep values straight and not worry about titles.

As faculties have been expanded during the past ten or fifteen years, jobs have been created that are really better than the one the Chairman has. He sets policy, to be sure, is regarded with more esteem by the community, and receives the largest salary; but with the expansion he has had more and more administrative detail, more committee meetings to suffer through, more responsib:'ity to make certain that his associates have what they need, and less free time for his own scientific interests. By contrast, the other one or two professors in his department, the associate and the assistant professors, have more freedom, more time to work on the wards or in the laboratory, more leisure to think. The wise policy of establishing divisions within a department, and of giving division heads a large measure of autonomy, provides the sense of independence that most people need to have. It is difficult to understand, then, why these jobs shouldn't be so satisfactory that the men who hold them would be content with them for life, covet them as the remarkable opportunities they are. Most of them are now coming to offer tenure so that security is not involved. The assistant professor in a medical school often has an income which equals that of a department chairman in other branches of the University. In terms of academic standards, therefore, income is usually good although it obviously won't allow standards of living comparable to those of men in practice. Perhaps the desire to run things, to be "top dog" is so strong in the human race that the security, the opportunity and freedom to work within a Universify on problems of one's own choosing just won't provide to most people the measure of satisfaction they want from life. I am naive enough, however, to believe that if we would stop blowing up the professor as if he were some superhuman colossus, and would wave a flag more vigorously for the other jobs within a department, we might be able to focus on them as the prizes they are.

\section{II}

But if non-administrative departmental positions are to be kept so attractive that men will accept them as lifetime opportunities, both the departmental chairman and his men have a job to do. The former, confronted with increased teaching and service responsibilities himself, must not ask his associates to take over so many of these duties that he destroys their creative effectiveness. His task here is not an easy one. During the period of expansion most of us have recently been through, departments have been asked to take over the responsibility for more patients in their own university hospitals, in veteran institutions and in community hospitals which wanted to affiliate with the University. Any decision by a medical school that the saturation point has been reached is likely to prove unpopular. Most schools have yielded to pressure by increasing the size of their classes, often to the point of greatly reducing faculty-student ratios. The departmental chairman has to be most ingenious under these circumstances in order not to overload his men. In addition, he must give them all the autonomy possible, must make every effort to see that they get the recognition they deserve, and must fight to keep their incomes adequate.

But those of us who have never been chairman of a department often blame the chief of the service too much, and ourselves too little, when free time vanishes. The traps we fall into are fairly obvious, but most men never 
learn to avoid them: too many speaking engagements, too many conferences, too many committee meetings. One might follow that by saying: too much talk, too little work. It is reminiscent of the New Yorker story about a maid who quit her job because there was "too much switchin' of the dishes for the fewness of the food." Most men appreciate the recognition provided by these outside assignments, of course, and have a responsibility to accept their share, but the proper balance is hard to keep. Then there is the temptation to take oneself out of the academic income bracket by seeing more and more private patients or by assuming additional extraneous duties mostly because of the money involved. The price we have to pay in medicine, particularly in clinical departments, for the great privileges of an academic life, for the stimulating association with brilliant colleagues, for the opportunity to do just what we want to do, is so much less than that required of our other university associates that most salary protests have a hollow ring. Income bought at the expense of free time for creative work will certainly, for the investigator, seem like a poor investment when the age of 65 or 68 rolls around.

\section{III}

The danger also exists that each division head, when given a large measure of autonomy, will be tempted to build a little empire of his own without regard for the structure of the department as a whole. There is a disease which is fostered by this system. As departments increased in size, most medical schools had to use for salaries the money that formerly had supported research. Governmental and foundation research funds, fortunately, became more plentiful and filled the hole. Each man was told to apply for his own research grants. There then developed what may be called the "centrifuge-scintillation counter-Beckman disease." Formerly, one or two centrifuges would suffice for a whole department. Now, every laboratory must have at least one. This disease has characteristic symptoms and signs. There is acute depression if the laboratory budget is less than $\$ 100,000$ a year. There is unhappiness if there are so few fellows and technicians that the principal investigator has to do any work himself. No experiment involving radioactive isotopes can possibly be undertaken unless the laboratory has at least one counter from every instrument company in the field. There are more pieces of equipment than ideas. There is an amazing lack of any sense of responsibility for the wisest possible use of the funds. I think this preoccupation with equipment and large operations unfortunate, not only because of the waste involved, but because it tends to generate a false conception of research. It involves young men in the task of using instruments to pick up little pieces of information that have been passed over by more original investigators, encourages them to remain in laboratories past the time when they should have realized that their talents lay principally in other directions, or denies them the chance to explore more exciting leads because a longer time might be needed to obtain publishable results.

\section{IV}

This harangue was prompted only by : 1) the hope of convincing young men that the medical academic ladder is not as clogged as is the membership of this Soceity, 2) the desire to plea that non-administrative departmental jobs be organized to preserve the freedom necessary for active investigative programs, and 3 ) by the fear that unwise use of research funds may discourage the proverbial goose. Lastly, I would like to talk briefly about the meet-

\section{MEMBERSHIP DISTRIBUTION AMERICAN SOGIETY FOR CLINICAL INVESTIGATION 1954}

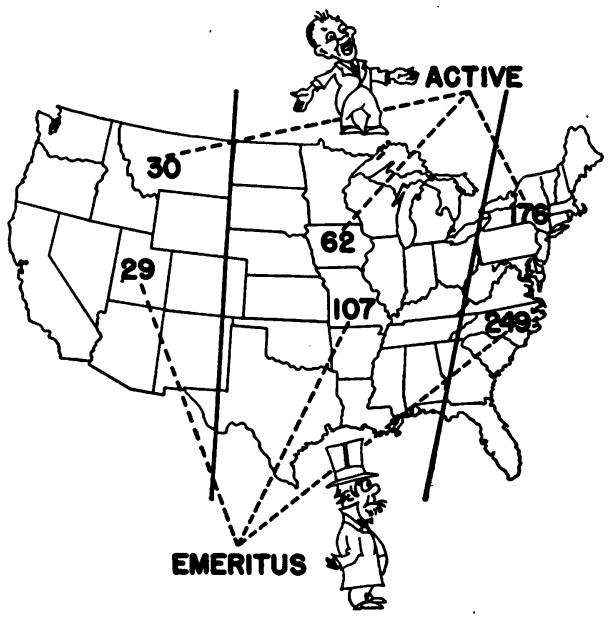

Figure 1

ing place of this Society. The meetings of the American Society for Clinical Investigation exert a tremendous influence on young men, and on clinical investigation as a whole in the United States. Ninety-two men or 34 per cent of our membership now come from the middle west or far west (Figure 1). House officers and fellows from these areas, year after year make the pilgrimage to Atlantic City at great expense to themselves or to their department, but only a fraction of them can make the trip. The time has come, it seems to me, for us to recognize the "American" in our name, to meet in other sections of the country as well as here. On purely democratic grounds there is no right at present to ask that more than one out of every three meetings be held in a central location. That, at least, should be done so that the splendid influence of these sessions can be spread over a broader section of the country. Men have discussed such a plan on the Boardwalk for years; councils have debated it ; the time is ripe for one of your presidents to recommend it from here. 


\section{ABSTRACTS}

Observations on the Capacity of Serum Alpha Globulin to Bind Thyroxin in Vitro. Edwin C. Albright, Frank G. Larson and William P. Deiss, Madison, Wis. (Introduced by Ovid O. Meyer).

Previous studies demonstrated the association of thyroxin with a specific serum alpha globulin in vivo. This specific alpha globulin binding was also demonstrated in vitro using $\mathrm{I}^{181}$ labelled thyroxin.

The thyroxin binding capacity of this protein was studied in hyperthyroid, euthyroid and hypothyroid subjects. Quantities of $\mathrm{I}^{181}$ labelled thyroxin were added to serum to yield concentrations of from .05 to 1.0 micrograms/milliliter. The thyroxin serum mixtures were subjected to paper zone electrophoresis at $\mathrm{pH} 8.6(0.1 \mathrm{M}$ veronal buffer). Following electrophoresis the distribution of radioactivity was determined.

In all sera the thyroxin appeared in the alpha globulin zone. At the lowest concentrations used (.05 to .1 micrograms/milliliter) only a very small amount of thyroxin appeared in the albumin. As the concentration of thyroxin was increased from .1 to $1.0 \mathrm{micrograms} / \mathrm{milliliter}$ a greater proportion of thyroxin appeared in the albumin as the alpha globulin binding capacity appeared to become relatively saturated. The concentration of thyroxin required to produce equal distribution of radioactivity between the alpha globulin and albumin zones has been used as a convenient measure of alpha globulin thyroxin binding capacity. This equilibration was found to occur with concentrations of .2 to $.5 \mathrm{micrograms} / \mathrm{milliliter}$ in the hyperthyroid sera, from .6 to $.8 \mathrm{micrograms} / \mathrm{milliliter}$ in the euthyroid and approximately $1 \mathrm{microgram} / \mathrm{milliliter}$ in the hypothyroid. Addition of iodide in vitro in concentrations from .001 to 10 micrograms/milliliter did not affect the thyroxin binding capacity. Administration of Lugol's solution to the hyperthyroid subjects appeared to increase thyroxin binding capacity somewhat.

It appears that in vitro thyroxin binding capacity of serum alpha globulin varies inversely with the state of thyroid gland activity. This is assumed to indicate that this protein in hyperthyroid subjects is relatively saturated with endogenous thyroxin, and conversely relatively unsaturated in the hypothyroid.

Water Content of Hyperlipemic Serum. Margaret J. Albrink, Pauline M. Hald and Evelyn B. Man, New Haven, Conn. (Introduced by C. N. H. Long).

The displacement of serum water by the lipids of lactescent serum was forcefully demonstrated in a patient whose low serum electrolyte values were directly attributable to the large volume occupied by the insoluble lipids of his. serum. The patient, an 18 year old boy with long-standing skin xanthomas, was admitted with mild diabetic acidosis and markedly lactescent serum.
His serum sodium was $103 \mathrm{mEq} . / \mathrm{L}$. and his serum chloride was $75 \mathrm{mEq}$./L., values quite out of keeping with the mildness of his illness. When the insoluble fat was removed from the serum by ultracentrifugation, the sodium content of the clear subnatant fluid was $135 \mathrm{mEq}$./L. and the chloride $100 \mathrm{mEq}$./L. The serum water determined gravimetrically was $73 \mathrm{gm} . / 100 \mathrm{ml}$. serum. The total fatty acids were $526 \mathrm{mEq} . / \mathrm{L}$., and the cholesterol 1219 mg. per cent.

An ensuing study of sodium, water, and lipid content of several lactescent sera revealed that as lipids increased there was a growing discrepancy between serum sodium and sodium recalculated in terms of serum water.

These findings prompted a search for a rapid and simple method for the estimation of serum water. A preliminary study indicates that a method involving the measurement of freezing-point depression of serum before and after addition of a known amount of dry sodium chloride meets this requirement. The determinations, requiring $2 \mathrm{ml}$. of serum, were accomplished by means of a freezing-point osmometer calibrated to give the osmolarity of the fluid analyzed.

Determinations were carried out on 10 different samples of biologic fluid, including several lactescent sera. The water content of these samples ranged from 78 per cent to 99 per cent. The values obtained osmotically agreed within one per cent with those obtained by the gravimetric method.

This method has the advantage of affording a direct estimation of serum osmolarity.

Elevated SPCA (Convertin) Complex in Pregnancy. Its Possible Role in Pathogenesis of Thromboembolism. Benjamin Alexander,* Lillian Meyers, Robert Goldstein, Victor Gurewich and Lester Grinspoon, Boston, Mass.

Although abnormal coagulation has been incriminated as a basic cause of thromboembolism, convincing evidence is lacking. Recent identification of new clotting factors raised the possibility that abnormalities in one or more of these substances might underly the development of thromboembolic disease. To explore this, the pregnant state was selected because it constitutes a common, temporary, reversible, relatively physiologic "deviation" which is frequently complicated by thromboembolism.

In a study of 57 pregnant women the plasma Spca complex was distinctly elevated, prothrombin less frequently and to a lesser extent. The Spca tended to increase further as term approached, and reverted toward normal within days after delivery. The average Spca of the gravid was $189 \%$ of normal (Owren method) compared with $82 \%$ for the non-pregnant group (41). In ten patients the Spca values were three to five fold the 
control average; in five additional patients the levels exceeded the non-pregnant average by more than five fold. In 24 subjects the average level three to five days postpartum was $114 \%$ contrasted with $150 \%$ shortly before delivery. In two individuals, striking Spca elevations persisted longer.

Prothrombin was less significantly altered. However, in nine women the prothrombin was between $140-180 \%$ of normal compared with an average level of $98 \%$ (range 58-128) in the controls.

The data support the concept of hypercoagulable blood in pregnancy. The role of Spca in accelerating thrombin evolution, its activation by thromboplastin, and its effectiveness in inducing intravascular thrombosis in animals (Wessler) are known. Excesses of this clotting factor, together with venous stasis and increased platelet adhesiveness already demonstrated in pregnancy $(H$. $P$. Wright), provide a delicately balanced stage which could readily be triggered to induce thrombosis by liberation into the circulation of thromboplastic material from the gravid uterus. The latter is known to occur.

Chronic Hypercapnia: A Specific Respiratory Depressant in Chronic Pulmonary Disease and Other Conditions. James K. Alexander, John A. Wood and John R. West, New York, N. Y. (Introduced by Dickinson W. Richards, Jr.).

That patients with pulmonary emphysema and chronic respiratory acidosis have a diminished ventilatory response to carbon dioxide inhalation seems well established. Possible mechanisms are (1) increased buffering capacity of the blood associated with (a) elevated plasma bicarbonate or (b) polycythemia; (2) failure of the chest bellows to respond normally to the central nervous stimuli; (3) diminution in the sensitivity of the nervous regulatory mechanism to the carbon dioxidehydrogen ion stimulus as a result of (a) congestive heart failure, (b) chronic acidosis, (c) chronic anoxemia or (d) chronic hypercapnia. Each of these possibilities has been investigated by relating the effective alveolar ventilation to the changes in arterial carbon dioxide tension and hydrogen ion concentration produced by addition of carbon dioxide to the inspired air.

Since the ventilatory response in these patients was reduced relative to the stimulus in terms of the hydrogen ion concentration as well as the carbon dioxide tension, increased buffering capacity of the blood did not account for the results. The diminished ventilatory response was independent of congestive heart failure and mechanical ventilatory impairment. Further observations on patients suffering from chronic anoxemia secondary to cyanotic congenital heart disease, or chronic metabolic acidosis associated with uremia, revealed no reduction in sensitivity to the carbon dioxide-hydrogen ion stimulus under these conditions. However, patients with chronic hypercapnia and an increased alkaline reserve associated with metabolic alkalosis were found to have substantial reductions in sensitivity to the carbon dioxide-hydrogen ion stimulus.
It is concluded that chronic hypercapnia per se results in a diminished sensitivity of the respiratory nervous regulatory mechanism to the carbon dioxide-hydrogen ion stimulus.

An Investigation of the Validity of "Insulin-resistance" in Diabetes Mellitus. Louis K. Alpert, Alvin E. PARRISh and KarL P. Kolb, Washington, D. C. (Introduced by Theodore J. Abernethy).

This study was undertaken to determine whether inhibition of insulin activity could be demonstrated in diabetic subjects.

A constant state of carbohydrate utilization was established by a continuous intravenous infusion of $240 \mathrm{mgm}$. of glucose per minute. When the blood glucose levels had stabilized, regular insulin, 5 units per $\mathbf{M}^{2}$, was injected intravenously. The rate of fall in blood glucose (mgm. per $100 \mathrm{cc}$. per minute) during the subsequent 45 minutes of continued infusion was used as the index of response to insulin.

49 diabetic and 31 non-diabetic patients were studied. In the non-diabetics, the mean index was 1.06 (S. D. \pm 0.36 ), and in the diabetics was 1.51 (S. D. \pm 1.28 ). The individual indices in the non-diabetics ranged from 0 to 2.0 , with $85 \%$ falling between 0.5 and 1.5 . In the diabetics the range was 0 to 6.5 , with $48 \%$ falling between 0.5 and 1.5 , and $35 \%$ between 1.5 and 2.5 . The values of the indices were unrelated to the blood sugar levels of the patients, or to the daily requirements for insulin.

It is concluded that these diabetic patients, with the method employed, appeared to show an equal or greater response to insulin than did the non-diabetic individuals. These observations, if valid, would be compatible with the concept that a) diabetes mellitus may be due essentially to an endogenous lack of insulin production, and b) variations in insulin requirements may be associated with independent gluconeogenetic factors.

Exchange of Cations, Respiratory Gases and Carbohydrate by Intact Human Muscle. R. ANDRES, K. L. Zierler,* G. Cader, A. S. Ghrayyib and J. L. LilienTHAL, JR.,* Baltimore, Md.

As a first step in examining metabolic events in skeletal muscle in man, serial measurements in six subjects of respiratory gas exchange and movement of certain carbohydrates and cations have been made in the human forearm. Net exchange of metabolites was determined as the product of arterio-venous concentration difference and blood flow. The latter was determined by constant injection of T-1824 into the brachial artery and continuous sampling of venous outflow from a catheter placed deep in forearm muscles. This method demonstrated reasonably constant flow under "resting" laboratory conditions and a brisk response to exercise of forearm musculature.

In some subjects oxygen consumption and carbon dioxide production and their exchange ratios were relatively steady. In others random minute-to-minute variations were of extraordinary magnitude and were out of 
phase. For the entire group, oxygen uptake per forearm ranged from 0.9 to $5.1 \mathrm{ml} . / \mathrm{min}$., and carbon dioxide production from 0.3 to $6.3 \mathrm{ml} . / \mathrm{min}$., with exchange ratios varying from 0.12 to 2.05 .

Traditionally, in vitro studies have indicated that the major part of oxygen utilized by resting muscle is consumed in oxidation of glucose. Anaerobic degradation of glucose is accounted for largely by the appearance of lactate. However, in these studies such a balance was demonstrated in only a few instances. In the majority of cases none or little of the oxygen used could be accounted for by aerobic glucose utilization.

Potassium appeared to leave the forearm in small quantities during "rest", and the rate of escape was accelerated by exercise. Net exchanges of sodium were random in direction.

These studies, designed to provide basic data for subsequent assessment of altered muscle metabolism, indicate remarkable minute-to-minute instability of metabolic exchanges in "resting" muscle and suggest that carbohydrate metabolism does not always account for the major fraction of oxygen consumed.

A Comparison of Dextran and PVP in Human Volunteers. John B. Armstrong, Winnipeg, Canada. (Introduced by J. Doupe).

Five hundred or $1000 \mathrm{ml}$. quantities of $6 \%$ British or Swedish dextran were administered to 12 normal subjects, and of $3.5 \%$ polyvinylpyrrolidone to 12 . Serum and urine determinations, together with haematocrit and plasma protein concentrations were carried out before, immediately after, and at 24 hour intervals following infusion.

The serum concentration fell more sharply with Swedish than with British dextran. PVP showed a more rapid early decline in serum concentration but was better maintained after 24 hours. These changes were reflected in the urinary excretion. Both dextran and PVP caused a fall in haematocrit and plasma protein concentration immediately following the infusion, which disappeared as their blood levels fell.

Seven dextran subjects received a prior venesection, and showed a haematocrit dilution persisting well beyond that found with dextran alone. To elucidate, 10 subjects underwent venesection only. They showed a delayed dilution of the haematocrit suggesting that the persistent dilution was due to the early effects of dextran and the later effects of venesection. The plasma protein dilution following venesection and dextran was not different from dextran alone. This may be explained by the observed rapid disappearance of plasma protein dilution following venesection only.

Seven subjects received a venesection followed by 500 ml. isotonic saline. The haematocrit and plasma protein dilution was not significantly different from venesection only. This indicates a rapid loss of saline from the circulation in contrast to the slower loss of dextran and PVP.
The discordant shifts of haematocrit and plasma protein dilution suggest that neither is a reliable index of plasma volume changes following haemorrhage.

Adrenal Cortical Activity in Normal Men on Diets of Low Sodium Content. B. J. Axelrad and J. A. Luetscher, JR.,* San Francisco, Calif.

When dietary sodium intake of normal men was reduced from 150 to $15 \mathrm{mEq}$. per day, urine sodium fell abruptly to low levels by the third day and reached a minimum by the fifth day of the low sodium diet. Insignificant changes in daily creatinine excretion and in inulin clearance indicated that tubular reabsorption of sodium increased during this period of low sodium intake.

Previous work on edematous patients showed that chloroform extracts of urine acidified to $\mathrm{pH} 1.0$ contained high sodium-retaining activity demonstrable by bioassay. Sodium-retaining activity could be demonstrated in normal men on unrestricted sodium intake only if the urine was subjected to prolonged acid hydrolysis, and if large dosages of extract were assayed. Measured under these conditions, the normal control output of activity was increased five fold by the third day on low sodium diet, and remained at this level until normal sodium intake was resumed on the 7 th day.

The material responsible for the sodium-retaining activity had the chromatographic mobility characteristic of the sodium-retaining corticoid isolated from the urine of edematous patients with heart failure or nephrosis and of "electrocortin" from adrenal cortical extract.

The increased output of sodium-retaining hormone was not accompanied by any significant change in output of 17-ketosteroids or 17-hydroxycorticoids measured by Forsham and Island. Diets of low sodium content appear to stimulate primarily the sodium retaining hormone produced by the adrenal cortex.

The Use of Monocalcium Disodium Ethylene Diamine Tetra-acetic Acetate (CAEDTA) in the Treatment of Adult Lead Intoxication. WILLIAM H. BAKER, Boston, Mass. (Introduced by Joseph C. Aub).

Recently a new chelating agent, monocalcium disodium ethylene diamine tetra-acetate (CAEDTA), has been introduced for clinical trial in heavy metal poisoning.

CAEDTA has a strong affinity for lead and exchanges its calcium for lead to form a PbEDTA complex which is then excreted unchanged via the urine. We have administered this compound intravenously in a dosage of $\mathbf{0 . 5}$ gm. per 30 pounds of body weight to six patients with chronic lead poisoning and to three normal control patients. All six patients with chronic plumbism prior to treatment showed increased urinary lead and coproporphyrin levels. All patients had anemia with stippling of the red cells and abnormal fragility tests. CAEDTA was administered to each patient on consecutive days for periods of four to eighteen days. On the first day of administration there was a marked increase in urinary lead excretion and a simultaneous lowering of coproporphyrins. On succeeding administrations there was a gradual 
decrease in the amount of lead excreted while urinary coproporphyrins remained at normal levels. During the period of therapy all patients exhibited a rise in hemoglobin, stippling disappeared, and fragility tests returned to normal. In the three normal control subjects CAEDTA was administered on only one day with an increase in urinary lead on that day and no change in the coproporphyrin. Metabolic balance studies of electrolytes were performed on two patients with chronic lead poisoning. No alterations in metabolic balance occurred during administration of this compound either in a six or eighteen day study period.

These studies indicate that CAEDTA is an effective way of mobilizing deposited lead with simultaneous improvement in the patient's condition and no evidence of toxicity.

Urinary Concentrating Process during Antidiuresis in Renal Disease. David S. Baldwin, Harold J. Berman, and Henry O. Heinemann, New York, N. Y. (Introduced by Herbert Chasis).

Fixation of urinary specific gravity in renal disease is generally assigned to loss of renal tubular concentrating ability. In this study the urinary concentrating process has been examined by the cryoscopic method, and a tubular mechanism for the production of a hypertonic urine has been shown to persist in the course of various renal diseases.

Observations of glomerular filtration rate (GFR) and osmolarity of plasma and urine were made in the hydropenic state in 20 patients during Pitressin administration and with osmotic diuresis induced by mannitol. The quantity of water abstracted from the isosmotic glomerular filtrate in the formation of osmotically concentrated urine was calculated as the difference between the osmolar clearance and the urine flow and designed as $\mathrm{T}_{\mathrm{H}_{2} \mathrm{O}}$.

In each case a relatively constant $T_{\mathrm{H}_{2} \mathrm{O}}^{\circ}$ was attained, ranging in these patients from 0.8 to $8.2 \mathrm{ml}$. per min., while the range of GFR was from 12 to $96 \mathrm{ml}$. per min. In general, there were proportionate reductions in $\mathrm{T}_{\mathrm{H}_{3} \mathrm{O}}^{\mathbf{O}}$ and GFR with an average $T_{\mathrm{H}_{2} \mathrm{O}}^{\mathrm{c}} / \mathrm{GFR}$ ratio of 0.056 as compared to the normal ratio of $0.051 \pm 0.015$. However, in 2 patients recovering from acute renal failure there was a disproportionate impairment of $\mathrm{T}_{\mathrm{H}_{2} \mathrm{O}}^{\circ}$ as manifested by $\mathrm{T}_{\mathrm{H}_{2} \mathrm{O}}^{\mathrm{o}} / \mathrm{GFR}$ ratios of 0.017 and 0.008 , while in 2 acute nephritics the ratios were 0.086 and 0.072 .

Lack of correlation between urinary osmolar concentration and urine specific gravity as determined by the urinometer was demonstrated repeatedly. In no case did urine osmolarity fail to exceed plasma osmolarity, even in the presence of "fixed" specific gravity.

It is concluded that a maximal tubular reabsorptive rate for free water in the formation of a hypertonic urine is demonstrable in renal disease. With few exceptions this process deteriorates in proportion to the diminution of glomerular filtration, suggesting loss of functioning units rather than specific tubular dysfunction.
Radioactive Iodine for Chronic Lung Disease. Bernard A. Bercu and Harvey N. Mandell, St. Louis, Mo. (Introduced by Robert J. Glaser).

The present treatment for chronic pulmonary insufficiency is usually disappointing and frequently of only temporary benefit. Since one fundamental defect in chronic lung disease is a deficiency in the supply of oxygen to the body, it was thought worthwhile to determine the effect of lowering the basal requirements of the tissues for oxygen by making patients with chronic lung disease relatively hypothyroid.

Ten patients with pulmonary emphysema and/or fibrosis were selected. These patients had been treated repeatedly by the usual methods and were still markedly incapacitated either at rest or upon slight exertion. Since antithyroid treatment could not be expected to alter the fundamental pulmonary lesion, objective changes in lung function were not anticipated. In evaluating the effect of therapy, reliance was therefore placed upon standard exercise tolerance tests and upon subjective changes. Patients were considered significantly improved if they were able to perform activities which previously were intolerable because of dyspnea. Those who did not give evidence of increased exercise tolerance, even though they were improved subjectively, were considered as only questionably improved.

Of the ten patients treated, three were initially made hypothyroid with Tapazole. The other seven were given $I^{12}$ on one or more occasions. All patients were euthyroid prior to therapy. Seven patients were found to be significantly improved, one was questionably benefitted, and two were unchanged. In two patients severe dyspnea developed after the Tapazole was discontinued in preparation for the use of $\mathrm{I}^{12}$. This dyspnea again disappeared in one patient after establishment of hypothyroidism. The other patient expired during the interval.

\section{Influence of Age and Size of Bacterial Population on the Rapid Bactericidal Action of Penicillin. CArr Bernt- SEN, New York, N. Y. (Introduced by Ralph Tomp- sett).}

Relapse of infections caused by penicillin susceptible bacteria following penicillin therapy is an apparent inconsistency with its rapid bactericidal action on these same bacteria in vitro. The commonest explanation of this seeming paradox has been based on the intervention of an inflammatory lesion, with presumed failure of its penetration by metabolites or drug. These hypotheses have little experimental support.

In preliminary studies it was found that the total population of bacteria in lesions of experimental animals and man were higher than those customarily tested in vitro. Moreover, it appeared that the ages of individual members in such populations varied considerably. Accordingly, an investigation was made of the influence of age and size of bacterial populations on the bactericidal action of penicillin.

The test microorganism employed was a highly penicillin susceptible strain of Group A Streptococcus hemo- 
lyticus. Observations were made on young and old bacterial populations in both small and large inocula during prolonged exposure to penicillin in vitro and in the thigh muscles of mice.

The evidence obtained supports the hypothesis that the rapid bactericidal action usually ascribed to penicillin is a phenomenon customarily associated with small numbers of young bacteria tested in circumstances favorable to rapid growth. With increasing age of the population irrespective of the presence or absence of an inflammatory lesion, the rapid bactericidal action of penicillin was conspicuously diminished. No evidence was obtained that the presence of a lesion materially altered the phenomenon of the relative penicillin insusceptibility of bacterial populations exceeding twelve hours in age. Increases in size of bacterial populations of constant age did not change penicillin susceptibility.

It appears therefore that the rapid bactericidal action of penicillin is an unnatural phenomenon which depends upon conditions of infection not to be anticipated in human infections.

The Behavior of the Formed Elements of the Blood During Leukocyte Infusion and Cross-Transfusion in Man. Howard R. Bierman, * Keith H. Kelly, Ralph L. Byron, Jr., and Fauno L. Cordes, Duarte, Calif.

By temporarily depleting the circulation of leukocytes to a stable level, the return of the leukocytes into the circulation might afford an estimate of leukocyte production and delivery. Exploratory studies to determine the feasibility of various methods for leukocyte depletion were undertaken. Arterial cross-circulation and replacement transfusion were employed to determine the rate of disappearance, removal and delivery of leukocytes in man. Eleven cross-transfusions in nineteen patients with various neoplastic diseases, including the leukemias, were completed. Determinations of the total and differential leukocyte counts during the cross-transfusion and immediately thereafter demonstrated the disappearance and return of the various forms of leukocytes in the peripheral blood in both the donor and recipient. Serial bone marrow aspirations afforded additional information.

The pulmonary circulation in non-leukemic man was shown to be capable of removing large numbers of infused leukocytes. The erythrocyte, hemoglobin, and hematocrit determinations followed the simple laws of mixing. In general, an increase in platelet number was observed in both recipient and donor. The highest rate of leukocyte delivery and production was 59.6 million per minute and occurred in a patient with metastatic melanosarcoma. The lowest rate of leukocyte delivery and production was 1.3 million per minute in a child with lymphocytic leukemia.

In the cross-transfusion between two leukemic participants the leukocytes circulated freely in both patients. The disappearance rate of the various cell types after the cross-circulation could be described by the relationship; $N=c e^{-a t}+b$. The intravascular life span of the polymorphonuclear leukocyte in this study was esti- mated at 96 hours and that of the lymphocyte at 56 hours under the conditions of the study. The remaining types of immature granulocytes had intravascular life spans between 56 and 96 hours.

The clinical and other hematological findings will be presented.

Supported in part by National Cancer Institute, U. S. Public Health Service.

Comparative Effectiveness of Daily Doses of l-Triiodothyronine or l-Thyroxine in the Control of Myxedema. Charles M. Blackburn and F. Raymond Keating, $\mathrm{J}_{\mathrm{R} . \text {, }}$ Rochester, Minn.

Daily oral doses of 1-triiodothyronine and 1-thyroxine were compared on the basis of ability to maintain a euthyroid state and a normal metabolic rate over an extended period of time in patients who had myxedema. Among 10 such patients, maintenance doses of 1-triiodothyronine were found to vary from 52 to 105 micrograms.

Among 4 such patients, daily maintenance doses of 1-thyroxine were found to vary from 175 to 400 micrograms. Compared in this way, 1-triiodothyronine appeared to be about three to four times more potent than 1-thyroxine.

On the same basis, the oral versus the intravenous administration of 1-triiodothyronine was compared among 5 patients and the oral administration versus the intravenous administration of 1-thyroxine was compared among 3 patients. The agent, 1-triiodothyronine, appeared to be slightly more effective when used intravenously than when used orally, whereas 1-thyroxine was approximately twice as effective when used intravenously as when used orally. For example, 125 micrograms of 1-thyroxine given intravenously daily maintained a patient at the same metabolic level as that produced by 250 micrograms daily given orally. The intravenous administration of 1-triiodothyronine appeared two to three times as effective as the intravenous administration of 1-thyroxine.

Observations on Impaired Intrapulmonary Gas Mixing. EmII. BlaIR, Durham, N. C. (Introduced by Albert Heyman).

Uneven mixing of gas within the lung is common in pulmonary disease. A means of measuring and expressing this defect would be useful in estimating the severity of disease and the changes induced by therapy. The concept that an unequally ventilated lung functions as a number of smaller compartments, each evenly ventilated at its own rate, provides an approach to this measurement. This report presents a new method for measuring intrapulmonary mixing based on this concept. Pathologic abnormalities in mixing are described, and the influence of therapy demonstrated.

After breathing a $50 \%$ helium-50\% oxygen mixture for 15 minutes, the subject is switched to oxygen, and the expired air is continuously analyzed for helium by katharometer. From the helium washout curve, which reflects intrapulmonary mixing, and from the minute vol- 
ume of ventilation, it is possible to calculate size and ventilation rate of the most poorly aerated lung compartments. The functional residual capacity (FRC) can be calculated from the total helium excretion.

By this method about one-half of normal subjects show relatively slow ventilation in a lung compartment comprising $10-50 \%$ of the FRC. Intravenous aminophyllin usually clears this impairment almost completely in normal subjects. Patients with emphysema show gross mixing defects, to the extent that two-thirds of the FRC may receive only a tenth of the ventilation volume. In ten patients with asthma, or asthma and emphysema, aminophyllin produced marked improvement in ventilation of "slow spaces" and decrease in FRC. In ten cases of emphysema without evident bronchospasm aminophyllin often had the paradoxical and unexpected effect of increasing the size of the worst ventilated space and enlarging the FRC.

The method appears useful in quantitating impairment of intrapulmonary mixing and in following changes resulting from progress of disease or from therapy. It also provides further insight into the mechanisms of pulmonary dysfunction.

The Effect of Oral Feedings on Splanchnic Blood Flow and Oxygen Consumption in Normals and in Subjects with Hepatic Cirrhosis. J. Leonard Brandt, Leonard Castleman, Jack Greenwald and J. J. Kelly, JR., Brooklyn, N. Y. (Introduced by William Dock).

The hemodynamic and metabolic effects of oral protein in seven normal and five patients with Laennec's cirrhosis were determined utilizing the hepatic vein catheterizationBSP clearance technique. Similar studies were made in three normal and two cirrhotics following oral glucose.

For the group fed a protein meal (250 grams homogenized lean beef), fasting estimated splanchnic,blood flow (ESBF) averaged $1160 \mathrm{cc} / \mathrm{min} / \mathrm{m}^{2}$ in normals, and 795 $\mathrm{cc} / \mathrm{min} / \mathrm{m}^{2}$ in cirrhotics. Fasting splanchnic consumption of oxygen (SOC) averaged $45.8 \mathrm{cc} / \mathrm{min} / \mathrm{m}^{2}$ in normals and $43.9 \mathrm{cc} / \mathrm{min} / \mathrm{m}^{2}$ in cirrhotics. The similarity in fasting SOC between the two groups is accounted for by an arterial-hepatic vein (A-HV) oxygen difference of 4.2 vol.\% in normals and 5.2 vol.\% in cirrhotics. During 120 minutes of observation post prandially, average maximal increase in ESBF over controls was 33\% in normals and $27 \%$ in cirrhotics; average maximal increase in SOC was $55 \%$ in normals and $14 \%$ in cirrhotics. During the first hour post prandial, average A-HV oxygen difference rose 0.5 vol. $\%$ above controls in normals, and fell 0.5 vol.\% below controls in cirrhotics. During the second hour, average A-HV oxygen difference was 0.6 vol.\% above controls in normals, and 0.3 vol.\% above controls in cirrhotics.

In three normal subjects and two cirrhotics fed a glucose meal (100 grams glucose) there was a consistent fall below fasting levels for both ESBF and SOC of approximately $10 \%$ in both measurements. In no instance did either measurement show an increase after oral glucose feeding.
Thus, quantitative but not qualitative differences in the rises of ESBF and SOC were determined in both normals and cirrhotics following an oral protein feeding. Following a glucose feeding consistent falls in ESBF and SOC were demonstrated. The possible clinical implications of these observations will be discussed.

Blood Volume in Congestive Heart Failure Studied by Cr, CO, and T-1824. Methods. Ellen Brown, James Hopper, Jr., Norman Nomof, Reidar Wennesland and Kenneth G. Scott, San Francisco, Calif. (Introduced by P. H. Forsham).

Three methods were used to estimate blood volume in 25 patients with severe congestive failure, none with primary pulmonary disease: two RBC-tagging methods (CO with 20-minute sampling and $\mathrm{Cr}^{\text {sit }}$ with serial sampling), and the 10-minute T-1824 method. The 3 methods were applied simultaneously in 10 experiments. Whole blood volume (VWB), in $\mathrm{ml} / \mathrm{Kg}$, was $20 \%$ or more above mean predicted normal for the method in 9 of 13 patients studied with $\mathrm{Cr}^{\text {s2 }}$, and in 15 of 20 studied with CO. RBC volume (VRBC) was also increased more than $20 \%$ above predicted in the majority of experiments with celltagging methods. With $\mathrm{Cr}^{51}$, VWB ranged between +8 and $+64 \%$, and VRBC between zero and $+50 \%$ of normal. The apparent $\mathrm{T}-1824$ space was between zero and $+62 \%$ of predicted, and VWB by dye and hematocrit between zero and $+44 \%$. However, analysis of the mixing curves of $\mathrm{Cr}^{\mathrm{si}}$ which were obtained simultaneously showed that mixing had been incomplete at 10 minutes in several experiments, so that larger $T-1824$ spaces would have been found if sampling had occurred later. For the 6 experiments where mixing was complete at 10 minutes, body hematocrit was calculated as $\mathrm{Cr}^{51}$ space $\times$ hematocrit

$\overline{\mathrm{Cr}^{51} \text { space } X \text { hemat. }+\mathrm{T}-1824 \text { space. }}$ Hematocrits were corrected for plasma trapping using Chaplin's data (Blood, Vol. 7, p. 1227, 1952). Body hematocrit was normal or high ( 89.2 to $99.2 \%$ of venous hematocrit) in 5 instances; it was as low as $82.4 \%$ only once. Nine patients were followed by serial measurements ( 2 with $\mathrm{Cr}^{81} ; 7$ with CO). Coincident with clinical improvement, VWB fell ly 470 to $1920 \mathrm{ml}$ in 8 cases, and VRBC decreased by 180 to $600 \mathrm{ml}$ in 7. It is concluded that VWB and VRBC frequently are increased in congestive failure, and decrease with improvement. The relationship between "body" and venous hematocrits probably does not invalidate use of cell-tagging methods for blood volume estimation in this condition.

Corticosteroid Metabolism in Liver Disease. HaroLd Brown and Leo T. SAMuels, Salt Lake City, Utah. (Introduced by B. V. Jager).

The development of techniques for the measurement of the 17-hydroxycorticosteroid levels in plasma and urine has made possible a study of the metabolism of these compounds in normal subjects and in patients with liver disease. 
Plasma levels of 17 -hydroxycorticosteroids were normal in the patients with liver disease. After a standard infusion of hydrocortisone in twelve patients with liver disease and in eleven normal subjects, the 17-hydroxycorticosteroids disappeared from the plasma in a logarithmic fashion, at a rate which was proportional to the functional capacity of the liver as measured by the degree of BSP retention. In patients with hepatitis, serial studies demonstrated an increased rate of 17 -hydroxycorticosteroid removal as the liver function improved.

As compared with hydrocortisone, tetrahydrocortisone, which has been shown to be the major 17-hydroxycorticosteroid excreted in the urine, disappeared from the plasma at a much more rapid rate which was independent of liver function.

The rise in the plasma 17-hydroxycorticosteroid levels in response to ACTH stimulation in the patients with liver disease was within normal limits.

The daily urinary excretion of 17 -hydroxycorticosteroids of the patients with liver disease was $3.5 \pm 0.4 \mathrm{mg}$. as compared with $5.5 \pm 1.2 \mathrm{mg}$. for the normal group. These results differ from those of other workers who used less specific methods for the measurement of adrenalcortical steroids.

These data indicate that the removal of 17-hydroxycorticosteroids from the plasma is dependent upon the functional state of the liver. Hence the plasma level of these steroids depends at least in part on the functional state of the liver as well as the rate of adrenalcortical secretion.

The Determination of Pressure Gradients in the Vascular System of Man. Robert A. Bruce,* CURT A. WIEDERhielm, James J. Pampush and Henry J. Kowalski, Seattle, Wash.

Recording of instantaneous pressure gradients between any two points in the vascular system previously used in animal studies has been simplified and applied to humans during cardiac catheterization.

Direct pressures from two sites, the instantaneous difference between these sites and the electrocardiogram can be simultaneously recorded on a Sanborn Poly-viso. A double lumen catheter, whose orifices are $10 \mathrm{~cm}$. apart, is used with Statham strain gages. Instantaneous pressure differences are derived by electrical subtraction of one impulse from the other and inscribed directly as a gradient in $\mathrm{mm}$. Hg. Electrical calibration can be done repeatedly. Gradients of $0.5 \mathrm{~mm}$. $\mathrm{Hg}$ are readily determined. Limiting factors appear to be the physical properties of the catheters insofar as they contribute to resonance and damping by their distensibility characteristics.

The pressure gradient between inferior vena cava and right auricle may reverse during each heart cycle. Flow here may be discontinuous or phasic.

Pressure gradients across tricuspid and pulmonic valves and through intracardiac defects can be obtained. Premature ventricular systoles markedly alter the gradient between right ventricle and right auricle and indicate in- complete closure of the valve during the abnormal contraction.

Respiratory variations induced by breathing, coughing, and the Muller and Valsalva maneuvers affect pulmonary arterial and "capillary" pressures, but have little effect on the gradient within the pulmonary artery. Since arterial-"capillary" gradients may be altered by changes in intrathoracic pressure, pulsatile flow through the capillary bed is modified by respiration. The effects of mitral valve disease on PA-PC pressure gradients can be observed.

It is concluded from these preliminary observations that simultaneous differential pressure measurements during cardiac catheterization are simple, reliable and valuable adjuncts to the studies usually made. The technique is applicable to the instantaneous differentiation of any phenomena that can be recorded electrically with this equipment.

Synergistic Effect of Polymyxin B and Oxytetracycline or Tetracycline Upon Growth of Pseudomonas Aeruginosa. Paul Bunn,* Leonard Canarile and Winifred OsBORNE, Syracuse, N. Y.

Forty-three strains of Pseudomonas aeruginosa derived from human infections were tested for sensitivity to eight antimicrobial agents. Maximal inhibiting concentrations were determined by the serial dilution technic, using a $10^{-5}$ dilution of organism as the inoculum. All strains were resistant to more than 6.0 micrograms per $\mathrm{ml}$. of penicillin, streptomycin, chlortetracycline, tetracycline, oxytetracycline, chloramphenicol and bacitracin but were sensitive to less than 3.5 micrograms polymyxin B per $\mathrm{ml}$. The maximal inhibiting concentration of polymyxin $B$ failed to control growth of all strains after four hours when an undiluted culture was used as the inoculum.

Complete inhibition of growth of the undiluted culture of pseudomonas was achieved for more than eight hours when either one-quarter the sensitivity level of polymyxin was combined with the maximal inhibiting concentration of oxytetracycline or tetracycline, or when one-half of those concentrations was employed. This effect was uniformly greater than that observed with double the maximal inhibiting levels of the three agents singly, both in duration of effect and in number of bacteria killed.

This synergistic effect upon inhibition of growth of Pseudomonas aeruginosa could not be demonstrated with four other combinations of antimicrobial agents.

Sterilization of the urine of four humans with chronic Pseudomonas aeruginosa urinary trace infections was accomplished with a dosage regimen of 25 milligrams polymyxin B once daily parenterally combined with 2 grams orally of either tetracycline or oxytetracycline. In a fifth case a few colonies of pseudomonas persisted in the urine after seven days but their ability to produce green pigment was lost. Toxicity to this regimen was not observed and no strain of Pseudomonas aeruginosa became resistant to the three agents during the period of study. 
The Tissue Utilization of Endogenous Thyroid Hormone in Human Subjects. Belton A. Burrows, Theodore Peters and Joseph F. Ross,* Boston, Mass.

We have estimated the peripheral utilization of endogenously produced thyroid hormone in human subjects from the amounts of iodide being released from the catabolized hormone. The values depend on the size of the extrathyroidal iodide pool, and the rate at which it is being turned over.

Such studies are possible if added iodide equilibrates rapidly with the iodide pool in comparison to the turnover rate. In five myxedematous patients, in whom equilibration might be slow, the radioiodide spaces one hour after an intravenous tracer dose of I-131 averaged 1.5 times the simultaneously determined radiosulfate spaces, taken as an index of extracellular fluid volume, and approached maximum values. This indicates rapid equilibration of iodide returning from the tissues with the iodide pool.

The turnover rate of the iodide pool was indicated by the rate of decrease of its specific activity (I-131/I-127), due to the release of I-127 from catabolized thyroid hormone in the tissues. It averaged 22.4 percent per hour in the normal subjects as compared with 37.9 percent per hour in the hyperthyroid individuals. The iodide pool was virtually constant in individual patients during the period of study, averaging 48 micrograms of I-127 in the normal group. The average value for the hyperthyroid group was considerably higher, 295 micrograms of I-127, due mainly to an increased serum concentration of inorganic I-127.

The estimated amounts of hormonal iodide released from the tissues in the normal group ( 8.5 micrograms per hour) would be equivalent to the daily utilization of 300 micrograms of levo-thyroxine. In the hyperthyroid group the average peripheral iodide release was ten times greater (73.4 micrograms per hour), which would be sufficient to account for the observed hypermetabolism of hyperthyroidism.

Ferrokinetics in Anemia of Infection. J. A. BusB and G. E. Cartwright,* Salt Lake City, Utah.

Ferrokinetic studies of $\left(\mathrm{Fe}^{\infty}\right)$ have been made in five patients with anemia associated with chronic infection and in seven normal subjects. Ten to $20 \mu \mathrm{C}$ of $\mathrm{Fe}^{\mathrm{s}}$ containing less than $6 \mu \mathrm{g}$ of total iron were incubated with the individual's own plasma, and the labelled plasma was injected intravenously. Blood volumes were determined by means

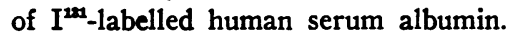

In none of the patients was clinical jaundice present, and there was no detectable increase in urobilinogen excretion in the stools. The mean volume of packed red cells in these patients was $37 \mathrm{ml} . / 100 \mathrm{ml}$., the plasma iron $38 \mu \mathrm{g}$ per cent, and the reticulocytes 1.4 per cent.

In the patients with infection the half-time of plasma $\mathrm{Fe}^{\mathrm{\omega}}$ disappearance was 0.50 hours; the plasma iron turnover rate was $0.64 \mathrm{mg} . / \mathrm{kg} . / 24$ hours; the red blood cell iron turnover rate was $0.62 \mathrm{mg} . / \mathrm{kg} . / 24$ hours; the red cell iron renewal rate was 1.9 per cent/ 24 hours. In the normal subjects the half-time of plasma $\mathrm{Fe}^{60}$ disappearance was 1.43 hours; plasma iron turnover rate, 0.52 mg./kg./24 hours; red cell iron turnover rate, $0.48 \mathrm{mg} . /$ kg./24 hours; red cell iron renewal rate, 1.2 per cent/24 hours.

Incorporation of radio iron into the erythrocytes occurred somewhat more rapidly in the patients with infection as compared with the normal individuals. The per cent of the administered dose which ultimately appeared in the erythrocytes was the same in each group.

The above data suggest that the erythrocyte survival time of patients with chronic infections is slightly shortened ( 60 per cent of the normal), and that erythrocyte production although slightly increased above the normal (approximately 1.3 times) is not increased sufficiently to prevent the development of a mild degree of anemia. When consideration is given to the fact that a normal marrow is capable of increasing its production rate 6 to 8 fold, it is apparent that there is a marked inhibition of erythropoiesis in such patients.

\section{A Study of the Electrical Activity of Intact and Par-} tially Mobilized Human Ureters. HARvey R. BuTCHER, JR. and William Sleator, JR., St. Louis, Mo. (Introduced by Carl A. Moyer).

Little is known regarding the part played by abnormalities of ureteral activity in the genesis of ills of the upper urogenital tract. Acute hydroureter and hydronephrosis have become increasingly important medical problems as uretero-intestinal anastomosis has become one of the common surgical procedures.

A method of intraureteral electroureterography has been developed in order to investigate the effect of an uretero-intestinal anastomosis upon the activity of the ureter. The method entails bipolar recording of ureteral peristaltic action potentials from intraluminal electrodes. After performing extensive preliminary investigations upon the anesthetized dog, the method was applied to the study of ureteral activity in man before and after surgical manipulation of the ureter.

The investigation of normal human ureteral activity has led to a number of new observations:

(1) The shape of the recorded ureteral peristaltic action potential in man is similar to that of the dog. Control experiments indicate that the voltage-time contour recorded by this method represents actual changes in ureteral smooth muscle membrane potential. There is a "shoulder" during electrical recovery similar to that of action potentials recorded intracellularly from cardiac muscle.

(2) A large slow voltage change accompanies the passage of urine by the electrodes.

(3) Variations in the velocity of peristaltic waves correlate with changes in the shape of the recorded action potentials and with changes in the length of the interperistaltic wave interval.

The disturbance in ureteral function attending ureterointestinal anastomosis is a failure of propagation of the 
peristaltic wave through surgically disturbed segments of the ureter. Maximum duration of this disturbance in conduction is as yet unknown; however, it lasts at least the two to three days the electrode assembly has been left in the ureter postoperatively. Frequency and velocity of propagation of ureteral peristalsis in the undisturbed section of the ureter were unaltered by uretero-intestinal anastomosis.

Effect of Maintenance Cortisone Therapy on the Metabolic Response to Surgery. Evan Calkins and Donald M. Mrtchell, Boston, Mass. (Introduced by Walter Bauer).

Requirements for the normal response to surgery are not entirely understood. An opportunity for study was provided by the successive development of bilateral inguinal herniae in a patient with rheumatoid arthritis maintained on 75-100 mg. oral cortisone. On two occasions, under general and local anesthesia, respectively, he underwent herniorrhaphy while on the metabolic ward. Cortisone dosage was unchanged throughout each experiment. Diet was unchanged except that he was unable to eat on the day of ether anesthesia. The metabolic response was compared with that caused by subsequent sudden increase in cortisone to $250 \mathrm{mg}$. per day, and with balances which had been obtained on this patient prior to and immediately following initiation of cortisone therapy two years previously.

Data showed that surgery was followed by negligible change in nitrogen, potassium, calcium, and phosphorus balances and in creatine excretion. There was, however, striking sodium retention. A similar metabolic pattern accompanied onset of steroid therapy and sudden increase in dosage. The first operation was followed by a sudden fall in 17-ketosteroid excretion from 11 to $4.6 \mathrm{mg}$. and in formaldehydogenic steroid excretion from 36 to $16 \mathrm{mg}$. per day. Similar determinations were performed at the time of the second operation.

These results indicate that this patient (presumably because of his depletion) reacts to the stress of surgery and to increase in cortisone primarily with sodium retention, rather than with an acute catabolic response as seen in normal individuals. The fact that he displayed this characteristic response following surgery, despite suppression of adrenal cortical activation by constant cortisone administration, suggests that the response may, in this instance, reflect altered metabolism or increased "utilization" of steroids.

A Study of the Nature of the Peripheral Resistance in Patients with Hypertension. Francis S. Calrva, JaY F. Harris and Richard H. Lyons,* Syracuse, N. Y.

This study was undertaken to determine if the vascular bed of the toe participates in the increased resistance in hypertension and if the nature of this resistance differs in the various stages and types of hypertension.

Patients were of four clinical types. The first group was made up of 18 normal patients; group two, of $11 \mathrm{hy}-$ pertensive patients without eyeground changes or evidence of renal disease; group three, of 7 patients with hypertension and moderate retinopathy and renal disease; and group four, of 7 patients with marked retinopathy and/or azotemia. In none was there evidence of cardiac failure or peripheral vascular disease. Studies were carried out in a constant temperature room with the patient in the supine position. Peripheral resistances were calculated by dividing the mean blood pressure by the blood flow. Blood flows to the toe were determined plethysmographically before and after posterior tibial block.

It was found that in the resting state the peripheral resistances of each of the hypertensive groups was higher than that of the normotensives. After the abolition of local neurogenic tone, resistances of the hypertensive patients fell into three groups which correlated well with the clinical disease. The mean peripheral resistance in the normotensives after block was 7.5 units. In the hypertensive patients, group two (see above) had an average peripheral resistance of 7.2 units, group three of 12.2 units, and group four of 20.4 units.

It is suggested that the increased peripheral resistance in hypertension includes the vascular bed of the toe. This increased resistance in individuals without renal disease apparently is mainly on a neurogenic basis since it can be reduced to normal levels following a local sympathetic block. In patients with renal disease, other mechanisms must be operative.

Evidence Concerning the Relationship of the Amygdaloid Nuclear Region to the Regulation of the Circulation and Emotion in Man. William P. Chapman,* Heinz R. Schroeder, Georg Geyer, Mary A. B. Brazier, Charles Fager, James L. Poppen, Harry C. Solomon and Paul I. Yakovlev, Boston, Mass.

The effects of electrical stimulation of the amygdaloid nuclear region in the temporal lobe on the continuous recording of blood pressure and heart rate and on feeling states and motor behavior have been determined in four awake epileptic patients in whom this area was afterwards coagulated for therapeutic reasons. The patients were selected by a group of psychiatrists for psychosurgery for the treatment of psychomotor seizures or unmanageable assaultive behavior. A four-needle electrode, implanted in the amygdaloid area under stereotaxic control, made possible observations over several days as well as the localized coagulation procedure.

In one patient, no consistent responses were obtained. In three patients, electrical stimulation with $20-60$ cycles per second, 1 millisecond pulse duration and 8-14 volts produced a $20-100 \%$ increase in heart rate. In two patients, stimulation with the same electrical characteristics, except for $12-17$ volts, caused a $50-83 \mathrm{~mm}$. Hg rise in systolic and $33-60 \mathrm{~mm}$. $\mathrm{Hg}$ rise in diastolic blood pressure. The elevations in heart rate and blood pressure could be produced independently of changes in emotional expressions, but were more marked when obtained in association with feelings of anxiety and fear and somatic responses regarded as appropriate for such subjective changes. Bilateral pupillary dilation was a frequent accompaniment of the circulatory responses. The char- 
acter of the circulatory effects suggested that some responses were mediated by direct nervous pathways and others, possibly by the liberation of a humoral substance.

So far as we are aware, these findings establish for the first time that electrical stimulation of the amygdaloid nuclear region can cause marked elevations of systolic and diastolic blood pressure with or without accompanying feelings of anxiety and fear and somatic motor responses. Comparable heart rate and pupillary effects have been previously reported in animals and certain emotional changes in man, by others.

\section{A Comparison of the Cholesterol "Balance" and Nitrogen Absorption from the Human Small Intestine by an Inert Indicator Method. SAmuel H. Cheng and MaL- Colm M. Stanley,* Boston, Mass.}

A standard diet (C-250, F-103, P-116, Cholesterol-1 gram) mixed uniformly with a known amount of chromic sesquioxide $\left(\mathrm{Cr}_{2} \mathrm{O}_{8}\right)$, a chemically inert and completely non-absorbable indicator, was fed to all subjects. In six patients intestinal contents were withdrawn via a tube previously introduced under fluoroscopic guidance. Terminal ileostomy discharges were collected from a seventh. The concentrations of $\mathrm{Cr}_{2} \mathrm{O}_{3}$, nitrogen, total and free cholesterol were determined in the dried food and samples of intestinal contents. The cholesterol "balance" and nitrogen absorption were calculated from the changes in the cholesterol $/ \mathrm{Cr}_{2} \mathrm{O}_{3}$ and $\mathrm{N} / \mathrm{Cr}_{2} \mathrm{O}_{3}$ ratios.

There were negative cholesterol "balances" with simultaneous absorption of nitrogen in three subjects from whom specimens of terminal ileum contents were obtained. In the subject with an ileostomy there was, during a twoday period, with $70 \%$ nitrogen absorption, a daily net loss of $163 \mathrm{mg}$. cholesterol in excess of that ingested. Net losses of $122 \mathrm{mg}$. and $258 \mathrm{mg}$. cholesterol per day occurred in two others whose nitrogen absorption averaged 40-50\%.

In specimens obtained from upper small intestine (4 subjects) the cholesterol/ $\mathrm{Cr}_{2} \mathrm{O}_{3}$ ratios approached twice those of food and reflected the large endogenous cholesterol secretion. Cholesterol absorption in this area was thus obscured. Nitrogen absorption was $25 \%$ or less.

The cholesterol in specimens of contents of the intestine included both the unabsorbed cholesterol from the food and from that secreted in the bile and by the intestine. The cholesterol "balance" represented the sum of the endogenous and exogenous cholesterol minus that which had been absorbed. Since the amount of endogenous cholesterol secreted could not be accurately calculated from our data, the absolute amount of cholesterol absorbed from the intestine could not be determined.

Comparative Effects of Fructose and Glucose on the Fate of Infused Amino Acids and Peptides. Halvor N. Christensen, Patricia Bryan Wilber, Barbara A. Coyne and Herbert Fisher, Boston, Mass. (Introduced by Joseph M. Hayman).

The fate of infused amino acids, whether anabolic or catabolic, is known to be largely influenced by the simul- taneous availability of appropriate calorie sources. Elman et al. recently reported that more positive nitrogen balances were obtained in surgical patients when fructose rather than glucose was included in an intravenously injected protein hydrolysate. This they attributed to the well-known rapidity of the removal of fructose from the circulation. Elman also reported lower plasma amino acid levels during the course of the fructose-containing infusions.

We have investigated the influence of the type of sugar present on the distribution and fate of amino acids and peptides infused intravenously into convalescent surgical patients and normal volunteers. In the case of a fibrin hydrolysate ("Aminosol," Abbott Laboratories, Inc.) the urinary spillage of peptides was diminished from about $30 \%$ to about $13 \%$ when $10 \%$ fructose replaced 5 or $10 \%$ glucose. The wastage of free amino acids was also substantially decreased. These effects were shown to be independent of any diuretic action of the glucose solution.

In contrast to Elman's report of lower plasma amino acid levels, in our hands fructose led to slightly higher levels in 8 comparisons in 5 subjects. His use of a modified Folin colorimetric method (strongly criticized by Chinard and Van Slyke) and our use of the manometric ninhydrin method might conceivably explain this difference.

Stronger uptake of amino acids by the cells would not necessarily favor their anabolic utilization, considering that a large part of that uptake is hepatic. An inhibition of deamination and urea formation in the presence of abundant carbohydrate may instead be the factor operating to swing the balance to the anabolic side. In that case higher, rather than lower, amino acid levels would be produced in the presence of a more adequate source of energy.

The Production of Experimental Meningitis in the Rhesus Monkey. Kenneth L. Clark, Louis P. Jervey and Park BIehl, Cincinnati, O. (Introduced by Morton Hamburger).

In order to test the effect of the enzymes streptokinase and streptodornase in the liquefaction of exudate in the subarachnoid space, attempts were made to produce an experimental meningitis in rhesus monkeys. The endpoint was to be an infection in which abundant exudate would be laid down but wherein the animals would live long enough so that the effect of enzymes could be studied.

Inoculations of various bacteria suspended in gelatinLocke's solution were made into the cisternal subarachnoid space of anesthetized animals. Approximately daily cisternal taps were performed. Some of the animals were sacrificed at varying periods after infection; others died. Strains of E. coli, $H$. influenzae and Pseudomonas aeruginosa, from the spinal fluid of human cases of meningitis, Pneumococcus I, from the blood stream of a patient, Streptococcus viridans, from the blood stream of a case of subacute bacterial endocarditis, and a strain of Pneumococcus III were employed.

Pneumococcus I and E. coli produced meningitis, but killed the animals in 1 to 4 days. $H$. influenzae produced a very mild infection from which the monkeys recovered 
spontaneously. Streptococcus viridans produced pleocytosis and a positive Pandy reaction, but spinal fluid cultures remained negative and there was no exudate.

The results with Pseudomonas were irregular until a technique was devised whereby the organisms were suspended in ground up jelled agar. By this means meningitis was produced in which a thick tenacious exudate was laid down within 48 hours around the base of the brain and the cerebellum, sometimes extending several centimeters down the spinal cord. Microscopically, the exudate consisted of great numbers of polymorphonuclear leukocytes, much fibrin, and large amounts of extracellular desoxyribonucleoprotein. Spinal fluid cultures and cultures of the exudate were always positive for the infecting strain of Pseudomonas. More than $90 \%$ of the animals survived 5 days or longer.

\section{Histamincytes of the Blood-Eosinophils and Basophils.} Charles F. Code* and Ross G. Mitchell, Rochester, Minn.

This investigation was undertaken to determine whether there is a relationship between the amount of histamine and the numbers of eosinophils or basophils in the blood. The numbers of the cells and the concentration of histamine were determined quantitatively. Observations were made in dogs and human beings before, during and after the administration of cortisone.

No basophils were found in the blood of the dogs, and in these animals high concentrations of histamine occurred only in association with large numbers of eosinophils. Administration of cortisone always reduced the numbers of eosinophils in the blood, and at the same time lowered the concentration of histamine.

In human beings cortisone always caused a decline in the number of basophils as well as eosinophils. The values for blood histamine also always decreased. The numbers of basophils and eosinophils were definitely related, and they both were related to the level of histamine in the blood, indicating that they may both act as histamine carriers in the blood.

"Splanchnic Pooling" During the Hypotensive Action of Hexamethonium Bromide in the Dog. B. CoMrnsky, J. R. K. Preedy, H. O. Wheeler, R. M. Hays and S. E. BradLEy,* New York, N. Y.

The recent development of a method of estimating the circulating splanchnic blood volume (SBV) by determination of splanchnic vascular retention and dilution of $I^{\text {13 }}$ labelled human serum albumin (Trans. Assoc. Am. Phys., $66,294,1953$ ) has made possible the evaluation of the role of the splanchnic vasculature as a "blood reservoir" during systemic hemodynamic adjustments. Hexamethonium bromide ( 7.5 to $10.0 \mathrm{mg}$. ion i.v.) was used as a means of producing such an adjustment in 8 anesthetized dogs (nembutal, $30 \mathrm{mg} / \mathrm{kg}$ ) during the measurement of SBV, hepatic blood flow (BSP method), total blood volume (I ${ }^{\text {Ia }}$ HSA dilution), cardiac output (direct Fick) and arterial pressure (Statham transducer). Following administration of hexamethonium cardiac output decreased
( 15 to $76 \%$, mean-31\%) in association with a corresponding fall in blood pressure (19 to $47 \%$, mean-36\%). The total peripheral vascular resistance did not change significantly. The hepatic blood flow decreased ( $P$ less than .05 , mean-21\%) but not to the same extent as the blood pressure since splanchnic resistance was also lowered ( $P$ less than .05, mean-17\%). Despite the reduction in inflow and possibly also diminished resistance to outflow, SBV increased in 6 of 8 animals (29 to $64 \%$, mean-39\%, $P$ less than .02) suggesting an alteration in splanchnic vascular tone. Since total blood volume did not seem to change, the resultant "trapping" of blood in the splanchnic bed may have contributed to diminished venous return, leading to a lowered cardiac output and a fall in blood pressure.

C-14 Carboxyl Labeled Inulin as a Tracer for Inulin. E. Cotlove, M. C. Reder, W. B. Looney, M. Walser and L. W. MAtrhews, Bethesda, Md. (Introduced by Robert W. Berliner).

Preliminary studies have been made of the physiological behavior of radioactive inulin, prepared by Drs. H. S. Isbell and J. D. Moyer of the National Bureau of Standards by cyanohydrin synthesis, with the addition of $\mathrm{C}^{14} \mathrm{OOH}$ groups in a ratio of about 1 to 200 fructose residues. Electrophoresis of $3 \% \mathrm{C}-14$ inulin in buffer and in plasma at $\mathrm{pH} 8$ showed no migration of the inulin and no evidence of protein binding. Following intravenous injection in several species, the radioactivity was recovered in the urine completely within the error of counting, averaging $100.8 \%$ in 4 rabbits, $97.0 \%$ in 6 rats and was $101.8 \%$ in a dog and $99.0 \%$ in a man with metastatic carcinoma. Chromatographic analysis of the urine showed the radioactivity to be localized to the inulin spot. Radioactive inulin was given by continuous infusion in 4 rats; the renal clearance of the radioactivity and its volume of distribution in muscle were similar to those found previously with non-radioactive inulin. In one dog, 5 consecutive clearance periods obtained during continuous infusion of creatinine and $\mathrm{C}-14$ inulin showed a ratio of $\mathrm{C}(\mathrm{Cr})$ to $\mathrm{C}$ (radioactivity) averaging 0.95 , similar to results of Kennedy, Hilton and Berliner (Am. J. Physiol. 171: 164, 1952) with non-radioactive inulin. The volume of distribution of radioactivity in the dog was $17 \%$ of body weight.

These results indicate that the C-14 inulin has the physiological behavior of non-radioactive inulin, with no evidence of plasma binding, decarboxylation or other type of metabolism. This compound should be useful particularly in studies requiring analysis of inulin in low concentration in the presence of a chemical chromogen blank. Further investigation is in progress.

The Effect of Growth Hormone on the Amino Acid Pool and Protein Synthesis Rate in a Pituitary Dwarf. K. R. Crispell, William Parson * and Guy Hollifield, Charlottesville, Va.

The urea space, the size of the amino acid pool, and the rate of protein synthesis have been determined in five 
healthy adult volunteers using $\mathrm{N}-15$ glycine and $\mathrm{N}-15$ urea by the method of San Pietro and Rittenberg.

The same type of study has been carried out in a 20 year old dwarfed, sexually immature male who had undergone pituitary surgery at the age of eight. The patient was studied under the usual standard metabolic regimen. Before the administration of growth hormone (Raben) the patient was found to have an amino acid pool of $3 \mathrm{mg} . / \mathrm{Kg}$. body weight as compared to $14 \mathrm{mg}$. $/ \mathrm{Kg}$. (range 11 to 18 ) in the healthy volunteers. The rate of protein synthesis was $290 \mathrm{mg}$. $/ \mathrm{Kg}$. per 24 hours as compared to $810 \mathrm{mg}$. $/ \mathrm{Kg}$. per 24 hours (range 610 to 1170 ) in the healthy volunteers.

The patient was then given purified growth hormone (Raben), $20 \mathrm{mg}$. every six hours intramuscularly and the studies repeated on the sixth day of administration. Classical balance studies on this date showed minimal nitrogen retention. The urinary nitrogen averaged $5.0 \mathrm{Gms}$. daily for the six days previous to growth hormone, and 4.4 Gms. daily for the six days while receiving growth hormone. There was, however, a profound change demonstrated by the isotope technique. The amino acid pool increased from $3 \mathrm{mg} . / \mathrm{Kg}$. to $11 \mathrm{mg}$. $/ \mathrm{Kg}$. and the rate of protein synthesis from $290 \mathrm{mg} . / \mathrm{Kg}$. per 24 hours to $1,180 \mathrm{mg} . / \mathrm{Kg}$. per 24 hours.

This technique appears to be more sensitive than classical nitrogen balance studies in assaying changes in protein metabolism. It may also offer some insight into the mechanisms occurring with hormonal manipulation.

\section{The Comparative Effect of Platelets on Prothrombin Uti-} lization from Dogs in the Degenerative and Regenerative Phase of Irradiation Bone Marrow Aplasia. E. P. CRONKITE and G. J. JACOBS, Bethesda, Md. (Introduced by L. M. Tocantins).

A quantitative relationship between platelet concentration and prothrombin utilization in plasma of normal dogs has been shown by Buckwalter $e t a l$. and in whole blood of irradiated dogs by Jackson et al. In the latter studies, it appeared that the irradiated platelets were less effective than normal platelets in accelerating prothrombin conversion. In contrast the platelets in whole blood of dogs in the regenerative stage appeared more effective than platelets in the degenerative stage (Cronkite et al.). These phenomena might be explained on the basis of changes in the platelets or changes in concentration of plasma anticoagulants (antithromboplastins).

To investigate these phenomena, the influence of the following on prothrombin utilization was studied:

1. The comparative effect of platelets in whole blood in the degenerative stage with platelets in the regenerative stage.

2. The comparative effect of platelets in plasma from normal dogs and in plasma from dogs in the degenerative phase by varying platelet count with differential centrifugation and silicone technique without anticoagulants.

The following relationships were demonstrated:

1. At all platelet levels, platelets in the degenerative phase of radiation injury were less effective in accelerating prothrombin conversion than platelets in the regenerative phase when the conversion took place in whole blood.

2. In plasma at similar platelet levels, platelets of irradiated dogs in the degenerative phase were less effective than normal platelets.

3. In both cases above, platelets in the degenerative phase were less effective by a factor of two or three.

4. Technically, it was not possible to cross-transfer irradiated or normal platelets to the opposite plasma and obtain meaningful results. It was therefore not possible to ascribe these alterations in platelet behaviour primarily to a plasma constituent or to qualitative changes in the platelet but previous work by Tocantins strongly suggests that lipid antithromboplastins may partly contribute to the lesser effectiveness of platelets in the degenerative stage.

The Renal Oxygen Consumption in Diabetic Nephropathy as Related to Tubular Functional Mass. ARCHER P. Crosley, Jr., George G. Rowe, John H. Huston, Grorge M. Maxwell and Charles W. Crumpton, Madison, Wis. (Introduced by Edgar S. Gordon).

The ratio of renal oxygen consumption (total renal blood flow $X$ arterial-renal venous oxygen difference) to renal tubular mass $\left(\mathrm{Tm}_{\mathbf{P A B}}\right)$ in 4 patients with diabetic nephropathy has averaged 0.659 . This value represents a threefold increase from that observed in normal patients $(0.232)$. The specificity of this elevation to diabetic nephropathy is reflected in further studies indicating that it is significantly different from results obtained in patients with essential hypertension $(0.216)$, labile diabetes without nephropathy $(0.217)$, and the presence of poor renal function $(0.070)$.

These results demonstrate a unique renal metabolic pattern in diabetic nephropathy which may be related to other endocrine abnormalities associated with this condition.

\section{Homeostatic Limits in Patients with Chronic Nephritis} and Therapeutic Implications. JOHN D. CRAWFORD, Gerald A. Kerrigan, Winston E. Cochran, Mary Terry and Nathan B. Talbot,* Boston, Mass.

Studies have been performed in a small series of patients with advanced chronic nephritis to define their residual ranges of renal capacity for homeostatic adjustment. The study technique involved altering rate of input of selected individual constituents of intake to provide challenge situations. In such circumstances the adaptive behavior of the patients has been described and limits of renal capacity for homeostatic adjustment have been taken as those points at which further compensatory changes in urinary excretion fail to occur while pathologic deviation in body content or concentration of the constituent under study develops. Limits have been described for urine water concentration and volume, $\mathrm{pH}$ and ammonium ion content and for the excretion rates of sodium, potassium, phosphate and urea.

These studies have indicated certain important differences in the impairments shown by individual patients. 
They have shown major reductions in capacity to vary urine water concentration. Urine volumes greater than thirty per cent of the glomerular filtrate may be formed. Renal ability to conserve sodium and potassium has been found unimpaired except in acidosis when fixed cation losses result from reduced excretion capacity for hydrogen and ammonium ions. Maximum excretory capacities for potassium and phosphate are diminished in proportion to glomerular filtration, but their excretion, like inulin and urea clearances, may be augmented by large sodium intakes.

The definition of these residual renal capacities has facilitated design of individualized diets which permit restoration and maintenance of normal body composition. This has been followed by changes including improvement in well-being, resumption of growth, healing of rickets and reduction of hypertension. While the degree of alteration in ultimate prognosis referable to such therapy is still uncertain, the results obtained to date suggest that this method of approach may prove both of practical and basic interest.

The Visceral Circulation in Patients with Coarctation of the Aorta. James W. Culbertson, ${ }^{*}$ W alter M. KIrKendall, John W. Ecrstein and George N. Bedeli, Iowa City, Ia.

Available reports on renal and total systemic (= pulmonary) blood flow in patients with coarctation of the aorta are conflicting and often fragmentary. Hepatic (= splanchnic minus renal and adrenal) circulation remains unrecorded.

We have studied renal circulation in 14 patients, 10 of them having cardiac output determinations also, and 8 of these having additional hepatic blood flow estimations. Standard hemodynamic (Fick principle) and plasma clearance chemical methods were employed, including direct registration of intra-arterial pressure levels from both brachial and femoral arteries.

Seven patients had elevated cardiac indices (4.12$\left.5.56 \mathrm{~L} / \mathrm{min} . / \mathrm{M}^{2}\right), 1$ a normal value $(3.04)$ and 2 in congestive myocardial decompensation subnormal values (2.24 and 1.56). Renal plasma flow was elevated ( 971 $\mathrm{ml} / \mathrm{min}$.) in 1 , normal (553-697) in 8 , and subnormal in 5 (of whom 3 were in heart failure). Only these 5 had increased renal vascular resistance. Hepatic hyperemia was found in $3(2037-2559 \mathrm{ml} / \mathrm{min}$.), high normal blood flow $(1545-1866)$ in 3 , and low normal (1145 1186) in 2 who were in subclinical heart failure. Splanchnic vascular resistance was normal in these 2 but reduced in 6 . Bromsulfalein plasma clearance $\left(C_{\text {bop }}\right)$ was normal $(441-757 \mathrm{ml} / \mathrm{min}$.) in 7 , low (142) in 1 and elevated (964) in 1 . Splanchnic oxygen consumption $(85-113 \mathrm{ml} / \mathrm{min}$.) averaged above normal, as did total oxygen consumption. Systolic arterial hypertension was found in the upper compartment and normal mean arterial pressure in the lower.

The data fail to show significantly increased arteriolar resistance locally or generally, diastolic hypertension, or renal ischemia in patients not in myocardial decompen- sation. They do support normal renal function, hepatic hyperemia with decreased splanchnic arteriolar resistance, and increased total blood flow and general and splanchnic metabolism. This is in sharp contrast to the hemodynamics of essential arterial hypertension.

Comparative Absorption Rates in the Upper and Lower Human Small Intestine. Alvin J. Cummins and RaIne Jussil A, Philadelphia, $\mathrm{Pa}$. (Introduced by Thomas E. Machella).

Data from animal experiments comparing absorption rates in various levels of the small intestine are meager and conflicting. Similar data are not available for the human. Because of the possible significance of such knowledge in various clinical conditions, comparison studies of absorption rates in man were undertaken.

The intubation technic of Nicholson and Chornock was used, permitting measurement of the absorption rate of a $45 \mathrm{~cm}$. segment of the small bowel. In the present study, rate of absorption in the upper intestine was compared with that in the lower ileum in the same patient, the tests being run consecutively. Glucose solutions in concentrations of $5 \%$ and $10 \%$ were used as test substance in seven and six normal subjects, respectively. Urea absorption from solutions of similar tonicity was also determined in three subjects.

The resulting data indicate a mean absorption rate in the ileum $29 \%$ below that of the upper intestine for $5 \%$ glucose and $46 \%$ less for $10 \%$ glucose. Reduced absorption rates of similar magnitudes in the lower bowel were also demonstrated for urea. The results further suggest that the absorption rate for water may be reduced in the ileum, as indicated by the larger volumes of fluid aspirated there.

Failure to demonstrate significant difference in the concentration of unabsorbed glucose aspirated from either site implies that reduced ileal absorption rate is not due to greater dilution. Likewise, comparable absorption rates of glucose and the readily diffusible substance urea make it unlikely that the observed difference is due to variation in mucosal phosphorylating capacity. Balloonkymographic studies during the performance of the tests confirm the previously observed gradient of lessened motility from upper to lower intestine and this motor difference may possibly be implicated in the variation in absorption rate.

Observations on Spontaneous and Experimental CheyneStokes Breathing in Man. JAMrS H. CuRRENS and Rhetr TAlBert, Boston, Mass. (Introduced by Mandel E. Cohen).

Cheyne-Stokes breathing is characterized by recurring periods of apnea and subsequent respirations which wax and wane. A systematic study of this phenomenon has been in progress which has endeavored to (1) record the respirations, (2) study arterial blood chemistry in different phases, (3) evaluate cardiac, neurological and mental status and (4) test the effect of drugs and stimuli.

Eleven patients have been observed to have spontaneous 
or induced Cheyne-Stokes breathing. The breathing was recorded in five patients while inhaling 100 per cent oxygen, and in one patient Cheyne-Stokes breathing was observed with a persistent blood oxygen saturation of 100 per cent. Stimuli such as pain, noise, attention, increased awareness and carbon dioxide have all been found to alter or dispel the Cheyne-Stokes breathing. Conditions which have enhanced this breathing have been relaxation and sleep. In three patients this breathing occurred after the lowering of blood pressure from protoveratrine or hexamethonium. Aggravation of abnormal neurological signs was observed concomitantly with Cheyne-Stokes breathing. The constant dispelling of this phenomenon with carbon dioxide inhalation has been striking; this is contrasted with the fact that oxygen usually had no effect.

Nine cases have had abnormal neurological signs or a history of apoplexy. Four autopsied cases have each demonstrated cerebral injury, usually bilaterally. One of the autopsied cases had no abnormal neurological signs.

It is concluded that emphasis should be placed upon the neurological aspect of this type of breathing rather than on blood chemical changes or degree of congestive heart failure. The first description of this disorder by Cheyne in 1818 was a cardiac patient who experienced apoplexy which rendered him "speechless and hemiplegiac" and whose breathing "would entirely cease for a quarter of a minute then by degrees become heaving and quick."

Inoculation Syphilis in Man. JoHN C. CutLer, LOPO DE Mello, Bernard I. Kaplan, Harold J. Magnuson,* Sidney Olansky and Evan W. Thomas, Chapel Hill, N. C.

Sixty human prisoner volunteers with well documented medical histories were inoculated intracutaneously with the Nichols strain of $T$. pallidum. The experiment was designed to assess immunity in relation to serologic tests with lipoidal antigens, treponemal immobilization test, and duration of original infection prior to treatment. Eight of the subjects were non-syphilitic, the remainder previously had had syphilis and all except 4 had been treated for the disease. Prior to challenge inoculation one half the group received $5 \times 10^{3}$ heat killed $T$. pallidum intracutaneously and subcutaneously. An anamnestic T. P. I. response was demonstrated in some patients, but reagin titers were unchanged. Graded inoculums ranging from 10 to $10^{4}$ virulent organisms were inoculated intracutaneously at 4 sites in each of the controls. The $50 \%$ infectious inoculum was found to be between 10 and $10^{2}$ organisms. All other subjects were challenged at a single site with $10^{5}$ organisms.

The early course of the disease in the normal controls was analogous to that observed in naturally acquired infection as measured by clinical and serologic changes. Of 11 patients previously treated for early syphilis, 9 developed dark field positive lesions, 2 dark field negative lesions. All showed increasing titers of reagin and T. P. I. antibody. Of 34 patients previously treated for late syphilis, 2 developed dark field positive lesions, 12 dark field negative lesions with increasing serologic titers, 3 dark field negative lesions without serologic change, and 16 showed no clinical or serologic evidence of infection. Two of the lesions were clinically gummata. None of the 4 untreated patients showed clinical or serologic response to inoculation. One patient previously treated for asymptomatic neurosyphilis had as sole response a redevelopment of active asymptomatic neurosyphilis. This patient represents the only reinfection in which no cutaneous lesion developed at site of inoculation. All patients were treated with penicillin no later than 4 months after inoculation.

Acquired immunity in human syphilis is shown to exist and appears to be related to the duration of the original immunizing infection prior to its treatment.

The Character of the Records Obtained from the Aperiodic Ballistocardiograph and Their Relationship to Those from the High-Frequency Bed (Starr) Ballistocardiograph. D. C. Deuchar, S. A. TALbot, F. W. Davis, JR. and W. R. Scarborough, Baltimore, Md. (Introduced by Benjamin M. Baker, Jr.).

Analysis of the high-frequency bed ballistocardiograph has demonstrated defects of this method of recording motions of the body resulting from cardiovascular forces. Important amongst these is the excitation of the spring and damper system formed by the dorsal tissues of the subject, resulting in a distorted frequency response in the ballistocardiographic range.

A light platform floating on mercury has been constructed and ballistocardiograms have been recorded using an accelerometer. This system has no restoring force, so is aperiodic, and has minimal damping. These properties minimize excitation of the dorsal tissues and approximate the ideal of a freely floating body. Acceleration records so obtained measure forces referred to the body without distortion through most of the ballistocardiographic spectrum now known. Mathematical analysis suggests that displacement records from the high-frequency bed represent grossly the slower forces acting on the body, so the two types of records can justifiably be compared to study the distortion present in the latter.

Records of 60 subjects, normals and abnormals, have been obtained from both systems. Subjects with normal high-frequency bed records yield a consistent pattern on the aperiodic bed which contains slurs, splinters and faster detail, with the major waves slightly preceding the high-frequency bed record in time; late systole typically shows a series of small forces, without a major footward contribution, in place of the $K$ wave which we therefore regard as artefact, in normal records, due to after-oscillation. Diastole also shows consistent welldefined forces. Abnormal records demonstrate well a similar, consistent relationship to the high-frequency bed record in all cases. This supports the view that the highfrequency bed displacement ballistocardiograph reflects grossly the force on the body but significantly distorts its detail both in timing and in wave form. 
Erythrocyte Metabolism. Studies on the Metabolic Behavior of Reduced Glutathione in Human and Avian Erythrocytes. Elmanan Dimant, Edith Landsberg and Irving M. London,* New York, N. Y.

In studies on the ageing process in erythrocytes, the patterns of metabolic behavior of various constituents of the erythrocytes are under investigation. This report is concerned with reduced glutathione, which has been implicated in the maintenance of integrity of the structure of the erythrocyte.

Glycine labeled with $\mathrm{N}^{16}$ was administered to a normal man, and glutathione and hemin were isolated from the erythrocytes at intervals thereafter. The isotope concentrations in the hemin reveal a normal pattern of survival of erythrocytes. The isotope concentrations in the reduced glutathione indicate that the nitrogen, derived from glycine, of the reduced glutathione in the human erythrocyte is in the dynamic state with a half-time value of approximately four days.

Incubation, in vitro, of intact or lysed human or duck erythrocytes with 2-C $\mathrm{C}^{\mathbf{1 6}}$-glycine results in rapid incorporation of the labeled carbon in the reduced glutathione of the erythrocytes. These findings indicate the existence of a system in the mature erythrocyte for continued replacement of the glycine moiety of reduced glutathione and provide a basis for the study of the origins, and the mechanism of reduction, of glutathione of erythrocytes.

The Effect of Tetraethylammonium Chloride on the Pressor Response to Epinephrine and Norepinephrine. R. J. DiSalvo, James D. Evans and Albert A. Brust, Atlanta, Ga.

The phenomenon of potentiation of the pressor effects of epinephrine, norepinephrine and other pressor substances during autonomic blockade with tetraethylammonium chloride (TEAC) has been extensively studied in animals. In view of the lack of similar controlled studies of this phenomenon in man, this study was undertaken in order to determine whether similar responses occurred in human subjects, since such responses may be a factor in the pressor response to TEAC in pheochromocytoma, and to aid in clarifying the interplay of neurogenic and humoral factors in the regulation of vascular tone.

Comparative studies of the blood pressure and pulse effects of rapid intravenous injection of TEAC before and during constant intravenous administration of 1-epinephrine and 1-norepinephrine have been made in six normotensive subjects. Blood pressure was measured by the auscultatory method; in 3 subjects direct arterial pressures were also recorded from a brachial artery by a Statham pressure transducer and strain gauge amplifier.

During a control period, a rapid injection of $400 \mathrm{mgm}$. of TEAC was given, intravenously. When the effects of the drug disappeared, intravenous infusion of $.085 \mathrm{mcg} . /$ $\mathrm{Kg} . / \mathrm{min}$. of epinephrine was begun. During the infusion, when blood pressure and pulse stabilized, $400 \mathrm{mgm}$. of
TEAC was again given intravenously. In the same subjects on a subsequent day, the procedures were repeated except that $.085 \mathrm{mcg} . / \mathrm{Kg} . / \mathrm{min}$. of norepinephrine was given.

In all subjects a depressor response to TEAC occurred in both control periods (mean $-22 /-12$ ). The response to TEAC remained depressor, although of less magnitude, during epinephrine infusion. During norepinephrine infusion, a pressor response to TEAC occurred in all subjects (mean $+20 /+12$ ), and the bradycardia due to norepinephrine was abolished.

The results indicate that in the range of the physiologic doses used, TEAC augments the pressor effects of norepinephrine, but not of epinephrine, and suggest that the mechanism of the pressor response to TEAC in pheochromocytoma may be due to the presence of circulating norepinephrine.

The Exophthalmos-producing Substance in the Serum of Patients Suffering from Progressive Exophthalmos. Brown M. Dobyns* and LoIs A. Wilson, Cleveland, $\mathrm{O}$.

It has been known for a long time that crude anterior pituitary extracts will produce both thyroid hyperplasia and exophthalmos when injected into the animals. We have recently shown that the exophthalmos producing substance (EPS) is different from the thyroid stimulating hormone. By chemical methods these two substances have been separated and their actions independently demonstrated.

Fundulus, an Atlantic minnow, has proven to be an excellent test object for the exophthalmos producing substance. Amounts of dried extracts as small as 25 micrograms will cause a positive exophthalmic response in these fish. The response usually can be recognized grossly in 3 to 6 hours, but it may be accurately determined by measuring the intercorneal distance in each fish before and following administration of the test material.

After testing whole serum from many patients with severe or progressive exophthalmos in the past with only borderline or negative responses, we have recently observed that the serum of 3 such patients caused a positive response when administered to the fish.

In contrast to the prompt response described above, the response from whole serum usually required several days. Because the volume of fluid that can be injected into the coelomic cavity of these fish is small, 3 injections of $0.25 \mathrm{ml}$. of serum are given over a period of 24 hours. Some of our previous failures to demonstrate an exophthalmos producing substance in whole serum have probably been due to 1) an insufficient period of observation following injection, 2) insufficient amounts of whole serum given (single doses), and 3) marked variation in responsiveness of lots of fish throughout the year. Control observations, using normal saline, normal saline containing a standard active EPS pituitary preparation and pooled normal serum, have been made. 
Resistance to Airflow through the Tracheobronchial Tree as Measured by Means of a Body Plethysmograph. A. B. DuBois, S. Y. Botel ho and J. H. Comroe, JR., Philadelphia, $\mathrm{Pa}$.

Since the ratio of pressure/flow measures resistance, in order to obtain resistance to airflow through the tracheobronchial tree, it is necessary to have simultaneous measurements of airflow and alveolar pressure during the period of flow. This is accomplished by having a subject sit in an airtight body plethysmograph and breathe air from the plethysmograph through a flowmeter. Pressure changes in the plethysmograph are measured continuously during the respiratory cycle and represent alveolar volume changes caused by compression or decompression of the alveolar gas during expiration and inspiration. For example, for gas to flow out of the alveoli during expiration, the alveolar pressure must rise above atmospheric; if at any one instant the absolute alveolar gas volume is $3000 \mathrm{ml}$ and the alveolar pressure rises to $10 \mathrm{~cm} \mathrm{H} \mathrm{H}_{2} \mathrm{O}$, the change in alveolar gas volume, due to compression, is $30 \mathrm{ml}$. Since such small changes in alveolar gas volume during airflow can be detected by the change in pressure in the body plethysmograph and one other unknown, the absolute alveolar gas volume can be obtained by measuring simultaneous change in alveolar volume and pressure in the tracheobronchial system during the period when flow has been interrupted by a shutter, alveolar pressure during airflow can be calculated. Measuring simultaneous alveolar pressure and airflow in this manner, we have found the resistance of the tracheobronchial tree to airflow to be $1.63 \mathrm{~cm} \mathrm{H}_{2} \mathrm{O} / \mathrm{L} . / \mathrm{sec}$. \pm S.D. 0.36 in 15 normal subjects. In subjects with increased airway resistance, the values are considerably higher.

Metabolism of Compound $S$ in Rheumatoid Arthritis; Effect on 17-Ketosteroid Excretion. IvaN F. DUFF, William D. Robinson, William M. Mikrelsen and Aletha M. Hemerline, Ann Arbor, Mich. (Introduced by J. W. Conn).

Recent studies (J. Lab. \& Clin. Med., Jan., 1954) by Conn, Fajans, Louis, Seltzer suggest that the excretory level of 17-ketosteroids can be modified by altered metabolism of adrenocortical steroids, and disclosed that patients with liver disease excrete a much smaller proportion of administered Compound $S$ as 17-ketosteroids than do normal individuals. In normal subjects, on the second consecutive day of Compound $\mathrm{S}$ administration orally in doses of $400 \mathrm{mgm}$. daily, there is a peak increase in urinary 17-ketosteroid excretion of 25 to $45 \mathrm{mgm}$. above baseline values.

This test has been applied to 6 men and 6 women with rheumatoid arthritis. Baseline excretion of 17-ketosteroids was below normal in 10 of these 12 . In only 4 patients did oral administration of compound $\mathrm{S}$ produce a normal increment in 17-ketosteroid excretion (23 to 73 mgm.) ; in one subject the response was $17 \mathrm{mgm}$. In the remaining 7 , the rise in excretory level was $12 \mathrm{mgm}$. or less, with no increase whatsoever in 2 subjects. Further alteration in excretory pattern characterized by delay in peak values was seen in 5 patients. Liver function, as measured by serum bilirubin, urinary urobilinogen and bromsulphophthalein retention, was normal in these patients, with the exception of excessive BSP retention in one. The abnormal serum protein pattern commonly observed in rheumatoid arthritis was present in 8 . There was no obvious correlation with duration, severity or extent of the disease process.

These preliminary results suggest that further investigation of the metabolism of adrenocortical steroids in rheumatic and other chronic diseases may be informative.

Some Differences in Ventricular Contraction and Relaxation in Patients with Mitral Stenosis and Mitral Insufficiency as Studied by the Kinetocardiogram. E. E. Eddleman, JR. and Robert H. YoE, JR., Birmingham, Ala. (Introduced by T. R. Harrison).

Low frequency precordial chest movements (kinetocardiograms) were recorded in patients with mitral stenosis and mitral insufficiency by means of a bellows system previously described. The events were correlated with the electrocardiogram, carotid pulse, and ballistocardiogram.

Patients with mitral stenosis revealed a marked anterior or outward movement of the precordium occurring before ejection which was sustained above the baseline during systole. This was followed by an inward movement during isometric relaxation. In contrast to this, patients with mitral insufficiency had an inward movement of the chest during early ejection followed by a large outward movement just before the carotid incisura.

The findings were interpreted as indicating an exaggerated anterior swing of the heart during systole in mitral stenosis probably as a result of the contraction of the right ventricle. The swing predominates during ejection. However, as the right ventricle releases, the left ventricle pulls the heart posteriorly and leftward in the chest. Mitral insufficiency appears to lack the early marked anterior swing but has a late systolic swing of the heart. Thus, there appear to be fundamental differences in ventricular contraction and relaxation in patients with mitral stenosis and mitral insufficiency.

The Effect of Myocardial Anoxia on Myocardial Metabolism in Vivo. Sterling Edwards, A. Stegel and Richard J. Bing,* Birmingham, Ala.

The effect of myocardial anoxia resulting from hemorrhagic shock was studied in 16 anesthetized dogs. Shock was produced with a modified technique of Wiggers (blood pressure reduction to $50 \mathrm{~mm}$. $\mathrm{Hg}$ for 90 minutes, then $30 \mathrm{~mm}$. $\mathrm{Hg}$ for 60 minutes, followed by reinfusion of blood). Coronary blood flow and myocardial extraction and usage of oxygen, glucose, pyruvate and lactate were determined during the control period and during the oligemic and normovolemic phase. During the oligemic phase, coronary flow, coronary vascular resistance, myocardial oxygen consumption and mechanical efficiency were reduced. Reinfusion of blood did not result in a 
return of myocardial oxygen consumption to normal control values. The most significant observation was a reversal of the usual positive pyruvate balance during the oligemic phase with the concentration of pyruvate in coronary vein blood significantly exceeding that in coronary artery blood. This was accompanied in some instances by similar reversal in the extraction of a reducing substance determined as glucose by the micromethod of Hagedorn. Myocardial oxygen extraction of lactate remained positive throughout. These results indicate that reduction of coronary blood flow results in severe myocardial anoxia which may be responsible for (1) inhibition of enzyme systems engaged in the breakdown of pyruvate (cocarboxylase); (2) increased glycolysis, which may represent the prime source of cardiac energy under those conditions. As a result, pyruvate accumulates. Continued extraction of lactic acid might result from conversion of lactate to pyruvate or its conversion to carbohydrate intermediates in the direction of glycogen. These metabolic changes point to the possibility of an anaerobic metabolism of heart muscle in vivo.

The Effect of Congestive Heart Failure on Total Blood Volume, Red Cell Mass, and Plasma Volume as Measured by Radioactive Chromium-Labeled Red Cells. Seymour EIsenberg, Dallas, Tex. (Introduced by Elias Strauss).

The effect of congestive heart failure on total blood volume and circulating red cell mass remains a matter of considerable controversy. Measurements with T-1824 dye have yielded consistently increased values but it is likely that they included an expanded lymphatic space. Measurements utilizing radiophosphorus have yielded inconsistent changes, but the instability of the $\mathrm{P}-32$ red cell complex precludes satisfactory equilibration periods.

Determination of blood volume and circulating red cell mass with radiochromium labeled red cells suffers from neither of these defects. This method has therefore been utilized in the study of 27 subjects with congestive heart failure. Comparisons were made between pre-treatment and post-treatment values and between these and the values of 20 normal subjects. The patients were grouped according to the "degree' of congestive failure, dependent upon duration, severity, and tractability.

Congestive heart failure resulted in a $22 \%$ increase in blood volume, a $25 \%$ increase in red cell mass and a $17 \%$ increase in plasma volume. There was a $38 \%$ increase in blood volume in the "severe" group as opposed to a $14 \%$ increase in the "mild" group. After treatment, the blood volume decreased in $50 \%$ of the patients. This shrinkage involved a reduction in both red cell mass and plasma volume; only rarely did selective shrinkage of plasma volume occur. The magnitude of hypervolemia was correlated with the degree of cardiomegaly; following treatment, shrinkage of blood volume tended to parallel the decrease in heart size.

It is concluded that:
1. Heart failure is usually accompanied by an expanded blood volume, which shrinks after compensation is restored.

2. The expanded blood volume, as well as the reduction following compensation, involves red cells and plasma equally.

3. Changes in blood volume parallel changes in heart size and are not apparently related to the formation or delivery of edema.

The Effect of Variations in Amino Acid Intake and Protein Deficit on the Metabolic Response of Soft Tissues and Bone to Cortisone Acetate. Leon ARD P. ElIEL * and Robert P. Heaney, Oklahoma City, Okla.

The effects of variations in protein anabolic rate on the metabolic response to oral cortisone acetate, 200 to 600 mg./day, have been studied in a patient who was fed synthetic isocaloric diets of differing amino acid content.

On a zero amino acid intake, after urinary nitrogen excretion had stabilized at about $2.0 \mathrm{gm}$./day, administration of cortisone resulted in a transient, slight increase in nitrogen excretion, averaging $0.1 \mathrm{gm}$./day. Amino acids $50 \mathrm{gm} . / \mathrm{day}$, and cortisone, were given during a subsequent period of marked positive nitrogen balance. A slight negative balance was achieved only on high doses of cortisone. With an amino acid intake of $100 \mathrm{gm}$./day, after establishing nitrogen equilibrium, cortisone resulted in a prompt and sustained negative nitrogen balance, averaging $1.5 \mathrm{gm} . /$ day.

Urinary calcium, during administration of cortisone, rose 96 and $173 \mathrm{mg}$./day, at the zero and $50 \mathrm{gm}$. amino acid levels, respectively. Preexistent hypercalcuria confused interpretation of a rise at the $100 \mathrm{gm}$. level. The serum alkaline phosphatase concentrations fell significantly at all amino acid levels when cortisone was exhibited.

Retention of sodium, chloride and water, and eosinopenia, were manifested at all amino acid levels, and independently of the nitrogen balance, during administration of cortisone.

The results provide further evidence that the principal effect of cortisone on protein metabolism is antianabolic. However, in the presence of a strong anabolic stimulus (protein deficit plus adequate amino acid intake), administration of cortisone failed to induce nitrogen losses, except in very high doses. The increases in urinary calcium, during administration of cortisone, indicated either that bone anabolism continued, to a small degree, on an amino acid-free diet, and was inhibited, or that cortisone also stimulated bone catabolism.

Plasma Mixing and Removal Curves for $F e^{50}$ and $I^{131}$ After Intravenous Injection of Doubly Labeled Iron Binding Globulin. P. J. ElMLINGER, S. P. MAsouredis, J. Soni, G. E. Fulton and S. L. Belknap, Berkeley, Calif. (Introduced by John H. Lawrence).

Studies of iron turnover involving the labeling of total plasma iron have aroused interest in the metabolic relationship between the circulating plasma iron (CPI) and its globulin carrier. The turnover rate of the iron binding 
globulin (IBG) as well as the plasma iron turnover rate was determined with $\mathrm{Fe}^{\mathrm{s}}$ globulin in which the protein was trace labeled with $\mathrm{I}^{13}$.

In patients given the doubly labeled IBG the two isotopes in the serial samples were separated chemically prior to radioassay. IBG singly labeled with $\mathrm{I}^{\mathrm{m}}$ was used in some patients for comparison of IBG turnover rates with iron turnover rates determined several months earlier.

Extrapolation to zero time of the first post-mixing component was used for defining space of dilution of $\mathrm{Fe}^{\mathrm{m}}$ and $I^{121}$ IBG. Turnover rates of iron and of the IBG were determined from their respective half-times, the concentration, and volume.

The serial changes in body distribution of radioactivity after injection of IBG labeled only with $I^{12}$ are similar to those observed with gamma emitters confined within the vascular bed and are in contrast to the changes which occur during the first three weeks following the injection of IBG labeled with $\mathrm{Fe}^{50}$. It was calculated that 0.1 to 0.2 of the total IBG was replaced per day which differs markedly from the replacement rate of the total iron.

The above data are compatible with the concept that the globulin functions as a carrier for iron. In addition, the data suggest that some abnormalities in the siderokinetic pattern in various disease states are reflections of disturbed carrier globulin metabolism.

Studies of the C-Reactive Protein in the "Post-Commissurotomy" Syndrome. SAMUEL K. ELSTER and HARRISON F. Wood, New York, N. Y. (Introduced by Alexander B. Gutman).

The "post-commissurotomy" syndrome developed in eight of thirteen patients with rheumatic mitral stenosis two weeks to seven months after commissurotomy. The characteristic chest pain, fever and cough, often with signs of pluritis and pericarditis, usually lasted 1-3 weeks. Multiple attacks occurred in 3 patients.

The C-reactive protein (CRP) in the sera of twelve patients was determined pre- and post-operatively. The CRP became positive within 24 hours post-operatively and remained elevated 1-8 weeks. Markedly elevated titers $(4-6+)$ occurred in ten patients. Control studies in seven patients following surgery for congenital heart disease revealed similar high post-operative titers of CRP but disappearance within a week in six.

Of the eight patients with the "post-commissurotomy" syndrome, four had transiently positive CRP pre-operatively; three had auricular biopsies positive for "Aschoff bodies." Of the remaining five patients, one had a positive CRP pre-operatively and two had positive auricular biopsies.

Serial determination of the CRP was performed in six instances of the "post-commissurotomy" syndrome. In all, the CRP became positive at onset and persisted throughout the period of clinical activity. As the syndrome remitted, the CRP diminished and in four instances persisted for variable periods following apparent clinical remission. In three cases, salicylates appeared to suppress the clinical manifestations. In one, this was associated with rapid subsidence of the CRP. Following withdrawal of the salicylates, the CRP reappeared promptly and without any further treatment slowly subsided.

The consistent appearance of CRP in the "post-commissurotomy" syndrome does not indicate that this disease necessarily represents a manifestation of rheumatic fever. The determination appears to be useful in the diagnosis and management of the "post-commissurotomy" syndrome.

Studies of an Unusual Patient with Plasmacytosis. Ralph L. Engle, Jr. and Lila A. Wallis, New York, N. Y. (Introduced by David P. Barr).

A 38 year old man was found to have $40 \%$ plasma cells in the bone marrow and an occasional one in the peripheral blood. Almost all of the cells contained rod- or rectangular-shaped cytoplasmic inclusion bodies which took the azur stain. The serum protein was $5.4 \mathrm{gm} . \%$ with an $A / G$ ratio of $4.1 / 1.3$. Filter paper electrophoresis revealed a marked hypogammaglobulinemia. The urine contained large quantities of a protein resembling BenceJones protein which precipitated out upon heating and redissolved upon boiling. This protein migrated as betaglobulin on filter paper electrophoresis. There was hepatosplenomegaly and a moderate anemia. $\mathrm{X}$-rays of the bones showed minimal osteoporosis. Two small rib fractures were present. A diagnosis of multiple myeloma was entertained.

There were, however, other features not readily explained by the diagnosis of multiple myeloma, but more characteristic of the adult Fanconi syndrome. Among these was a constant aminoaciduria, measured by twodimensional paper chromatography, which was not found in 11 patients with multiple myeloma. In addition, glycosuria of renal type, hypocalcemia, hypophosphatemia, and an elevated alkaline phosphatase were found.

The urinary protein differed from the form described by Bence-Jones in that at $\mathrm{pH} 5$ it precipitated out at $65^{\circ} \mathrm{C}$ rather than at $45-55^{\circ} \mathrm{C}$. Studies of the amino acid composition of this protein by means of two-dimensional paper chromatography revealed the presence of methionine in considerable amounts in contrast to typical Bence-Jones protein which in our experience and that of others contained practically no methionine.

The Serum Precipitable Iodine (SPI) in Surgical Stress and Serious Illness. WILliaM W. Engstrom and Blanch Markard, Milwaukee, Wis. (Introduced by Randall G. Sprague).

The following studies were undertaken to determine whether surgical stress elicits effects which alter thyroid function (as determined by the SPI) and to define more clearly the limits of the SPI as a diagnostic technic. In 21 of 24 operated patients no significant change in the SPI occurred either acutely (hours) or during one week after surgery. Thus the phenomena associated with surgical stress do not appear to be directly related to al- 
terations in the concentration of thyroid hormone in serum. However, the SPI progressively fell to abnormally low levels in three patients who expired 5, 6 and 19 days post-operatively.

The behavior of the SPI was then studied in 32 seriously ill patients who suffered from a variety of conditions. Fourteen of these expired; the remainder survived during the period of observation. The SPI varied from 1.2 to 5.9 micrograms \% (mean: 3.2 micrograms); these values contrast sharply with our control range of 3.5 to 7.0 micrograms \% (mean: 5.1 micrograms). The abnormally low values appeared to be related to some phenomenon of the illness itself rather than to survival or to acute stress. Similar to the findings of Recant and Riggs (J. Clin. Investigation $31: 789,1952$ ) in patients with nephrosis, we found a close correlation between the SPI and the level of the serum albumin. However, we do not have data to support the idea that hypoalbuminemia from any cause is invariably associated with low SPI. Yet, it seems that in many illnesses, especially if severe, chronic and associated with hypoalbuminemia, the concentration of the SPI decreases. It is not entirely clear what this phenomenon signifies in terms of the physiology of the thyroid hormone, but it emphasizes that caution must be exercised in the interpretation of low values for SPI.

Spectrophotometric Determination of Streptolysin-O Activity and Inhibition. HARRY A. Feldman * and ANNe L. Gillen, Syracuse, N. Y.

Herbert in 1941 reported that the activity of streptolysin- $\mathrm{O}$ and the concentration of antistreptolysin- $\mathrm{O}$ could be measured with greater accuracy if $50 \%$ rather than $100 \%$ end-points were used and that saline phosphate buffered at $\mathrm{pH} 6.5$ and rabbit erythrocytes in approximately $2.5 \%$ concentration were advantageous. Liao later described a modification of the antistreptolysin- $\mathrm{O}$ test using $50 \%$ end-points and rabbit erythrocytes in 5\% concentration. Since the $50 \%$ unit should be superior to the currently favored $100 \%$, this study of the conditions required to define a reproducible $\mathrm{SL}_{\text {nso }}$ unit was undertaken. Sheep erythrocytes stored in modified Alsever's solution were used and initially veronal bicarbonate buffered saline (VBBS) at $\mathrm{pH}$ 7.3-7.4 was the diluent; all readings were made with the Coleman Junior spectrophotometer at wavelength $550 \mathrm{~m} \mu$ and plotted by reference to the Von Krogh equation. It was demonstrated that phosphate buffered saline was preferable and that pH 7.17.2 was superior to 6.5 . With the VBBS, streptolysin-O activity was significantly reduced during incubation but this could be prevented by including glycerine in the mixture or by using phosphate buffer. The $\mathrm{SL}_{\text {Hs }}$ of different lots of streptolysin-O determined in this way did not vary significantly. In contrast to other reports, the relationship between the $\mathrm{SL}_{\mathrm{H} \mathrm{s}_{0}}$ unit and the concentration of erythrocytes is not linear except at erythrocyte concentrations above $3 \%$. In the range $1-3 \%$, the $S L_{\text {Fso }}$ unit is relatively constant. Sheep erythrocytes can be substituted satisfactorily for human erythrocytes if allowance is made for the difference in erythrocyte size. The former are approximately $64 \%$ as large as human erythrocytes; thus a $4.8 \%$ suspension of sheep erythrocytes is numerically equivalent to a $7.6 \%$ suspension of human cells. From these findings a test for the measurement of antistreptolysin- $\mathrm{O}$ which permits for greater reproducibility and for the interchange of erythrocytes of different species has been constructed.

Studies in Parahemophilia. JoHN B. FreLd and ARNOLd G. Ware, Los Angeles, Calif. (Introduced by Paul Starr).

The clinical state of plasma deficiency of accelerator globulin (Ac-Globulin, Proaccelerin, Labile Factor) may be associated with a bleeding dyscrasia. This disease has beed named parahemophilia. The number of such cases which have been studied to date are relatively few, probably numbering less than ten.

Under observation is a large, three generation family group of which nine individuals have significant reductions in plasma accelerator globulin content. Six of these individuals have levels of $50 \%$ of normal or lower. Five others who were found to have normal accelerator globulin also present a history of numerous episodes of epistaxis, easy bruising, impairment of clotting with minor cuts and severe hemorrhage with surgical procedures. Both sexes exhibit the clotting defects. The adult females also have menorrhagia and hemorrhage with parturition. At least ten other members of the family who have not been studied, have a similar history.

The most seriously ill is an eighteen year old female, the issue of two first cousins. She has had the typical subjective clotting impairment from youth with numerous severe bleeding episodes. Since the age of sixteen on, she has had several hospital admissions for long periods. She was studied in detail. Her Ac-G on numerous occasions was zero while her prothrombin concentration ranged from 120 to 140 per cent of normal. Fibrinogen was normal, prothrombin consumption was negligible. Her plasma incubated with normal plasma did not progressively reduce the average Ac-G value of the pooled sample. Given transfusions of fresh plasma, her clotting time was shortened and dental extraction was made possible. The resulting elevation of Acceleration globulin to $5 \%$ of normal was temporary and dissipated to zero within six days. Methylxanthines orally and parenterally, ACTH intravenously and vitamin $\mathrm{K}$ in large doses intravenously were without effect in increasing Ac-G levels.

\section{Prolongation of Viability of the Stored Erythrocyte.} Clement A. Finch * and Beveriy W. Gabrio, Seattle, Wash.

The "storage lesion" of the erythrocyte may be defined as a composite of chemical, physical, and morphologic changes which proceed to ultimate loss of cell viability when blood is preserved in vitro. Because of the importance of carbohydrate metabolism in the maintenance of the erythrocyte our studies have employed the phosphate partition (adenosine triphosphate (ATP), hexosephosphates, 2,3-diphosphoglycerate, etc.) of the erythrocyte 
as an indicator of the metabolic potential of the cell. Post-transfusion survival has been measured employing $\mathrm{Cr}^{\mathrm{st}}$ tagging of stored erythrocytes.

Previous work from this laboratory has shown that the storage lesion is reversible to a point once stored cells are replaced in active circulation. An investigation of systems which cause such a reversal in vitro indicate that the ATP derivative, adenosine, is the most efficient substance in this respect. Following its addition to stored blood, there is: (1) a reconstitution of the depleted cellular organic phosphates, particularly ATP and 2,3-diphosphoglycerate; (2) associated improvements in osmotic fragility, glucose utilization, potassium: sodium ratio, and spontaneous hemolysis. Survival measurements, employing both rabbit and human erythrocytes, indicate better than $70 \%$ viability of the cells preserved in ACD + adenosine at $4^{\circ}$ for periods of 5 to 12 weeks, while control studies show a comparable survival for only 3 to 4 weeks with blood preserved in ACD alone at the same temperature.

These studies demonstrate that membrane integrity and viability are dependent upon the maintenance of erythrocyte metabolism, and that adenosine provides important support to red cell metabolism during storage.

The Effect of Intravenous Hydrocortisone and Corticosterone on the Diurnal Rhythm in Renal Function and Electrolyte Equilibria in Normal and Addisonian Subjects. John T. Finkenstaed, Joseph F. Dingman, Dalton Jenkins, John C. Laidlaw and John P. MerRILL,* Boston, Mass.

Nine studies on two normal subjects and two Addisonians on DOCA were performed. During control experiments, the normal subject showed diurnal rhythm in $C_{12}, C_{p a n}$, and adrenocortical hormone excretion. The Addisonian manifested depressed renal function without nocturnal decline. With 24-hour administration of hydrocortisone ( $1 \mathrm{mg} . / \mathrm{hr}$.) to the Addisonian, there was a sustained increase in renal dynamics. Slightly greater effects occurred with $10 \mathrm{mg} . / \mathrm{hr}$. The normal subject on the larger dose displayed a greater increase in renal dynamics, but diurnal variation persisted.

In control studies on normal and Addisonian subjects, electrolyte $(\mathrm{Na}, \mathrm{K}, \mathrm{Cl})$ excretion was related to changes in electrolyte filtration rather than reabsorption. In the Addisonian, a greatly depressed electrolyte filtration produced a decreased excretion. The normal diurnal variation was absent. In the Addisonian on maintenance doses of hydrocortisone, a sustained elevation of filtration produced increased electrolyte excretion which persisted overnight. With larger doses of hydrocortisone, slightly greater increases in filtration produced an initial electrolyte diuresis. A subsequent increase in reabsorption exceeding filtration occurred. Marked $\mathrm{NaCl}$ retention resulted. With large doses of hydrocortisone in the normal subject, an early electrolyte and water diuresis occurred. Decreased tubular reabsorption, however, was responsible for this negative balance. As in the Addisonian, a delayed but less marked rise in reabsorption produced moderate $\mathrm{NaCl}$ retention. Persistent potassium diuresis was observed on large doses of hydrocortisone.

Corticosterone produced less pronounced changes in renal function and $\mathrm{NaCl}$ equilibria, and a more pronounced potassium diuresis.

The absence of diurnal rhythm in renal dynamics in DOCA-treated Addisonians may be due to the persistent action of this steroid or to pure 11-oxygenated corticoid deficiency. The greater effect of larger doses of hydrocortisone on $\mathrm{NaCl}$ reabsorption in these Addisonians may result from additional DOCA effects or to intrinsic compensatory mechanisms for salt conservation in adrenal insufficiency.

Cerebral Hemodynamics in Acute Hypotension. Frank A. Finnerty, JR., Lloyd Witkin and Joseph F. Fazeras, Washington, D. C. (Introduced by Harold Jeghers).

In an attempt to establish quantitatively the critical rate of cerebral blood flow at which signs of cerebral hypoxia developed, 46 patients were studied including young and old normotensives, hypertensives, benign and malignant, and patients with spontaneous postural hypotension. Cerebral hypoxia was induced by lowering the arterial pressure acutely with intravenous hexamethonium and/or by head-up tilting.

Despite the patient's age, original level of arterial pressure, or original rate of cerebral blood flow, signs of cerebral hypoxia developed when the cerebral blood flow was reduced to $31.5 \mathrm{cc} . / \mathrm{min} . / 100 \mathrm{gm}$. of brain (standard error 0.98 ) and the oxygen delivery to the brain fell to 5 (standard error 0.10 ). The level of arterial pressure at which signs of cerebral hypoxia became manifest varied between 100.7 to $40.9 \mathrm{~mm}$. $\mathrm{Hg}$.

The cerebral vascular resistance fell significantly in all groups, except in the subjects with spontaneous postural hypotension and malignant hypertension. In these 2 groups cerebral hypoxia developed at comparatively normal levels of arterial pressure. The cerebral vascular resistance was the highest in the malignant hypertensive group.

The cerebral metabolic rate did not change and the cerebral arterial-venous oxygen difference increased in all patients.

Angina and electrocardiographic changes were absent during cerebral hypoxia.

Our data demonstrate that there is a critical rate of cerebral blood flow and oxygen delivery to the brain at which signs of cerebral hypoxia become manifest and suggest that the brain is more sensitive to hypoxia than the heart.

\section{Inulin and NA Para-Amino-Hippurate Clearances, Sodium and $\mathrm{H}_{2} \mathrm{O}$ Excretion in Supine Exercising Cardiac $\mathrm{Pa}$ - tients. Oscar W. Freeman, Joseph S. Wirson and A. J. Merrml,* Atlanta, Ga. \\ Other investigators have found consistently decreased $\mathrm{Na}$ excretion in normal and cardiac subjects during ex-}


ercise, irrespective of GFR changes. Twenty four hour $\mathrm{Na}$ excretion studies in this laboratory, with and without exercise, cast some doubt on these findings.

The present studies, done in supine position to eliminate postural effect, include 10 cardiac subjects on digitalis and diuretics. Patients were urged to exercise consistently and strenuously for a 15 minute period. Two types of responses were noted. In 4 compensated and clinically milder cardiacs, no consistent change or correlation of GFR and $\mathrm{Na}$ excretion occurred. In 6 more severe cardiacs GFR consistently fell below $70 \mathrm{cc} /$ minute during exercise. Without exception, GFR averaged $98.7 \%$ and $\mathrm{Na}$ excretion $80.7 \%$ of control in milder cardiacs, with corresponding decrease in RPF. Urine volume changes independently of GFR and $\mathrm{Na}$ excretion. In recovery periods, $\mathrm{Na}$ excretion tended to lag behind GFR in its return toward control values.

$\mathrm{Na}$ excretion is influenced by several factors and during control periods it varied irrespective of GFR. During exercise periods, however, when GFR fell significantly, $\mathrm{Na}$ excretion always showed a comparable diminution. Fifteen normals responded with no consistent change or correlation of GFR and $\mathrm{Na}$ excretion, as did compensated cardiacs.

These findings suggest that the results of others may have been due to posture.

\section{Pentapyrrolidinium $(M \& B 2050$ ) in the Treatment of} Severe Hypertension. EdWard D. FreIs,* EdWard A. Partenope and Lawrence S. Lilienfield, Washington, D. C.

Comparisons were made in the same, previously untreated, hospitalized patients between the ganglionic "blocking" agent pentapyrrolidinium (M \& B 2050) and hexamethonium. The effective dose of pentapyrrolidinium was approximately $1 / 5$ th that of hexamethonium and the duration of hypotensive response lasted $\mathbf{4 0}$ per cent longer. On continuous administration there was less development of "tolerance" and less constipation than with hexamethonium. There also was little cross tolerance; patients on long term hexamethonium treatment, who had become resistant to the hypotensive effects of that drug requiring increased dosages, responded to doses of $\mathrm{M} \& \mathrm{~B} 2050$ which were as little as $1 / 10$ th to $1 / 20$ th the previously required amount of hexamethonium.

Although oral absorption of M \& B 2050 was poor similar to hexamethonium the hypotensive response following oral administration was more uniform and predictable. Constipation was less severe and when present usually could be controlled with oral neostigmine. Ileus was not seen. The other side effects of ganglionic blockade were similar to those observed with hexamethonium. Twentyseven patients have been treated for periods of 5 to 8 months on M \& B 2050 alone. Twenty-two of these have been controlled without severe reactions although dosage adjustment was critical. A few have required parenteral therapy.
In another group of patients treated with M \& B 2050 the addition of reserpine produced further hypotensive effects in approximately half. 1-Hydrazinophthalazine was found to produce additive effects in a similar percentage. Thirty-two patients have been treated for 5 to 8 months with combinations of oral M \& B 2050, reserpine and 1-hydrazinophthalazine. Withdrawal of the latter 2 agents resulted in some increase of average blood pressures in approximately half of the patients.

It is concluded that $M$ \& B 2050, although still producing many of the side effects of ganglionic "blockade" has the following advantages over hexamethonium: (1) smaller dosage requirement, (2) longer duration of hypotensive effect permitting less frequent administration, (3) more predictable response to oral therapy, (4) less gastrointestinal atony and (5) less development of "tolerance."

A Study of the Blood Volume in Congestive Heart Failure. Robert K. Funkhouser and Walter H. PritchARD,* Cleveland, $O$.

Recent studies of cardiac failure using labelled red cells have failed to demonstrate a significant elevation in blood volume, a result which is at variance with the findings of previous investigators using labelled albumin. This study was undertaken to verify both of these findings and if possible to interpret their implications.

Twenty hospitalized patients were selected; eleven controls and nine patients with varying degrees of cardiac edema. In each patient, red cell and blood volumes were estimated by the $\mathrm{Cr}^{31}$-labelled erythrocyte method of Gray and Sterling. Venous samples were drawn over a period of one hour, and immediately thereafter, an injection of $I^{21}$ human serum albumin was given in order to estimate plasma and blood volumes. Hematocrit of each blood sample assayed for radioactivity was determined.

Statistical analysis of the relationship of red cell, plasma, and total blood volume to height, "wet" weight, and surface area was carried out for erythrocyte and albumin dilutions. Results for weight and surface area showed qualitatively similar but quantitatively different relationships to each measured blood volume. The cardiacs demonstrated the following statistically significant differences from the control group:

(1) By albumin dilution, the plasma and blood volumes were much greater.

(2) By erythrocyte dilution, red cell masses were elevated; total blood volume was also elevated even when differences in physical dimensions were considered, but to a lesser extent than by albumin dilution.

This discrepancy in the extent of the difference can be explained on the basis of either of two hypotheses advanced in the past by others: (1) an altered distribution of red cells in the circulation in congestive failure, or (2) the presence in congestive failure of a larger than normal, rapidly equilibrating, extravascular space of albumin distribution. 
The Influence of Androgen and Estrogen on the Association of Lipids with Serum Globulins; Ultracentrifugal and Electrophoretic Studies of Problems in Atherosclerosis. Robert H. Furman, R. Palmer howard and Loyal L. Conrad, Oklahoma City, Okla. (Introduced by Robert H. Bayley).

This study was supported in part by research grants from the National Heart Institute (H-1429) and the Smith, Kline and French Research Foundation.

Estrogens have tended to become associated with prophylaxis against, or delay in the genesis of atherosclerosis, both in chicks (Katz et al.) and human (Barr et al.). The latter note the greater proportion of cholesterol extractable from serum alpha globulin fractions in the female and suggest that this alpha lipoprotein-sex relationship may be the factor responsible for the relative immunity to coronary atherosclerosis enjoyed by premenopausal women. Lewis, however, has demonstrated that alpha-1 lipoproteins, while more concentrated in women than men, continue to increase in concentration beyond the menopause, a time when the female's "immunity" is lost.

Androgen, especially methyl testosterone, and estrogen, chiefly conjugated equine estrogens were studied in relation to their effects on serum lipids and lipoproteins (the latter by means of the analytical ultracentrifuge utilizing a solvent density of 1.21 as described by Lewis) and the electrophoretic distribution of serum proteins.

A variety of human subjects including eunuchoidal males, postmenopausal females, and patients with pituitary dysfunction were studied. Hypogonadism in either sex is associated with increased alpha-1 lipoprotein. Androgens (especially methyl testosterone) are consistently effective in reducing or eliminating alpha-1 lipoprotein. Premarin failed to block this effect. Alpha-2 and beta lipoproteins responded less uninformly. Alpha-2 lipoprotein tended to decrease and to become ultracentrifugally distinct from beta lipoprotein as alpha-1 lipoprotein increased. Diethyl stilbestrol markedly increased alpha-1 lipoprotein in the normally endrogenic male. Serum cholesterol and phospholipid relationships during steroid therapy failed to reveal changes suggesting higher phospholipid concentrations in alpha-1 lipoproteins.

Conventional electrophoresis showed no steroid-induced changes indicative of the marked lipoprotein changes noted with the ultracentrifuge.

It is concluded that the gonadal steroids alter serum lipoproteins by influencing the amount and/or nature of lipids associating with globulins. The hypogonadal state appears to be associated with higher alpha-1 lipoprotein concentrations in both sexes.

Respiratory and Renal Adjustments to Alterations in AcidBase Balance Induced by Diamox, 2-Acetylamino-1,3,4Thiadiasole-5-Sulfonamide, in Normals and in Patients with Chronic Lung Disease. Morton Galdston," New York, N. Y.

Recent studies have demonstrated a linear relationship between renal tubular reabsorptive capacity for bicarbon- ate bound base ( $\mathrm{H}-\mathrm{Na}$ transfer) facilitated by carbonic anhydrase and acutely induced respiratory variations in arterial $\mathrm{pCO}_{2}$ in the regulation of acid-base balance. It seemed important to compare the respiratory system of individuals without heart, lung or kidney disease with individuals with advanced pulmonary emphysema and elevated arterial $\mathrm{pCO}_{2}$ with regard to sensitivity to depletion of serum bicarbonate (metabolic acidosis). This was brought about by ingestion of Diamox, a potent renal carbonic anhydrase inhibitor, in doses of 2.2 to $6.3 \mathrm{mgm} /$ kg. every 6 hours for 1 to 3 months.

Though members of both groups of patients often exhibited approximately the same degree of Diamox induced decrease in serum $\mathrm{BHCO}_{2}(6.0$ to $8.0 \mathrm{mEg} / \mathrm{L})$, their respiratory response to the resulting calculated fall in arterial pH (7.18-7.26) varied considerably. Normals consistently responded with more effective alveolar ventilation, resulting in decreased arterial $\mathrm{pCO}_{2}$. This caused further inhibition of renal tubular reabsorption of bicarbonate bound base to decrease serum $\mathrm{BHCO}_{3}$ an additional $2 \mathrm{mEq} / \mathrm{L}$. As a result, these individuals were able to maintain a slightly lowered arterial $\mathrm{pH}$ of $\mathbf{7 . 3 0}$ to 7.35. In contrast, the abnormals exhibited either no respiratory response or one which resulted in at most an additional fall of $1 \mathrm{mEq} / \mathrm{L}$ serum $B H C O$. Their respiratory acidosis therefore became more intense and occasionally was complicated by metabolic acidosis. The potential handicap of such bicarbonate bound base depletion without adequate respiratory compensation is of considerable clinical importance where further carbon dioxide retention and/or serum $\mathrm{BHCO}$, depletion is likely to occur.

Diamox did not induce any specific alterations in minute volume of respiration and gas exchange. Diuresis was evident only during the first few days of administration.

\section{The Study of Human Serum Phosphatide Patterns in Normal and in Pathologic States as Studied by Quanti- tative Paper Chromatographic Methods. Menard M. Gertler and JACOB KreaM, New York, N. Y. (Intro- duced by Bernard S. Oppenheimer).}

Much attention has been given in the past few years to the role of serum phosphatides in atherosclerogenesis. Since this lipid fraction is heterogeneous it would be desirable and of interest to distinguish the differential changes, if any, in the individual phosphatide components. Quantitative paper chromatographic methods offered interesting possibilities in that they could be adapted, both to the quantitative and qualitative microanalysis of serum phosphatide components such as choline, serine and ethanolamine containing types. Such methods have been developed in our laboratory during the past year permitting direct phosphatide analysis on small samples of serum. Basically the method consists of (a) extraction of the phosphatide components from serum, (b) hydrolysis of the phosphatide extracts, and (c) separation of the nitrogenous components (choline, serine and ethanolamine) of 
the resulting hydrolysate by means of paper partition chromatography and their quantitative estimation by means of quantitative colorimetry.

Data will be presented from (a) 22 normal males of various ages, (b) 32 individuals who have experienced myocardial infarction, (c) 5 pregnant women at various stages of gestation, and (d) 25 samples of serum obtained from cord blood. In addition, data will be presented showing the differential changes observed in the total serum phosphatides and the serum phosphatide components during the administration of various oestrogenic materials to (a) males with coronary heart disease and (b) males with carcinoma of the prostate.

Much information concerning the serum phosphatide pattern has already been accumulated as a result of this investigation. Phosphatides containing choline (e.g. lecithin and sphingomyelin) invariably appear to be the predominant type (60-100 per cent) found in human blood serum. Practically all the samples analyzed, however, do contain appreciable amounts of phosphatidyl ethanolamine and phosphatidyl serine indicting that the so-called cephalin fraction is heterogeneous. The ratio of phosphatidyl serine to phosphatidyl ethanolamine is variable. In certain samples serine appears to predominate over ethanolamine while in others this situation is reversed.

Coagulation Defect and Hemorrhagic Diathesis Due to an Acquired Inhibitor of Antihemophilic Factor. ROBERT Goldstern, Boston, Mass. (Introduced by Samuel L. Gargill).

A circulating inhibitor of antihemophilic globulin has been disclosed as the cause of a recently developed bleeding diathesis in a 40 year old male with a negative family and past history. The disorder was characterized by easy bruising, spontaneous hematuria, hematomata, and hemarthroses. Extensive routine clinical and laboratory investigation revealed no other abnormalities or diseases including disseminated lupus.

Coagulation studies revealed: normal bleeding time; prolonged clotting time (30-120 minutes) ; extremely retarded prothrombin consumption; normal platelets; normal prothrombin time; normal plasma prothrombin, Acglobulin, pro-Spca, fibrinogen, and anti-thrombin activity; questionably significant increase in plasma anti-thromboplastin (human brain). The defective prothrombin conversion was as readily rectified by small amounts of thromboplastin as that of a known hemophiliac, but not by fresh normal blood or plasma. Moreover, as little as one part of the patient's plasma to nine parts of freshly shed normal blood caused retarded consumption in the mixture. By utilizing the prothrombin consumption test to determine the antihemophilic, PTC and PTA activity of plasma and plasma mixtures, the site of action of the anticoagulant could be delineated. The patient's plasma did not correct the retarded prothrombin consumption in hemophilia, but could correct that of PTC and PTA deficiency. Admixture of patient's plasma or serum with normal plasma markedly reduced the antihemophilic activity of normal plasma but left its PTC and PTA activities intact, thus indicating the specific inhibition of the antihemophilic factor. This inhibitor was not adsorbable by $\mathrm{BaSO}_{4}$ and survived heating at $56^{\circ} \mathrm{C}$. for $30 \mathrm{~min}$ utes. Striking similarities between the clotting abnormality of this subject and that seen in resistant hemophiliacs were noted.

The etiology remains obscure. There had been no blood or plasma transfusions before onset of illness. A history of alcoholism and exposure to naphtha and gasoline suggest the possibility of a toxic reaction. Cortisone therapy was ineffective.

The Role of the Adrenals in the Response to Endotoxin. Robert A. Good and Lewis Thomas,* Minneapolis, Minn.

Increased vulnerability to the lethal effect of gram negative bacterial endotoxins occurs in adrenalectomized animals, and this has been interpreted to indicate that resistance to endotoxin involves adrenal function.

The manifestations of intoxication by endotoxins in normal animals include fever, leucopenia, prostration, and the local and generalized Shwartzman reactions. When repeated injections of small doses are given, a state of refractoriness develops, during which none of these responses can be elicited.

It is known that the enhanced susceptibility of adrenalectomized animals is prevented by cortisone. Moreover, cortisone has been found to protect normal adult rabbits against the prostrating and lethal effects of meningococcal endotoxin. On the other hand, no protection against the local or generalized Shwartzman reactions occurs; instead, the reactions are more readily produced in cortisonetreated animals. Furthermore, daily injections of cortisone prevent the development of the refractory state.

In view of the paradoxical differences between the effects of cortisone on different toxic manifestations, the responses to endotoxin in adrenalectomized rabbits were studied.

Typical local Shwartzman reactions occurred in each of 12 adrenalectomized rabbits. The generalized reaction, with renal cortical necrosis, was produced in 3 .

Normal rabbits were given repeated injections of meningococcal endotoxin until they were completely refractory to fever, leucopenia, prostration, and the Shwartzman reaction. At this time both adrenals were removed. Four days later, the degree of resistance was found to be unchanged. The refractory state was also produced in rabbits which had been adrenalectomized before any exposure to endotoxin.

It is concluded that 1) although the adrenals may be implicated in some of the reactions to endotoxin, they are not directly involved in the Shwartzman reaction, and 2) the development of refractoriness to endotoxin is not a function of the adrenals. 
Tubular Reabsorption of Protein in Experimental Nephritis. HowARD GOODMAN and JAMES BAXTER,* Bethesda, Md.

With acceptance of the concept of a normal glomerular filtration and tubular reabsorption of protein, the question arises whether there is an increase or a decrease in the tubular reabsorption of protein in instances of proteinuria. The dye T-1824 has been widely used as a tag for serum proteins, and specifically in studies of the renal clearance of albumin (Chinard, Lauson and Eder, J.C.I. 31, 895, 1952). In addition, the accumulation of the blue dye in droplets in the proximal convoluted tubule cells has been described as a measure of the tubular reabsorption of protein in normal and renin-injected rats (Sellers, Smith, Narmorston and Goodman, J. Exp. Med. 96, 643, 1952). In the present experiments, $2.5 \mathrm{mgm}$. of T-1824 is injected intravenously into normal rats and rats with proteinuria due to anti-kidney serum nephritis and uranium nephritis. With tubular injury due to the administration of uranium, there is less than normal accumulation of the blue dye in the renal tubules. In rats with the nephrotic syndrome produced by nephrotoxic serum, the cortical kidney tubules appear intensely blue due to the accumulation of dye droplets in the tubule cells.

These results suggest that tubular reabsorption of protein is impaired in rats with proteinuria due to uranium nephritis. In proteinuria due to the administration of nephrotoxic serum however, the tubules are reabsorbing greater than normal amounts of protein, and in this instance the proteinuria would seem to be due solely to an increase in glomerular permeability to protein.

Observations on the Hyponatremia Following Mitral Valvulotomy. ALLAN V. N. GoopYrR * and WIILIAM W. L. GlenN, New Haven, Conn.

Twenty-seven patients subjected to mitral valvulotomy were studied before and after operation with regard to the incidence, extent, and mechanism of post-operative hyponatremia. Balances of water, sodium, and chloride were carried out on seventeen patients. The serum sodium fell below 131 meq./1. in thirteen cases in the immediate post-operative period, the serum chloride fell below 99 meq./1. in all cases but one and below 90 meq./1. in thirteen cases. The small negative balances of sodium and chloride were inadequate, by themselves, to account for the observed hyponatremia in all but three cases. Intracellular shifts of sodium (using the chloride space as a reference) were appreciable (over 100 meg.) in only five patients.

The retention of water (allowing for insensible losses) was enough to explain the observed hyponatremia in all but three cases, although the level of fluid intake was not well correlated with the occurrence of hyponatremia.

These results indicate the presence of an unusually prolonged antidiuretic stimulus in these patients, the etiology of which remains uncertain. Except in a few cases it is unnecessary to correct the hyponatremia with hypertonic salt, since it is usually a transient, limited, and essentially asymptomatic phenomenon.

Quantitative Studies of the Circulation in Aortic Valvular Disease. Richard Goritn, Ian K. R. McMintan, Michael B. Matthews, Raymond Daley and W. E. Medd, Boston, Mass. (Introduced by Eugene C. Eppinger).

No data are available concerning left ventricular (LV) systolic pressure, regurgitation volume, LV stroke work or pressure-flow relationships in aortic valvular disease because the first two values, necessary in calculation, cannot be measured directly.

In 1952, McMillan described a cyclic pump wherein aortic valves could be perfused with water under simulated in vivo conditions and motion pictures taken of the functioning valve. The hearts of five patients were so studied at post mortem. From the films stenotic and regurgitant valve areas were measured planimetrically. Utilizing orifice formulae relating orifice area, pressure and flow, with data obtained at prior cardiac catheterization, regurgitant volume and LV systolic pressure were estimated. Total cardiac output (systemic plus regurgitant) and LV stroke work were calculated.

In pure stenosis $\left(0.4 \mathrm{~cm}^{2}\right.$ area; normal $\left.4 \mathrm{~cm}^{2}\right) \mathrm{LV}$ systolic pressure and work were twice normal. In similar stenosis $\left(0.4 \mathrm{~cm}^{2}\right)$ with insufficiency $\left(0.3 \mathrm{~cm} .^{2}\right.$ area), regurgitant volume was one-half systemic, LV systolic pressure twice and work thrice normal. In similar stenosis $\left(0.4 \mathrm{~cm}^{2}\right)$ with tight mitral stenosis, however, because of the very low cardiac output, LV systolic pressure was only slightly increased and work was below normal. In less severe stenosis $\left(0.7 \mathrm{~cm} .{ }^{2}\right)$ with insufficiency $\left(0.4 \mathrm{~cm} .{ }^{3}\right)$ regurgitant volume equalled systemic, LV systolic pressure was two and work four times normal.

Remarkably constant LV systolic pressures between $200-250 \mathrm{~mm}$. Hg were found at rest and on effort. Accordingly, aortic valve gradient and flow had an upper limit, thus fixing stroke output for each patient. This appeared related to the orifice phenomenon; as orifice decreased or flow increased (regurgitation) the exponential pressure-flow curve became flat so that further increases in pressure effected virtually no increases in flow. Critical orifice area for pure stenosis was $0.5 \mathrm{~cm}^{2}$, but was correspondingly larger if there was associated insufficiency.

\section{Brachial Arterial Pulse Wave Contours in Valvular Heart} Disease. David G. Greene and Charles E. Mclean, Buffalo, N. Y. (Introduced by John H. Talbott).

Photokymographic records of intra-arterial pulse pressures have been made at a paper speed of $120 \mathrm{~mm}$ per second with a high frequency blood pressure recording system. Analysis of the resultant form shows several variations among the normal subjects studied, including smoothly rounded peaks of pressure during systole, double 
peaks, and notches or slurs on both the anacrotic and catacrotic limbs. Although the ascent from the diastolic level to the peak of systole is usually a smooth continuous curve with no sudden changes in slope, in three out of 26 normal subjects there was an anacrotic notch. Such a notch was seen more frequently in patients with various lesions of the cardiovascular system. Two out of four patients with pure aortic stenosis demonstrated such a notch. Six out of nineteen patients with mitral valvular disease and clinically normal aortic valves also showed an anacrotic notch. It was seen in all seven patients studied who had aortic stenosis combined with mitral valvular disease. It was noted in two of seven patients with aortic insufficiency. In the normal subjects the anacrotic notch appeared more than 70 per cent of the way up the ascending limb of systole, and the same high position of the anacrotic shoulder was noted in the patients with mitral disease. Patients with pure aortic stenosis, or with aortic stenosis combined with mitral valvular disease, had a lower position of the anacrotic notch. Three of the seven patients with aortic insufficiency showed obliteration of the dicrotic notch, so that the catacrotic limb was a smooth unbroken fall.

The Effect of Chelating Agents Upon the Metabolism of Stable and Radio-Metals. J. GreenberG, H. E. Hart, R. Lewin, H. Spencer, K. G. Stern and D. Laszlo, New York, N. Y. (Introduced by Louis Leiter).

Chelating agents present a new tool to study deposition and removal of physiologic and foreign metals, stable and radioactive, in man. This report deals with an analysis of the underlying mechanism of these processes, selecting as models two radioactive metals which are known bone seekers. Following the intravenous injection of $\mathrm{La}^{100}$ and of $\mathrm{Y}^{\infty}$ chloride high and sustained plasma levels, high uptake by liver and low urinary excretion were observed. It is assumed that these metals, upon being injected into the blood stream, formed high molecular complexes which were not excreted by the kidneys but were transferred to, and mainly deposited in, the liver. After intravenous injection of $\mathrm{La}^{100}$ ethylenediaminetetraacetic acid, (EDTA), $Y^{\infty 0}$ nitrilotriacetic acid (NTA), $Y^{\infty}$ EDTA and $Y^{\infty 0}$ EDTA in the presence of excess Ca-EDTA, the cumulative urinary excretion ranged from $<5 \%$ to $>85 \%$ respectively. These excretory differences are in agreement with the differences in the binding strengths of these metal chelates. Yttrium injected as EDTA-chelate rapidly disappeared from the blood stream, diffused into the tissues, re-entered the vascular compartment and large amounts were promptly excreted in the urine. Yttrium injected as the NTA-chelate also rapidly disappeared from the blood stream. There was no evidence of re-entry, and there was fixation by various tissues, with areas of concentration in bone, liver, spleen and kidneys. The urinary excretion was minimal. These differences in the metabolism of metal chelates may provide a method to measure the ability of biological systems to dissociate metal chelates.
Remission in Nephrotic Syndrome in Children Receiving ACTH. Lawrence Grarnman and T. S. Danowski, Pittsburgh, $\mathrm{Pa}$.

In attempts to influence underlying disease rather than just deliver edema 30 children with nephrotic syndrome of 22 months duration or less were treated with ACTH, $100 \mathrm{mgm}$ daily for 28 days, alone or with nitrogen mustard, while on penicillin and diets containing $10 \mathrm{mil}-$ liequivalents of sodium and 150 milliequivalents potassium.

In 15 of 19 patients edema was delivered prior to ACTH therapy following dextran ("Plavolex"), polyvinylpyrrolidone, urea or a sodium-free diet. Of the patients in whom edema was present at the time the regimen outlined above was started 11 delivered the excess fluid within two weeks, one at the end of four weeks and two after cessation of ACTH; three required paracentesis.

Though decreases in albuminuria and restoration of serum proteins and cholesterol toward normal began during treatment, maximum improvement was often not recorded until several weeks following treatment. Twentyeight of the 30 children have been followed for 1 to 26 months : $64 \%$ were normal and $28 \%$ greatly improved at the last visit. Relapses, responding to re-treatment, occurred in $21 \%$ after remission lasting 3 to 24 months. There is no evidence that nitrogen mustard improved the immediate or the end results.

Three patients developed hyponatremia; in two this followed paracentesis and reaccumulation of ascites. Two developed convulsions with hypertension and serum sodium concentrations of 112 and 116 milliequivalents per liter; one was left with cerebral atrophy following respiratory failure and anoxia. Temporary mental depression occurred in another patient. Mild upper respiratory infections occurred frequently; one patient developed peritonitis.

At this point the results suggest that the regimen of ACTH-low-sodium-high potassium-penicillin produces a high percentage of remissions in the nephrotic syndrome of children, but that recurrences which respond to retreatment are relatively frequent. The marked sodium restriction may result in sodium deficits and convulsions.

The Location of the Hypothalamic Area Controlling the Secretion of Thyrotropin by the Pituitary. Monte A. GrRer and How ARD ERwIN, Bethesda, Md. (Introduced by Harry Eagle).

Bilateral hypothalamic lesions from anterior commissure to mammillary bodies were produced in 424 young adult female rats. Lesions were $0-4 \mathrm{~mm}$. above the base of the skull and 0-1.5 $\mathrm{mm}$. lateral to the midline. After allowing 1-2 weeks for post-operative recovery, the animals were fed $0.15 \%$ propylthiouracil for $10-14$ days. Average thyroid weight in 18 similarly fed normal controls was increased from $7-8 \mathrm{mg} .100 \mathrm{gm}$. body wt. to $16.3 \pm 1.2 \mathrm{mg}$; ; thyroid cell height was also greatly increased. Of the operated rats, 364 had a normal hypertrophic thyroid response and 8 had only slight thyroid hypertrophy. In 54 , thyroid weight was only $4-8.5 \mathrm{mg}$. and there was no increase in thyroid cell height. In the 
last group, the lesions had either destroyed the paraventricular nuclei or were in the midline between the paraventricular nuclei and the infundibulum. In the latter group there was marked cellular loss or almost total disappearance of the paraventricular nuclei, even though the paraventricular area was not directly damaged. This area was not affected in the 364 animals with normal thyroid response.

Seven of the 8 animals with slight thyroid hypertrophy had lesions which partially but not completely destroyed this region. Supraoptic nuclei were usually normal and their complete destruction did not prevent thyroid hypertrophy. Similarly, lesions directly above, rostral, or lateral to the paraventricular nuclei or its caudally extending path to the pituitary did not interfere with thyroid hypertrophy. These findings strongly indicate that the region of the paraventricular nuclei is intimately concerned with the elaboration of thyrotropin by the pituitary.

Body Fluids and Electrolyte Balance in Myxedema. S. I. Griboft, R. WIEnER, J. EIsenberg and A. InNNaccone, New York, N. Y. (Introduced by L. J. Soffer).

Body fluid distribution was studied in one patient with primary, and one with pituitary myxedema. Both subjects had normal extracellular fluid (thiosulfate) and total body water (antipyrine) spaces before treatment. During the period of thyroid medication both showed temporary expansion of thiosulfate space with subsequent fall toward control levels, while the antipyrine space remained essentially unaltered. Both patients showed weight loss and increased urine volume. The subject with primary myxedema showed negative sodium and chloride balances (previously positive), continued positive potassium balance, and elevated serum mucoprotein and hexosamine during the period of therapy, suggesting breakdown of extracellular myxedematous tissue. Expansion of thiosulfate space in both subjects, without comparable change in total body water, might signify redistribution of body fluid but more probably represents increased extracellular penetrability of thiosulfate ion, with consequent increase in measurable extracellular fluid space.

It was previously found that in normal individuals cortisone causes transient increase in extracellular fluid space without change in body weight. Before thyroid therapy the patient with primary myxedema received cortisone for 14 days (150 mg./day), which produced no significant change in body fluid compartments. After 54 days of thyroid medication the addition of cortisone for 17 days produced increased expansion of thiosulfate space without altering total body water or weight. Sodium and chloride balance remained negative, potassium balance positive, and serum mucoprotein and hexosamine elevated. These findings suggest intensification of thyroid effect following cortisone administration, with further breakdown of myxedematous tissue, and indicate that thyroid hormone is necessary for production of cortisone-induced increase in thiosulfate space.
Studies on the Stimulating and Depressant Effects of Acetylcholine on Neuromuscular Function in Man. David Grob,* Richard J. Johns, Victor A. McKusick and A. McGrher Harvey,* Baltimore, Md.

The response to the intra-arterial injection of acetylcholine ( $\mathrm{ACh}$ ), the chemical mediator of neuromuscular transmission, was studied in 26 normal subjects by recording the stimulating and depressant effects of this compound. Injection of $\mathrm{ACh}$ produced prompt stimulation of muscle fibers, attributable to depolarization of motor end plates, resulting in a visible twitch. This was followed by depression of neuromuscular transmission, evidenced by a decrease in the muscle action potentials in response to maximal motor nerve stimuli and by weakness. The depressant effect of $\mathrm{ACh}$, which was proportional to the logarithm of the amount injected, is attributable to persistent depolarization of motor end plates, rendering them temporarily incapable of activation.

Precise measurement of these effects of $\mathrm{ACh}$ provides a tool for studying the physiology and pharmacology of neuromuscular function, and its alterations in disease. The influence of various types of neuromuscular block on the effects of $\mathrm{ACh}$ was studied. Following injection of d-tubocurarine there was elevation in the threshold to stimulation and depression by ACh. d-Tubocurarine block was reversible by $\mathrm{ACh}$. Following block by decamethonium the effects of ACh were not altered, and the block was not changed by $\mathrm{ACh}$. Following anticholinesterase compounds, such as neostigmine or edrophonium, the effects of ACh were greatly enhanced and prolonged. The degree of block increased with successive nerve stimuli, probably because of accumulation of depressant concentrations of $\mathrm{ACh}$.

Increased reactivity to $\mathrm{ACh}$ and prolongation of its effects were also observed after upper motor neurone lesions. These changes were not due to decreased cholinesterase activity. The most striking increase in reactivity ( 20 fold) occurred in a patient who had recently developed basilar artery thrombosis. Since this patient also had myasthenia gravis, the increased reactivity is of interest in view of the suggestion by some that myasthenia gravis is due to decreased responsiveness to ACh.

Nucleic Acid Metabolism in Chronic Lymphatic Leukemia. Lzonard Hammton, New York, N. Y. (Introduced by Joseph $\mathbf{H}$. Burchenal).

Adenine $-8-\mathrm{C}^{16}$ (200 microcurie, $270 \mathrm{mg}$.) was given intravenously to a patient (age 46) with chronic lymphatic leukemia. Thirty-three collections of lymphocytes have been made at intervals over a period of 300 days. The ribose nucleic acid (RNA) and desoxyribose nucleic acid (DNA) have been separated from the cells. The individual purines from each were separated and their radioactivities determined.

It was found that adenine was incorporated as such into the RNA, and to a smaller extent into the DNA, and that there was an eventual extensive transformation of the adenine into RNA and DNA guanine. Radioactivity 
of RNA adenine appeared to reach a maximum at day 5 ( 560 counts/min./ $\mu$ M.) and in RNA guanine at day 14 (180 counts/min./ $\mu$ M.) ; activity in DNA adenine reached a maximum between days 7 to 11 ( 100 counts/min./ $\mu$ M.). The DNA guanine reached an activity (70 counts/min./ $\mu M)$ equal to that of the DNA adenine on about the 30th day. The activity of RNA adenine and guanine likewise reached approximate equality at $\mathbf{3 0}$ days and the activities of the purines in each nucleic acid decreased in parallel thereafter.

The decline in activity in both RNA and DNA could be divided into two phases-an initial more rapid component of approximately 2 months duration and a residual slow component showing a half-time of the order of 300 days. The decline in activity of the RNA greatly exceeded that of the DNA during the initial period, but during the latter period the declines in activities of the RNA and the DNA were more nearly parallel. The prolonged retention of the isotope incorporated into the purines was such that at approximately 300 days, activity in the DNA was still more than one-third of the peak activity, and was greater than that in the RNA.

These results suggest that: (a) leukemic lymphocytes survive for very much longer periods than have hitherto been suspected, namely apparently for over a year, or (b) that leukemic lymphocytes have a remarkable capacity to re-utilize specifically, and with great efficiency, large fragments of the nucleic acids or nucleoproteins of their progenitors.

The implications of these observations will be discussed with reference to the utilization of various precursors by lymphocytes and other cell types.

Capacity of Seriously Wounded Patients to Produce Antibody. W. Paul Havens, Jr., DoN G. Bock and Inwis Srecel, Philadelphia, $\mathrm{Pa}$.

The capacity for production of antibody by young soldiers with severe inanition and hypoproteinemia associated with poor healing of wounds or neurologic injuries was investigated as one aspect of the metabolism of proteins in such patients. Fifteen Schick-negative patients and eleven Schick-negative healthy controls were inoculated with $50 \mathrm{Lf}$ purified diphtheria toxoid, and the amounts of circulating antitoxin were determined at frequent intervals during the following month. The serum albumin before inoculation ranged from $2.0-4.4 \mathrm{gm} . \%$, averaging $3.3 \mathrm{gm} . \%$, in the patients, while the amounts were within normal limits, averaging $4.3 \mathrm{gm} . \%$, in the controls. There were no significant qualitative or quantitative differences in the patterns of immunologic response in the two groups. The maximum measured amounts of antitoxin produced by the patients averaged 38.85 units/ $\mathrm{ml}$. serum, while the controls averaged 26.50 units $/ \mathrm{ml}$. serum. No apparent relationship existed in either group between the amounts of serum albumin or total proteins present immediately before inoculation with diphtheria toxoid and the amount of circulating antitoxin which subsequently developed. There was also no evident relationship between the capacity to produce antibody and the age of the patient, clinical condition, degree of malnutrition, or resistance to infection. The only significant relationship determined in both groups was between the amounts of circulating antitoxin found before the Schick test and the maximum absolute increases in antitoxin attained after inoculation of toxoid. In general, the greater the amount of pre-Schick circulating antitoxin, the greater was the maximum measured amount subsequently attained.

Cardiocirculatory Performance in Atrial Flutter. RtJaNE M. Harvey, Andre Cournand and M. Irente Ferrer,* New York, N. Y.

A series of nine patients with atrial flutter have been studied using the cardiac catheterization technique and measuring cardiac output (Fick principle), blood volume, right ventricular, pulmonary arterial and peripheral arterial pressures. The basic pressure curves illustrate the constant occurrence of a mechanical auricular activity with each electrical flutter wave. Right ventricular and pulmonary artery curves as well as the atrial curve itself demonstrate the impact of each of these rapid atrial systoles. This is in sharp contrast to the pressure curves in atrial fibrillation where individual atrial systoles cannot always be identified. Furthermore the pressure pattern of tricuspid insufficiency, so commonly observed in atrial fibrillation, rarely occurs in atrial flutter.

A patient without evident heart disease in atrial flutter has been compared to the group with the arrhythmia and a variety of intrinsic heart diseases. In this latter group four patients in congestive failure were acutely digitalized, and two of these were studied again after conversion to normal sinus rhythm. Finally two patients in flutter but not in congestive failure were observed at rest and during exercise and then under these two conditions after conversion to sinus rhythm. From the accumulated data it is evident that this arrhythmia is associated with a low cardiac output only in those subjects with intrinsic heart disease, since the blood flow was normal in the one man without disease. In addition, the resting cardiac output rose after conversion to sinus rhythm in two subjects, which is reminiscent of observations in atrial fibrillation made by other observers. In general, a small pulmonary artery pulse pressure and a low level of pulmonary hypertension were found in those subjects with atrial flutter and congestive failure in contrast to patients in failure with sinus mechanism.

The Fate of Hydrocortisone-4-C $C^{14}$ in Man. LEON HELLman, H. Leon Bradlow, Jerome Adesman, David K. Furushma, J. Laurence Kulp and T. F. Gallaghro, New York, N. Y. (Introduced by C. P. Rhoads).

Hydrocortisone-4- $\mathrm{C}^{2}$ was given intravenously to a series of human subjects in both tracer and therapeutic quantities and the following observations were made concerning the metabolism of this principal adrenal hormone.

1. The routes, rate, and amount of excretion have been defined. $70-80 \%$ of the administered radioactivity was 
eliminated in urine within 24 hours at a rate characterized by a half-life of 3.6 hours. An additional $6 \%$ appeared in urine during the next two days and $9 \%$ was recovered in feces in four days.

2. The absence of hormone breakdown, as indicated by loss of $\mathrm{C}^{14}$ from ring $\mathrm{A}$, was established by the failure to find $\mathrm{C}^{14} \mathrm{O}_{2}$ in expired air by the most sensitive method of radiocarbon analysis.

3. The metabolism of the hormone was independent of the amount administered since the rate and cumulative excretion were unchanged with amounts of hydrocortisone from 0.25 to $100 \mathrm{mg}$.

4. The original hydrocortisone was rapidly subjected to chemical alteration since $85 \%$ of the material excreted in the urine was in a conjugated form within two hours. The concentration of the free hormone and its metabolites in the circulation was measured and, as demonstrated by isotopic dilution, a minimum of $87 \%$ of the circulating radioactivity was present in a form other than the original hydrocortisone within fifteen minutes after infusion.

5. By these criteria, the metabolism of hydrocortisone was shown to be independent of physiologic and pathologic variations induced by disease.

6. The problem of "tissue demand" for hormone, as evidenced by alterations in these processes, was explored in an adrenalectomized oophorectomized patient and no differences were observed in the fate of tracer doses given at a time when all replacement thrapy was withdrawn and again when the tissues of the same subject were saturated with hormone.

Some Metabolic Effects of Glutathione Given Intravenously in Man. Dorothy H. Henneman, Boston, Mass. (Introduced by Mark D. Altschule).

Large doses of glutathione (Schwarz "Triptide") were injected intravenously in three normal men and thirty-one psychotic patients in a total of forty-nine experiments. No toxic reactions occurred if certain precautions were observed.

In the fasting state giving glutathione decreases the total serum inorganic phosphate, whole blood lactic and pyruvic acids, plasma citric acid, and alpha-keto-glutaric acid.

Four and twelve gram doses administered during thirteen oral or intravenous glucose tolerance tests had no effect on the normal glucose tolerance. However, larger doses, i.e. $0.5 \mathrm{gm} / \mathrm{kg}$., increased the normal tolerance of one normal male and changed the abnormal carbohydrate metabolism of one psychotic patient to normal. In four psychotic patients a dose of four grams significantly decreased the lactic acid rise observed after administration of glucose; no change in glucose tolerance occurred with this small dose.

Glutathione in doses of $12 \mathrm{gm}$. and of $0.5 \mathrm{gm} / \mathrm{kg}$. had no effect on the carbohydrate changes induced in the oral or intravenous glucose tolerance by a one to three day course of Compound $F$, or ACTH, in five psychotic patients and one normal subject.

A dose of $0.5 \mathrm{gm} / \mathrm{kg}$. of glutathione increased the reaction to insulin in one psychotic patient. The abnormal carbohydrate response to epinephrine became normal in one psychotic patient after the administration of glutathione.

Depending upon the dose administered, glutathione increased the fall in total serum inorganic phosphate due to glucose, levulose, pure sodium d-lactate, insulin, epinephrine, and the combined action of ACTH and glucose.

The Mechanism Responsible for Hypercalcuria in Sarcoid. Phillp H. Henneman, Evelyn L. Carroll and Eleanor F. Dempsey, Boston, Mass. (Introduced by Fuller Albright).

A male patient with sarcoid was investigated with a complete balance study for calcium, phosphorus, nitrogen, sodium, potassium, and chloride. The study was divided into 6 days fore-control, 30 days oral cortisone acetate therapy, and 12 days after-control.

Initially the patient exhibited hypercalcemia and hyperphosphatemia and markedly negative calcium and phosphorus balances. On a diet containing $292 \mathrm{mg}$. of calcium, the fecal calcium was only $23 \mathrm{mg}$. while the urinary calcium averaged $930 \mathrm{mg}$. per day. Cortisone produced a fall in the serum calcium, a steplike fall in urinary calcium to a minimum of $216 \mathrm{mg}$, and a rise in fecal calcium to a maximum of $417 \mathrm{mg}$. On discontinuing cortisone the urinary calcium had risen to $448 \mathrm{mg}$. per day by the 12th day.

The mechanism responsible for hypercalcemia and hypercalcuria in this patient appears, at least partly, to be an excessive absorption of calcium from the gastrointestinal tract in a pattern suggesting hypervitaminosis $D$. That excessive absorption is not the entire explanation is suggested by a urinary calcium excretion markedly in excess of dietary intake, a relation which could be explained, however, by the second or parathyroid-hormonelike action of vitamin D. These two actions of vitamin D are considered to be:

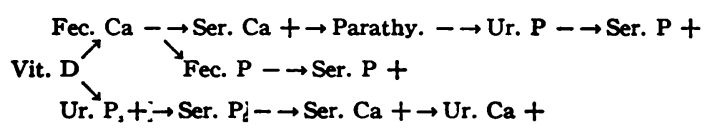

Note is made of the finding of unusually low fecal values for nitrogen, sodium, and potassium, a finding noted previously during overdosage with dihydrotachysterol.

In confirmation of this study data are presented from balance studies on two other patients with sarcoid. In each instance the finding of a very low fecal calcium coupled with a very high urinary calcium supports the suggestion that the mechanism responsible for hypercalcuria in sarcoid may be excessive endogenous production of vitamin D-like-substances.

Evidence of a Regulatory Role of the Thyroid in the Excretion of Nitrogen During Stressful Life Experiences in Man. Basil S. Hetzel, William W. Schottstaedr and William J. Grace, New York, N. Y. (Introduced by Harold G. Wolff).

Serum protein bound iodine (P.B.I.) and urinary excretion of nitrogen, sodium, and potassium before, during, and after experimentally induced stressful life experiences 
have been observed in euthyroid and in hypothyroid subjects maintained on dessicated thyroid.

In 14 studies on 13 euthyroid subjects, mean initial levels were P.B.I. 5.2 gamma per cent, nitrogen excretion $6.4 \mathrm{mg} . / \mathrm{minute}$, sodium $112 \mu \mathrm{Eq} . / \mathrm{minute}$, and potassium $48 \mu \mathrm{Eq}$./minute. During the period of stress (lasting approximately an hour), increases up to 87 per cent (mean 50 per cent) in nitrogen excretion, up to 443 per cent (mean 109 per cent) in sodium excretion, and up to 114 per cent (mean 59 per cent) in potassium excretion were observed. Significant elevation of serum P.B.I. was observed in 7 instances. In 6 such studies on 5 hypothyroid subjects, mean initial levels were P.B.I. 4.6 gamma per cent, nitrogen excretion $4.5 \mathrm{mg} . /$ minute, sodium excretion $87 \mu \mathrm{Eq} . / \mathrm{minute}$, and potassium $37 \mu \mathrm{Eq} . /$ minute. During the period of stress increases up to 49 per cent (mean 21 per cent) in nitrogen excretion, up to 185 per cent (mean 69 per cent) in sodium excretion, and up to 45 per cent (mean 26 per cent) in potassium excretion were observed. Significant elevation of serum P.B.I. was not observed.

The differences in the increases in nitrogen and potassium excretion in the two groups were significant $(P<0.05)$ but the differences in the increases in water and sodium excretion were not $(P>0.05)$.

No differences from euthyroid subjects were observed in one adrenalectomized subject and one Addisonian subject maintained on cortisone and desoxycorticosterone acetate.

The findings suggest that the thyroid is exerting a "regulatory" role in the excretion of nitrogen and potassium during stressful life experiences in contrast to the "permissive" role of the adrenal cortex.

Studies of Pregnancy in Adrenal-Deficient Women. A G. Hills, E. H. Venning, G. W. Webster and E. M. Richardson, Philadelphia, $\mathrm{Pa}$. (Introduced by $\mathrm{F}$. C. Dohan).

The occurrence of pregnancy in two women who because of severe hypertensive disease had been adrenalectomized (subtotally and totally respectively) permitted studies concerning the question of "adrenal-cortical like" activity in the adrenal-deficient pregnant woman. Urinary elimination of neutral 17 -ketosteroids and of neutral reducing lipids (chemocorticoids) was markedly and progressively increased in both women from early in the second trimester until delivery. Biocorticoids (glycogenic corticoids) were excreted in the urine of both women during the latter half of pregnancy in quantities amounting to about four times the values observed during the non-pregnant periods.

The quantity of 17-ketosteroids and of biocorticoids excreted by these women in the latter half of pregnancy was in the general range of values observed at corresponding periods in normal pregnancy, and the biocorticoid excretion was of the order of magnitude encountered in Cushing's syndrome. In spite of these high values for urinary steroid excretion, both women were shown to be vulnerable to the development of acute adrenal insufficiency during the second and third trimesters of preg- nancy; and in the one patient who required steroid therapy at all times, no decrease of hormone requirement accompanied pregnancy.

The conjunction of adrenal insufficiency with the very high values for the urinary steroids, especially the biocorticoids, indicates either 1) that pregnancy greatly increases hormone requirement or 2) that during pregnancy the urinary excretion of chemocorticoids, 17-ketosteroids, and biocorticoids fails to afford a roughly quantitative reflection of available (secreted plus exogenous) cortical hormone, owing to a presumptive alteration of the usual pathways of steroid metabolism resulting in augmentation of the proportion of secreted steroids and their metabolites eliminated in the urine. A definitive choice between these possible explanations is not warranted at present.

Some Aspects of Kidney Function in Hepatolenticular Degeneration (Wilson's Disease). ROBERT E. HodGes, Walter M. Kirkendall, Charles Schwartz and J. Burnetre Wind, Iowa City, Ia. (Introduced by Horace M. Korns).

The cause of increased renal excretion of amino acids and copper in Wilson's disease is unknown. Uzman suggested that aminoaciduria causes increased copper excretion when oligopeptide compounds are in a chelation complex with copper. The aminoaciduria may result from competition between free amino acids and peptides for common tubular transport mechanisms.

We studied four patients with Wilson's disease and three normal persons with standard renal clearance methods using insulin and para-aminohippuric acid (PAH) in protein hydrolysate and determined renal clearances of copper and amino acid nitrogen. Renal extraction of PAH was measured in one patient with Wilson's disease.

Glomerular filtration was within normal limits in both groups of patients. Renal plasma flow in the Wilson's disease patients was approximately $2 / 3$ of the normal. Renal extraction of PAH was 91 per cent in one patient with Wilson's disease. During protein hydrolysate infusions plasma levels of amino acid nitrogen rose and copper remained unchanged in both groups. Clearances of amino acid nitrogen rose equally in both groups. However, clearances of copper rose only in the Wilson's disease patients and averaged about three times pre-infusion rates.

These findings demonstrate a significant reduction in renal blood flow in this group of patients with Wilson's disease. With the normal glomerular filtration this suggests abnormal renal vascular resistance. The parallel rise in excretion of copper and amino acid nitrogen is in keeping with Uzman's hypothesis of competitive tubular reabsorption. The findings might also be explained by postulating a primary tubular defect.

The Role of Regeneration in the Morphogenesis of Experimental Fatty Cirrhosis. F. W. HOFFBAUER,* Minneapolis, Minn.

The transformation of a fatty liver to cirrhosis has been studied in male rats (initial weight 100 grams) fed a 
choline deficient diet. Five recognizable stages are: I, (10-15 days) centrolobular cells fatty. II, (15-30 days) all liver cells fatty, centrolobular fatty cyst disintegration underway. III, (30-60 days) periportal regeneration (normal hyperplasia). IV, (60-90 days) compression of centrolobular fatty cysts and formation of fibrous septa linking central veins. V, (90 days to death, 120-180 days) pathological regeneration-concentric nodular hyperplasia.

The manner of liver regeneration, initially of a normal order (stage III and IV) and later pathological in character (stage V), appears to determine the pattern of architectural alteration.

The situation in stage II is comparable to that of an animal subjected to partial hepatectomy, i.e. there is a marked deficiency in the number of functioning liver cells. The response is regeneration-normal hyperplasia. The multiplication of cells occurs about the small portal triads where the parenchyma is the least fatty. This periportal growth of new cells exerts pressure on the disintegrating fatty cysts (stage III) and, continuing, determines the location of septa composed of micromembranes (stage IV). The normal hyperplasia increases the size of the already large fatty liver. As long as it persists, the liver maintains a characteristic microscopic picture (fibrocirrhosis-normal spatial relationship, lobular pattern outlined by fibrous septa). The animal remains healthy.

There is a constant loss of functional tissue as newly formed cells become fatty. This provides a continuing intense stimulation for regeneration. Ultimately the orderly growth of new cells gives way to pathological regeneration (stage V). Individual concentric hyperplastic nodules appear and gradually increase in number. Normal regeneration apparently ceases and the growing nodules predominate. The microscopic picture (fibronodular cirrhosis) is one of disorganization; distortion and compression of the original liver tissue and especially the intrahepatic veins is apparent. During this stage the size of the liver usually decreases, the animal loses body weight and terminally exhibits effusions.

Effects of $\mathrm{CO}_{2}$ Retention on Intracellular Acid-base Balance. DuncaN A. HolAdaY, New York, N. Y. (Introduced by E. M. Papper).

Blood and thigh muscle samples were obtained from 15 anesthetized dogs before and after 90 minutes of normal respiration or induced hypoventilation with an oxygen enriched gas mixture. These methods of ventilation did not reduce arterial saturation to levels below the control in 14 experiments. $\mathrm{pH}, \mathrm{CO}_{2}$ content, oxygen content and capacity, hematocrit, chloride, sodium, potassium, and water content were obtained on whole blood and plasma; inorganic phosphate and lactic acid contents were obtained on plasma. Tissue chloride, sodium, potassium, water, and fat contents were determined. Tissue $\mathrm{CO}_{2}$ content was analyzed by the method of Danielson and Hastings. Derived values included: venous blood, $\mathrm{pCO}_{2}$, buffer base (Singer and Hastings nomogram), extracellular water and electrolyte contents, intracellular
pH, water and electrolyte contents. Control animals exhibited insignificant changes in the above values. Increases in $\mathrm{pCO}_{2}$ betwen 74 and $138 \mathrm{~mm}$. $\mathrm{Hg}$ maintained for 90 minutes resulted in an average reduction in plasma $\mathrm{pH}$ of 0.55 , and a reduction in buffer base of $15.6 \mathrm{meq} . / \mathrm{L}$. of blood. The mean increase of blood bicarbonate was only $2.8 \mathrm{mM}$./L. Intracellular $\mathrm{pH}$ was reduced to the same extent as the plasma $\mathrm{pH}$. The bicarbonate and sodium and potassium contents of cells remained essentially unchanged. If a buffer capacity similar to that of blood can be ascribed to cellular proteins, then decrease in $\mathrm{pH}$ was probably accompanied by the appearance of significant amounts of intracellular acids, as yet unidentified. These changes are also accompanied by a decrease of salt and water in the blood and an increase of salt and water in cells. Intermediate $\mathrm{pCO}_{2}$ elevations $(+30$ to +57 $\mathrm{mm} . \mathrm{Hg}$ ) result in similar alterations of a proportionally smaller magnitude.

\section{Biochemical Determination of Blood and Urine Catechol Amines as a Measure of Sympathoadrenal Activity in Hypertension. S. W. HoobleR,* A. AGRest and R. J. Warzynski, Ann Arbor, Mich.}

By the method of Weil-Malherbe (Bioch. J. 51 : 311, 1952), it was possible to detect increases in the level of blood catechol amines under conditions of environmental stress in some hypertensive patients and a regular decline in these compounds when sympathoadrenal activity was suppressed by ganglionic blocking drugs. In two patients, stressful interviews raised the level of blood catechol amines from 1.6 to 3.1 and from 4.1 to $5.4 \mathrm{mcg}$./liter respectively.

In all seven patients treated with ganglionic blocking agents, a decline in blood level was noted. The mean value before treatment was $2.7 \mathrm{mcg}$./liter and after treatment was $0.47 \mathrm{mcg}$.

The decline in blood catechols was not proportional to the degree of reduction of blood pressure, and did not regularly occur after supradiaphragmatic sympathectomy $\left(D_{8}-D_{13}\right)$. Twelve normal patients, 2 hyperthyroid, 17 "stable" and 18 labile hypertensive individuals showed no significant difference in resting blood catechol levels.

The method of Weil-Malherbe was modified for urine analyses by removing the catechols on an alumina column at $\mathrm{pH} 8.4$, eluting with $0.2 \mathrm{~N}$ acetic acid, concentrating to dryness and separating from each other by paper chromatography. In 6 normals, the average 24-hr. secretion of noradrenaline was $104.7 \mathrm{mcg} . / 24 \mathrm{hrs}$., adrenaline was $33.2 \mathrm{mcg}$. and hydroxytyramine $87.6 \mathrm{mcg}$. Fourteen hypertensive subjects showed no significant differences.

In one case of pheochromocytoma, the urinary catechols were noradrenaline 2,534, adrenaline 160 and hydroxytyramine 1462 mcg. per $24 \mathrm{hrs}$.

In conclusion there appears to be an approximate correlation between the blood levels of catechol amines and sympathoadrenal activity. On the basis of this measurement, no increase can be noted in resting hypertensive subjects. Their response to stress remains to be studied. 
24-hour urine excretion of the individual catechol amines can now be measured with fair accuracy, and does not appear altered in essential hypertension.

\section{Blood (Red Cell) Volume Measured by Carbon Monoxide} and Radiochromium Methods. JAMES HoPPER, JR., Norman Nomof, Ellen Brown, Reidar Wennesland and Kennetr G. Scotr, San Francisco, Calif. (Introduced by T. L. Althausen).

$\mathrm{CO}$ and $\mathrm{Cr}^{\text {ol }}$-available spaces were determined simultaneously under resting conditions in 6 healthy subjects and 29 patients with various diseases. In all but 2 of the 35 subjects, the CO space was greater than $\mathrm{Cr}^{\text {sil }}$ space, and the average discrepancy was $16 \%$. The difference in spaces was of the same magnitude in disease states as in health. Distribution of both $\mathrm{CO}$ and $\mathrm{Cr}^{\mathrm{sl}}$-tagged cells was complete within 20 minutes, and $\mathrm{Cr}^{51}$-space remained nearly constant for 24 hours. It is concluded that (a) the entire contents of the vascular compartment are reached by these indicators, and (b) $\mathrm{CO}$ is distributed to a space which is about $16 \%$ larger than intravascular red cell volume. Since the CO reaches its maximum volume of distribution during the first 20 minutes of re-breathing, it is assumed that it becomes bound by substances having affinity for CO similar to that of the hemoglobin in circulating cells. Experiments are in progress to determine the areas of the body where such combination occurs. Relative concentration of $\mathrm{CO}$ and $\mathrm{Cr}^{51}$ is determined in various tissues of the rabbit, including bone marrow. Differential measurements of $\mathrm{CO}$ and $\mathrm{Cr}^{51}$ spaces may prove to be a means of estimating stores of non-circulating hemoglobin-like substances.

Clinical and Sub-Clinical Responses to Poliomyelitis Infection in Families. D. M. Horstmann * and R. W. McCollum, New Haven, Conn.

The present study is concerned with factors governing the incidence of infection among familial and other close contacts of poliomyelitis cases. Attention was focussed on the correlation of infection (or its absence) with immune status, and on the relation of age to clinical manifestations of the disease. Such a study is now feasible because of the introduction of tissue culture techniques into poliomyelitis research.

The group studied consisted of 32 index cases and their 91 household and daily contacts. Twenty-eight of the contacts were found to be infected with Type 1 poliomyelitis virus; 13 of these had symptoms compatible with the "minor illness," and 15 were asymptomatic. When the antibody status of these who failed to become infected was determined, it was found that a number already possessed Type 1 antibodies, and could therefore be considered immune. When only susceptibles were considered, the infection rates were very high indeed : of 73 household and daily contacts under 15 years of age, 29 were susceptible; of these, $26(90 \%)$ were infected. In contrast, of the 18 in the age group 15 years old and over, 10 were susceptible, and only 2 of these $(20 \%)$ were excreting virus.
Although infection rates were uniformly high through 0-14 years, the clinical response in different age groups varied. $A$ trend toward milder infections and less paralysis was noted in infants and young children when clinical manifestations of the 60 infected individuals (32 cases and 28 contacts) were analyzed. The ratio of inapparent to apparent infections was about $1: 1$, in contrast to the estimated figures of $100: 1$ for the general population.

Cardiac Output by Body Surface Counting of I's Human Serum Albumin. Rex L. Hurf,* D. D. FelurR and George Bogardus, Seattle, Wash.

A new and simple technique for measuring cardiac output has been devised employing body surface counting following a single intravenous injection of $I^{\text {In }}$ Human Serum Albumin (HSA). The simplicity, reliability, and rapidity of the method give great advantages over other methods. It can be carried out in a physician's office and can be used to measure the cardiac output of exercising individuals.

The injection of $\mathrm{I}^{\text {12 }}$ HSA is made into the antecubital vein of man or the femoral vein of dogs and the counting rate recorded from a narrow-angle scintillation counter placed on the skin between the first and second ribs at the left parasternal line. Count rates as a function of time are obtained which are similar to those obtained by direct arterial blood sampling. The area under the extrapolated curve, measured as in the direct eye or radioisotope procedure, is divided into the equilibrium counting rate. The dimension of the cardiac output thus obtained is in terms of a multiple or fraction of the subject's blood volume per unit time. Conversion to liters/min. is made by taking several venous blood samples for blood volume determination.

Validity and reliability of the technique are affirmed by the fact that measured cardiac indices of hospitalized patients agree with those expected from the history and physical findings of the patient. Normal individuals have normal values. Furthermore, simultaneous cardiac indices measured by the Direct Fick Method agree favorably with this in vivo isotope method.

Up to three separate determinations may be performed at one visit within a half hour period. In some cases, further tests are limited by the radiation tolerance doses for $I^{\text {In }}$; however, repetition is permissible at a later date.

A Study of the Physiologic Closure of the Ductus Arteriosus in Newborn Infants. Herbert N. HuLtGreN, Frederic L. Eldridge and Mary E. Wigmore, San Francisco, Calif. (Introduced by Lowell A. Rantz).

The exact time of functional closure of the ductus arteriosus in the normal human infant has never been determined, although animal experiments have suggested closure immediately after birth.

To determine this, arterialized capillary blood samples were obtained from the right hand and foot of 33 normal 
newborn infants and analyzed for oxygen content and capacity.

31 of the 33 infants showed right hand oxygen saturations of $90 \%$ or more, indicating that true arterial blood was obtained. Up to 3 hours after birth, the oxygen saturation of blood from the foot was significantly lower than that from the hand in 9 of 12 cases studied, indicating the presence of a veno-arterial shunt through the ductus arteriosus. Calculations in these cases indicate the ductus was contributing from to the total blood flow in the descending aorta. In infants 3-72 hours after birth, 7 of 15 cases showed evidence of a veno-arterial shunt through the ductus. None of the 6 infants over 3 days of age showed any significant difference in saturation between right hand and foot.

These findings indicate that the ductus arteriosus is patent, that a veno-arterial shunt occurs in most infants under the age of 3 hours, and that this may persist in a significant number of infants up to the age of 3 days. In those infants showing no difference in hand-foot saturations and in infants over 3 days of age, either the ductus had closed or the shunt had become arterio-venous.

Low oxygen mixtures (12\%) were administered to 4 infants showing no evidence of veno-arterial shunt. Significant differences in hand-foot saturations were produced indicating either re-opening of a closed ductus or reversal of flow through a patent ductus due to increased pulmonary vascular resistance.

The Effect of Cortisone and Hydrocortisone on the Peripheral Utilization of Thyroid Hormone. SIDNEY H. INGBAR and Norbert Freinkel, Boston, Mass. (Introduced by Maxwell Finland).

Changes in iodine metabolism have been described during induced hyperadrenocorticism or stressing environmental manipulations. Studies were designed to investigate the mechanisms whereby these changes are induced.

By a method designed to minimize errors resulting from mixing phenomena, thyroxine utilization rates were determined in 9 patients on constant diets before and during the administration of cortisone or hydrocortisone. Thyroxine space was calculated daily for 8-16 days as the quotient of the residual radiothyroxine (administered dose minus fecal, urinary, and thyroidal collection of radioiodine) and the plasma radiothyroxine concentration. After distribution equilibrium had been achieved, as evidenced by constancy of the calculated space, the fractional daily turnover was determined from the exponential slope of the plasma concentration. Utilization rate was calculated as the product of the plasma PBI and the daily volume turnover (fractional turnover $X$ space).

In 4 patients with primary myxedema and in 3 in whom thyroid function had been abolished by exogenous hormone, alterations in pituitary and thyroidal function were eliminated as experimental variables. Daily doses of 75-200 mg. of steroid in these patients and of $25 \mathrm{mg}$. in 2 patients with panhypopituitarism failed to change utilization rates or PBI's significantly. In the latter pa- tients cortisone did not directly suppress thyroidal function, since calculated values of the daily hormone production were also unchanged.

These data suggest that during periods of dietary control, rates of thyroxine utilization are not conditioned by the availability of 11-17-oxygenated steroids. Hence other mechanisms must be invoked to explain changes in iodine economy which follow certain noxious stimuli.

Bacteriophage Typing of Staphylococci in Clinical Epidemiology with Observations on Antibiotic Resistance. George Gee Jackson and Mark H. Lepper, Chicago, III. (Introduced by Harry F. Dowling).

Clinical investigation of infections caused by staphylococci has been impeded by the lack of a suitable means of classifying strains within the species except by broad biologic characteristics. Using 20 staphylococcic bacteriophages, 2085 strains of coagulase positive and coagulase negative staphylococci isolated from 795 persons have been tested for patterns of lysis. One-hundred-twenty-nine patterns of strong lysis were observed among 541 coagulase positive strains, and these enabled the identification of specific strains with a high probability of validity.

The bacteriophage reactions permitted classification of typeable strains of staphylococci into 4 broad groups according to the grouping of phages suggested by Williams on the basis of the association of lysogenic reactions, and 4 additional intermediate groups. Most of the broad phage groups were represented among cultures taken from different anatomical sites but particular groups showed a statistically significant increase or decrease in the frequency of occurrence in the respiratory tract. Ninety per cent of the strains isolated from patients with staphylococcic enteritis in 3 hospitals could be assigned to 2 phage groups. The distribution of the phage groups among patients, their contacts, and hospital personnel was not in the same proportion as their overall representation. Observations were made on the exchange of strains in persons within each of these population categories.

The development of resistance to the action of penicillin, chlortetracycline and erythromycin does not alter the phage reaction of the strain and statistically significant differences in the number of sensitive and resistant strains were observed among the different phage groups for each of the antibiotics tested.

The Effect of 17 a-Hydroxyprogesterone upon the Urinary Excretion of 17-Ketosteroids and "Pregnanediol Complex" and its Possible Role in Congenital Adrenal Virilism. Joseph W. JaIler, * Jay J. Gold, Seymour Lirberman and Raymond VAN de Wiele, New York, N. Y.

Previous investigations have shown that the administration of ACTH to patients with congenital adrenal virilism results in no increase in the excretion of formaldehydogenic steroids; no retention of sodium; and no fall in eosinophils (Wilkins et al.; Bartter and Albright; Jailer et al.), but does result in a marked rise in the 17ketosteroid and "pregnanediol complex" excretion. Con- 
sequently, one can postulate that the adrenals of these patients have difficulty in synthesizing hydrocortisone, and elaborate instead steroids which are precursors of the 17-ketosteroids and the "pregnanediol complex." Hechter, Pincus, and others have demonstrated that progesterone can be hydroxylated by surviving adrenal tissue at the C-11, 17, 21 positions to form hydrocortisone. One of the intermediary steroids formed in this biosynthesis is $\mathbf{1 7}$ $\alpha$-hydroxyprogesterone. This steroid had been previously isolated from adrenal tissue by North and Pfiffner, and has been shown to be androgenic when administered orally to chicks. 17 $\alpha$-hydroxyprogesterone has been administered orally to 8 patients; 2 patients with Addison's disease, 2 patients with hypopituitarism, and 4 patients with the adrenogenital syndrome whose 17-ketosteroid excretion was being suppressed with cortisone. In all cases a rise in the 17-ketosteroid and "pregnanediol complex" excretions occurred. Chromatographic separation and infra-red identification of the 17-ketosteroid complex revealed the presence of androsterone, etiocholanolone, and 17-hydroxypregnanolone which are urinary metabolites usually found in the adrenogenital syndrome. One can speculate that in adrenal virilism an enzymatic block exists which prevents the complete hydroxylation (probably at C-11 and C-21) of steroidal precursors of hydrocortisone. This may result in the secretion of excessive amounts of 17 $\alpha$-hydroxyprogesterone. Since 11-oxygenated 17-ketosteroids are found in high concentrations in the urine of some patients with virilism, it is possible that the block in hydroxylation in these patients occurs only at the C-21 position, and this may lead to the excessive secretion by the adrenal of " $11 \beta, 17 \alpha$-dihydroxyprogesterone" or 21-desoxyhydrocortisone which then is metabolized to 11-oxygenated ketosteroids.

Studies on the Mechanism of the Anemia of Liver Disease. JAMES H. JANDL, Boston, Mass. (Introduced by W. B. Castle).

Red cell survival studies on 20 patients with chronic alcoholism, hepatic cirrhosis, and macrocytic anemia revealed a hemolytic process proportional in severity to the degree of anemia. The calculated daily hemoglobin production and the total urobilinogen excretion of these patients both averaged 1.5 times normal, and ranged up to 3 times normal. An increase of hemoglobin values, associated with persistently elevated reticulocyte levels and increased numbers of nucleated red cells in the marrow, occurred spontaneously in most patients despite little or no improvement in red cell survival. The rate of hemolysis was not influenced by cortisone. Except for initial large red cell volumes and except for loss of osmometric function together with increase in mechanical fragility, developing after 24 hours of sterile incubation, the patients' red cells were not measurably abnormal. Red cell "flattening" as seen in acute hepatitis and obstructive jaundice was not generally present. Serum iron and serum iron-binding capacity levels were both significantly reduced in the presence of normal or excessive deposits of hemosiderin in the liver and bone marrow.
Abstinence from alcohol was consistently followed by a rise in reticulocyte levels, occurring independently of diet and bed rest, suggesting that ingested alcohol suppresses the marrow response to the anemic state.

Susceptibility of Cells to Viral Infection. KARL M. JoHNson and Herbert R. Morgan,* Rochester, N. Y.

Viruses exhibit tissue tropisms and vary widely in their ability to grow in different tissues in vitro as well as in vivo, but factors responsible for this specificity remain largely undefined.

A model system, using psittacosis virus and chick embryo tissue, was devised to study this problem in vitro. Cultures of tissue lose their capacity to support virus multiplication when maintained in a nutritionally deficient culture medium of glucose and mineral salts. Virus production can be initiated subsequently in these cells by the addition of a chemically defined culture medium (No. 199 of Parker) containing numerous amino acids, vitamins, and accessory growth substances.

Furthermore, it has been shown that virus can enter these cells made deficient prior to its introduction in spite of the fact that multiplication fails to occur subsequently. Stimulation of virus growth occurs in the absence of cellular proliferation which can be prevented by the addition of colchicine.

Studies using various components of the chemically defined medium indicate that (1) fat-soluble vitamins, (2) certain purines and pyrimidines, and (3) ATP are not essential for reversal of the deficient state of the cells while (1) amino acids, (2) B vitamins, and (3) biological reducing agents are required for initiation of viral multiplication.

These studies of the nutritional requirements of psittacosis virus in deficient cells provide information concerning the factors which determine whether a tissue will support growth of a given virus, i.e., tissue tropism.

Studies on the Pulmonary Circulation: Effects of Anoxia, Exercise, and Hexamethonium in Patients with and without Thoracic Sympathetic Denervation. WALTER E. Judson, William Hollander and Julia G. Arrowwood, Boston, Mass. (Introduced by Robert W. Wilkins).

Of six patients who had had a transthoracic sympathectomy (D-1 to at least D-5) for arterial hypertension and tachycardia or for Raynaud's Disease, only three were effectively denervated as indicated by the presence of Horner's syndrome, by the absence of the peripheral arterial blood pressure "overshoot" to the Valsalva maneuver, and by a relatively fixed pulse rate during exercise. These three patients during the inhalation of 10 per cent oxygen for at least 15 minutes had no change in pulmonary arterial pressures, in cardiac output (Fick), or in pulmonary "capillary" pressure whereas the three other patients with incomplete denervations had rises in pulmonary arterial pressure without changes in cardiac output. In all cases the cardiovascular responses to exercise were normal. After intravenous hexamethonium the 
pulmonary arterial pressures usually fell without consistent changes in the calculated pulmonary arteriolar resistances.

A thoracic epidural or "differential" spinal block (procaine) was performed on six patients, two of whom were in congestive heart failure. On the basis of Horner's syndrome, pulse rate response to exercise, blood pressure response to the Valsalva maneuver, and sensory changes, only three patients had effective sympathetic denervation from at least D-1 to D-5. In these three patients, prior to the spinal block procedure there was a rise in pulmonary arterial pressure during the inhalation of 10 per cent oxygen (primarily as a result of an increase in the calculated pulmonary arteriolar resistance), whereas following the block there was no change in pulmonary arterial pressure, in cardiac output, or in pulmonary "capillary" pressure during anoxia.

Ten patients with compensated arterial hypertension, eight with mitral stenosis, and four with cor pulmonale were exercised and given inhalations of 10 per cent oxygen both before and after intravenous hexamethonium. After hexamethonium there was a fall in systemic arterial pressure, usually associated with a fall in pulmonary arterial pressure and pulmonary arteriolar resistance (without a change in cardiac output). However, when the cardiac output decreased after hexamethonium, the pulmonary arteriolar resistance increased, and it also increased when the lowered peripheral arterial pressure was restored to normal by intravenous norepinephrine. After hexamethonium an increase in pulmonary arterial pressure and arteriolar resistance still occurred during anoxia. Following hexamethonium patients developed less rise in pulmonary arterial pressure during exercise than they had before hexamethonium. Likewise they had a fall in calculated pulmonary arteriolar resistance during exercise instead of a rise as before hexamethonium.

It is concluded:

(1) Anoxia induces a rise in pulmonary arterial pressure through the activity of the thoracic sympathetic nervous system.

(2) Hexamethonium in the dosage used caused changes in pulmonary circulation apparently secondarily to the changes it produced in the systemic circulation and not through direct thoracic sympathetic paralysis.

Alterations in the Nature of the Adrenal Secretion Induced by the Prolonged Administration of ACTH to Rabbits. Edward H. Kass,* Oscar Hechter, I. Alden Macchi and Thomas W. Mov, Boston, Mass.

Differences between the effects of adrenocorticotropin and individual steroids of adrenal origin are becoming increasingly apparent. The adrenal glands of rabbits were reported to secrete predominantly corticosterone, yet this steroid did not significantly affect the structure and ribonucleic acid concentrations of rabbit lymph nodes, and depressed antibody production but slightly. ACTH, as well as hydrocortisone and cortisone, exerted marked effects on the lymph nodes and on antibody production. The paradoxical response to ACTH led to investigation of its effect on the adrenal steroid content of adrenal vein blood.

Corticosterone was found to be virtually the sole steroid component of adrenal vein blood of untreated control rabbits. There was no detectable hydrocortisone or cortisone. A single dose of ACTH did not significantly change the findings. However, when ACTH was given for one week, hydrocortisone was detected in a ratio to corticosterone of $1: 2$. When ACTH was given for 21-28 days, hydrocortisone became the predominant steroid, in a $4: 1$ ratio over corticosterone, and several unidentified trace components, not previously present, were demonstrable. The effects of chronic trauma are under investigation.

Thus, the qualitative nature of the adrenal secretion is not necessarily fixed genetically. There are probably two different actions of ACTH; the first is rapid and direct, and increases the steroid output of the adrenal gland without altering the nature of the secretion. The second effect is delayed, and may involve changes in the chemical nature of the secretory product, with different physiological and pathological effects.

Cardiac Output and Central Volume in Heart Disease as Determined by the Dye Dilution Technique. AldBRT Kattus, Arthur Rrven, Aaron Cohrn and Gilbert Sorto, Los Angeles, Calif. (Introduced by Samuel H. Basset).

The Hamilton dye dilution technique for the determination of cardiac output has been used to study the circulation in a variety of clinical states and in a group of normal subjects. From the slope of the down-stroke of the semi-logarithmically plotted concentration curves the central volume was calculated by the method of Newman. This central volume is believed to be closely related to the pulmonary blood volume.

Subjects were studied at rest in the post absorptive state. Cardiac output is expressed as the Cardiac Index (liters per square meter of body surface area) and central volume as Central Volume Index (cubic centimeter per square meter of body surface area). Average values for Cardiac Index (CI) and Central Volume Index (CVI) were determined in various categories.

In 7 normal subjects average CI was 3.5 liters per minute and CVI was 659 cc. In 23 pre-operative mitral stenosis patients CI was 2.4 and CVI was $481 \mathrm{cc}$. After commissurotomy, CI was 2.5 but CVI had increased to 543 cc. In 10 pre-operative aortic stenosis patients CI was 2.8 and CVI was 557. After valvulotomy CI increased to 3.5 but CVI did not change.

Patients with right ventricular failure (elevated venous pressure and edema) due to coronary arteriosclerosis and high output cor pulmonale had large CVI (723 to $1310 \mathrm{cc}$.). Those due to hypertension, aortic insufficiency, thyrotoxicosis and low output cor pulmonale had CVI less than the normal (392-582 cc.).

The studies indicate that congestive heart failure may be associated with a pulmonary blood volume less than normal. 
The Correlation of Human Gastric Potentials with Gastric Secretion as Measured by the Electrogastrogram. Irwin Katzka, Frederick Jackson, Jr. and Henry M. Lemon, Boston, Mass. (Introduced by Franz J. Ingelfinger).

The electrogastrogram has been studied by others to diagnose gastric disease but the physiologic significance of the intragastric potential so measured is not established. Investigations restricted to experimental animals have suggested that these potentials are produced by (1) the degree of acidity of gastric juice and (2) the electrical energy involved in the production of hydrochloric acid. We have carried out studies in man and have shown that these potentials are related to the process of secretion rather than change in concentration or acidity of gastric juice.

Using intragastric saline electrodes, calomel half cells and a new commercially available potentiometer, we recorded the potentials in the fasting stomach and following the administration of histamine or insulin in 45 normal subjects. With histamine, the potential increased rapidly from the fasting level and reached its peak before the maximum increase in gastric acidity occurred. With insulin, the rise in potential began 25-30 minutes later, correlating with hypoglycemia and again preceded maximum acidity. Gastric potentials were not increased by the introduction of acids, alkalies or gastric juice into the stomach. Furthermore, histamine produced no potential change from the stomachs of patients with pernicious anemia or from the esophagus or duodenum or skin of normals. In 3 out of 5 cases, intravenous Banthine decreased the potential independent of the changes in gastric pH.

The findings to date suggest that electrical potential is related to gastric function but it is independent of the character of gastric juice. The changes in electrical activity induced by the aforementioned drugs may be related to changes in the physicochemical activity of the gastric mucosa in the process of secretion and/or changes in the vascularity of the organ.

The Excretion of Acid Mucopolysaccharides in Normal Human Urine. GRACE P. KERBY,* Durham, N. C.

The amount of glucuronic acid excreted in the form of acid mucopolysaccharides in the urine of normal humans has been investigated as an approach to studying the metabolism of these substances. The fraction has been obtained from urine by the method described by Astrup.

The mean 24-hour excretion of acid mucopolysaccharides was $3.0 \mathrm{mg} \pm 0.6$ (S.D.) and $4.9 \mathrm{mg} \pm 1.3$ (S.D.) in white females and males, respectively. The difference between the two values was significant $(p=<0.01)$. Prostatic massage in one patient did not increase the amount of the material in urine. The material was present in full amount in urine obtained from the renal pelvis of one patient and thus was not derived to significant degree in this patient from structures lower in the urinary tract.
The urinary excretion of acid mucopolysaccharides by humans was markedly increased by the ingestion of glucuronolactone ( $5 \mathrm{gm}$ daily for several days).

Chromatographic studies of a further-purified fraction of acid mucopolysaccharides from normal human urine were done by a method developed during the present investigation. The major component of the fraction had the same $R_{2}$ value as chondroitin sulfate and produced a single spot when mixed with chondroitin sulfate, using a single solvent system.

The present information was sought to provide a background for the further study of certain metabolic disorders and hypersensitive states. Such studies are now in progress.

Alcohol, an Effective Inhibitor of the Release of Antidiuretic Hormone $(A D H)$. CharLES R. KIEEMan, Milton E. Rubini and EzRa Lamdin, New Haven, Conn. (Introduced by John P. Peters).

To evaluate the potential therapeutic effect of alcohol in conditions characterized by water retention, its action on the release of $\mathrm{ADH}$ in response to changes in volume, effective osmolarity and other variables has been studied. In contrast to numerous factors that enhance the activity of $\mathrm{ADH}$, only alcohol and water are known to inhibit it.

Semirecumbent normal men, under various conditions imbibed $120 \mathrm{cc}$. of bourbon whiskey. Electrolytes and freezing point depressions of serum and urine were measured. Urine flow (cc./min.) was divided into two fractions : Osmolar clearance $=\frac{\text { Urine osmolarity }}{\text { Plasma osmolarity }} \times$ Urine flow ; and Free water clearance = Urine flow - Osmolar clearance. Controls without alcohol were made in each series.

After alcohol free water clearance and urine flow rose, while excretion of sodium, potassium and chloride fell and a mild metabolic acidosis developed.

When a maximum urine flow was produced by sustained loads of water, alcohol had no effect on urine flow or free water clearance. It had a similar negative effect on a patient with diabetes insipidus.

The antidiuretic effect of hypertonic salt solution during maximal water diuresis was prevented by alcohol, which instead caused urine flow and free water clearance to rise further. Solute without water was, therefore, reabsorbed in a region of the tubules in which ADH presumably acts.

Prior alcohol imbibition minimized the antidiuretic effect of venous congestion of the lower extremities although excretion of solutes decreased.

The effect of alcohol was modified if it was given subsequent to the stimulation of $\mathrm{ADH}$ by hypertonic salt or venous congestion.

Alcohol did not improve the impaired excretion of water in Addison's disease.

Conclusions: Alcohol acts upon the supraoptico-hypophyseal system to block all stimuli provoking the release of ADH. This inhibitory action is being investigated in 
clinical states in which excessive activity of ADH has been hypothesized.

\section{Influence of Antibiotic Treatment on the Rate of Ac- quisition by Patients of Multiple Drug-Resistant Phage Group III Staphylococci Indigenous to a Hospital. Vernon KNIGHT, New York, N. Y. (Introduced by Walsh McDermott).}

At Bellevue Hospital, it was possible to determine the relative rates of acquisition of "hospital strains" of staphylococci by newly hospitalized patients receiving various therapeutic regimens. The determination was possible because a large majority of "hospital staphylococci" were of group III phage patterns resistant to penicillin, the tetracyclines, and streptomycin, in contrast to a negligible percentage of such strains in admission cultures. In the study, acquisition rates were estimated for $65 \mathrm{pa}$ tient carriers treated with penicillin, the tetracyclines, or no antibiotics.

Practically all tetracycline resistant strains were "hospital staphylococci," and they constituted about one-tenth of strains obtained at hospital admission. One-half of admission strains were penicillin resistant, the excess over one-tenth being strains not classifiable as "hospital staphylococci."

The rate of acquisition of "hospital staphylococci" was greatest among patients treated with tetracyclines, rising to 86 per cent in the 4-5-day period. It was second greatest among strains exposed to penicillin, not rising appreciably from admission values until the second week. The linkage between tetracycline and penicillin resistance among "hospital staphylococci" led to the paradoxical phenomenon that the rate of increase in penicillin resistance was greatest among strains from patients treated with tetracyclines. The rate of increase of "hospital staphylococci" was least among untreated patients.

Thus, there was demonstrated a close correspondence between the rate of acquisition of drug resistant "hospital strains" by staphylococcal carriers, and the degree of susceptibility of the strains they carried on admission to the agents used in treatment. It is postulated that the extent of elimination of susceptible strains by treatment determines the degree to which replacement with "hospital strains" may occur, and this hypothesis is supported by the observation that there is a low rate of acquisition of such strains by patients who are untreated.

\section{Studies on Antibodies Eluted from the Erythrocytes in Autoimmune Hemolytic Anemia. ZACHARIAS D.} Komninos and Muzaffer Arsoy, Boston, Mass. (Introduced by William Dameshek).

Antibodies eluted from the red cells in autoimmune hemolytic anemia were studied with respect to their thermal range of activity, ability to produce a specific type of Coombs' serum and reactivity with isolated fractions of red cell stroma. Twenty-nine eluates were studied from 11 patients with "idiopathic" and 4 with "symptomatic" hemolytic anemia. Dissociation of antibody from the red cells was achieved by exposure to low $\mathrm{pH}$, high salt concentration or high temperature.

Results: The titer of the eluates, even when cold hemagglutinins were present in the serum, was distinctly lower at $3^{\circ}$ than at $37^{\circ}$. Agglutination was also weaker in the cold and took longer to develop. Absorption experiments indicated that erythrocytes could remove more antibody at $37^{\circ}$ than at $3^{\circ}$. By injection of eluates into rabbits, Coombs' sera were produced ranging in titer from $1 / 4000$ to $1 / 10,000$. Coombs' sera of approximately the same titer were also obtained by injection of coated red cells from patients with autoimmune hemolytic anemia. These various sera were non-specific as they could agglutinate erythrocytes sensitized with iso $(R h)$-antibodies equally well. Normal human red cell stroma was extracted with $\mathrm{NaHCO}_{3}$ (fraction I) and with acetone (fraction II). Only fraction I could neutralize the antibody in all eluates tested. Boiling of this fraction destroyed its antibody neutralizing ability.

Conclusions: The antibody attached to the erythrocytes in autoimmune hemolytic anemia was of the warm variety in all cases tested. Coombs' sera obtained by in jection of eluates or coated red cells into rabbits were not specific for auto-antibodies. The red cell antigen with which the eluted antibodies reacted was extractable with $\mathrm{NaHCO}_{3}$ and heat labile, indicating its probable protein nature. The eluted antibodies of autoimmune hemolytic anemia have many of the characteristics of "immune" antibodies.

Influence of Certain Diseases on the Absorption of Vita$\min B_{12}$ from the Gastrointestinal Tract. JULIUS R. Krevans, C. Lockard Conley* and Marie Sachs, Baltimore, Md.

Vitamin $\mathrm{B}_{12}$ labelled with $\mathrm{Co}^{\infty}$ was administered orally to normal individuals and patients with various diseases. Stools collected for 6 days were dried, pulverized, and the gamma radiation measured under the same conditions used for the measurement of the radioactivity of the $B_{12}$ administered.

In 16 studies on normal individuals administration of 0.1-0.5 micrograms of labelled $B_{12}$ was followed by recovery of 10-47 per cent of the radioactivity in the stool. Within this range there was no relationship between the amount of $\mathrm{B}_{12}$ administered and the per cent recovered. With a dose of 1.0 microgram as much as 60 per cent of the radioactivity was recovered, and with 5.5 micrograms, more than 70 per cent.

In 23 studies on patients with diseases unrelated to pernicious anemia, administration of 0.1-0.5 micrograms of labelled $B_{12}$ was followed by recovery of 10-45 per cent. This group included individuals with achlorhydria, liver disease, and anemia unrelated to $B_{12}$ deficiency. One 21 year old male with hepatitis and normal gastric acidity in two studies excreted 67 and 68 per cent.

Eight patients with pernicious anemia excreted more than 60 per cent of the administered radioactivity of an administered dose of 0.5 micrograms. One patient who appeared to have typical pernicious anemia excreted less 
than 35 per cent in two tests. Further study by the technique of Schilling indicated apparently normal $B_{2} a b-$ sorption in this patient.

One of 2 patients with total gastrectomy and 2 of 3 with sprue were found to have impaired absorption.

One patient with megaloblastic anemia, sub-acute combined degeneration, normal gastric acidity, and multiple diverticula of the jejunum demonstrated markedly impaired absorption which became normal during the administration of aureomycin. Terramycin failed to produce normal absorption in a patient with pernicious anemia.

The Metabolism of Intravenously Infused $C^{\mathbf{1 4}}$ Labelled

Cholesterol in Man. George S. KurLand, JuditB $L$.

Lucas and A. Stone Freedberg,* Boston, Mass.

Preliminary observations of the metabolism of $\mathrm{C}^{\mathrm{At}}$ labelled cholesterol following intravenous infusion have been made in 3 euthyroid patients with angina pectoris. $C^{14}$ cholesterol, labelled in the 4 position in Ring $A$, was dissolved in saline with Tween 20 . Aliquots of plasma, red blood cells and stools were analyzed at frequent intervals for 13 to 110 days. The specific activity (S.A.) of cholesterol in various tissues was determined in one patient who expired 15 days after the infusion.

Following a period of rapid decrease in radioactivity, the disappearance of labelled plasma cholesterol was not a simple exponential function but consisted of multiple components. The S. A. of RBC cholesterol rose rapidly and reached equilibrium with plasma free cholesterol 7-14 hours after infusion. The S. A. of the plasma total cholesterol, reflecting the radioactivity of the plasma cholesterol esters, reached equilibrium with plasma free cholesterol 4-7 days after infusion. In the period immediately thereafter, the $\mathrm{T} 1 / 2$ of the RBC cholesterol, plasma free cholesterol, and total plasma cholesterol was 10.8 to 13 days. Subsequent disappearance rates were progressively slower; in one patient, for example, the S. A. of free cholesterol on day 110 was $84 \%$ that on the 80 th day after infusion. A "readily exchangeable cholesterol pool," determined by isotope dilution on day 7 and corrected for stool excretion, was 26 to 55 grams in the 3 patients. Daily fecal $\mathrm{C}^{16}$ radioactivity excretion was approximately $3 \%$ of the administered $C^{16}$ during the first 9 days after infusion; thereafter, a marked decrease in fecal radioactivity occurred. Fecal radioactivity was found in the sterol, fatty acid, and bile acid fractions.

At post mortem examination, in Case 2, the $S$. A. of liver cholesterol was the same as that of the plasma. Progressively lower specific activities were found in muscle, skin, fat, and atherosclerotic ressel, respectively.

The data are consistent with the hypothesis that the labelled carbon of the cholesterol molecule has been reutilized in the formation of new cholesterol.

The Diumal Variation in Adrenocortical Secretion. JoHN C. Laidlaw, Dalton Jenkins, Wiuliam J. Reddy and Thzodor Jakobson, Boston, Mass. (Introduced by George W. Thorn).

The diurnal variation in urinary 17 -hydroxycorticoid excretion has been studied on one or more days in 75 nor- mal subjects. The pattern is almost uniformly characterized by a higher level of excretion between 7 a.m. and 7 p.m. than during the overnight period. The fall in 17hydroxycorticoid excretion in the early evening does not usually persist until the following morning but is frequently succeeded by a rise in urinary levels around 3 a.m. Further confirmation of the predominance of the daytime excretion of 17-hydroxycorticoids was obtained from daily studies carried out over a 30-day period in three normal subjects; on only four occasions did the overnight excretion of 17-hydroxycorticoids exceed that of the day.

That the diurnal variation in 17-hydroxycorticoid excretion reflects predominantly an alteration in adrenal cortical secretion is suggested by experiments conducted on patients with Addison's disease given a maintenance dose of hydrocortisone (1-2 mg. per hour) by continuous intravenous infusion over a 24-hour period. In these patients, there was no predominance of day time excretion over that observed during the night.

Investigations of the mechanisms responsible for the diurnal variation in adrenal cortical secretion have indicated that the characteristic cycle persists despite enforced inactivity in bed, a reversal of the normal working schedule (night workers), a 24-hour period of starvation, a 24-hour period in which feedings were given every four hours and a reversal of the normal daytime predominance in urine output. The administration of ACTH during the night, however, leads to a reversal of the diurnal cycle. Further studies are being carried out in infants to determine the age at which the characteristic rhythm of adrenal cortical secretion becomes manifest.

These studies suggest that there is a basic diurnal regulation of adrenal cortical secretion, which is relatively resistant to changes in extrarenal environment.

The Metabolic Effects of Progesterone in Man. RICHARD L. Landau,* Delbert M. Bergenstal, Kathleen Lugibihl and Mary E. Kascht, Chicago, Ill.

It has been conclusively demonstrated that despite its restricted anabolic influence on the uterus and breast, progesterone, in approximately physiological amounts, promotes a general catabolic process. In three women and a man with apparently normal adrenal function $50 \mathrm{mg}$. per day of progesterone (intramuscular) induced a daily nitrogen loss which averaged 1-2 $\mathrm{gm}$. and persisted as long as the hormone was given (maximum 30 days). Uric acid excretion rose slightly, but no other indicators suggested an adrenal corticoid influence. Free urinary alpha amino acids and 17-keto-steroids were not increased, and circulating eosinophils rose. A moderate elevation in urinary sodium and a slight chloruresis were observed during the first four to six days of progesterone administration, and both elements were retained during the initial post-treatment phase.

In three patients with Addison's disease treated with cortisone and desoxycorticosterone the metabolic effects were much more intense, and the catabolic process continued for ten days after discontinuing the progesterone. 
The nitrogen loss averaged $2-4 \mathrm{gm}$. daily. Parallel losses of inorganic phosphorus and potassium were distinct and an enhanced creatinuria was observed in one patient. The increased urinary sodium and chloride excretion were also greater and more enduring, sodium losses again exceeding those of chloride. In a fourth Addisonian managed with salt alone progesterone did not affect sodium and chloride excretion indicating that the natriuresis and chloruresis were attributable to interference with the renal effects of salt retaining adrenal steroids.

The greater protein loss induced in patients with deficient adrenals suggests that this catabolic property of progesterone may be opposed by the adrenals, possibly by an anabolic secretion. The catabolic effects of progesterone and the interaction between it and adrenal steroids is important in the study of pregnancy, the menstrual cycle and the growth process itself.

Infrared Spectroscopy of Human Hemoglobins. DANIEL L. LARSON and JosepH McLaUghlin, Washington, D. C. (Introduced by George A. Perera).

Infrared spectra of normal adult hemoglobin, fetal hemoglobin, sickle hemoglobin and hemoglobin $C$ have been studied using a double beam recording infrared spectrophotometer. All samples were converted to carboxyhemoglobin before examination. Air dried hemoglobin films, cast from aqueous solution, showed almost complete loss of electrophoretic mobility. In order to avoid excessive infrared absorption due to the presence of water, hemoglobin samples were dried in vacuum from the frozen state and then dissolved in deuterium oxide or sodium deuteroxide. Hemoglobin solutions prepared in this manner showed essentially normal solubility and electrophoretic mobility.

Deuterated hemoglobin specimens were placed between silver chloride plates using platinum foil as a spacer and examined over a $\mathrm{pH}$ range up to 11.5. The various species of hemoglobin demonstrated differences in the rate of disappearance of the peak at $1545 \mathrm{~cm}^{-1}$ (6.47 microns) and the enlarging of the peak at $1440 \mathrm{~cm}^{-1}$ (6.93 microns). These rate changes appeared to be dependent upon the $\mathrm{pH}$ at which the examination was carried out. The significance of these variations in the infrared spectrum of the human hemoglobins will be discussed.

Urinary Excretion of Sodium and Potassium During the Pyrogenic Reaction. WilloughBy Latrex, New Haven, Conn. (Introduced by Paul B. Beeson).

Since serum and extracellular fluid electrolyte concentrations are usually maintained during fever, corrective renal adjustments apparently occur. Whether this compensation appears acutely when intrarenal vascular adjustments and hormonal mechanisms may be operative or only during prolonged pyrexia when extrarenal loss and internal shifts of water and electrolytes have occurred has not been determined.

The acute (three hours) effects of intravenous triple typhoid vaccine on urinary sodium and potassium ex- cretion, glomerular filtration rate (GFR-inulin clearance) and renal plasma flow (RPF-PAH clearance) were determined in normal subjects. After a latent period RPF and GFR usually decreased slightly and sodium, potassium and urine output fell sharply. Since electrolyte and water excretion diminished independently of changes in GFR, augmented tubular reabsorption occurred. After 30-45 minutes GFR returned to control levels, urine output rose, and RPF increased markedly. Sodium excretion rose abruptly and exceeded control values significantly in most subjects (on the average by $60 \%$ ). Potassium output continued to fall or returned toward control levels. Plasma sodium and potassium and venous hematocrit changed insignificantly.

Since similar results occurred in febrile and afebrile (aminopyrine premedication) subjects these acute changes are not attributable to fever or to extrarenal loss of electrolytes. The transient fall of both sodium and potassium excretion resembles the acute response to epinephrine/ norepinephrine and suggests that sympathetic discharge may have been responsible. However, hormonal mechanisms do not appear to account for augmented sodium output during hyperemia. The mechanism of this altered tubular activity is obscure, but such factors as undetected internal water and electrolyte shifts or possibly intrarenal hyperemia alone may have contributed. Since urinary sodium loss usually does not occur during prolonged pyrexia later adjustments apparently abolish this natriuresis.

The Transfer of Generalized Cutaneous Hypersensitivity of the Delayed Tuberculin Type in Man by Means of the Constituents of Disrupted Leucocytes. H. SHERwOod LAWrence,* New York, N. Y.

It is possible to induce, regularly, immunologically specific generalized cutaneous hypersensitivity of the delayed tuberculin type in animals and man by means of an injection of leucocytes obtained from sensitive donors.

In the studies outlined below, it has been found in the human, in contrast to animals, that leucocytes can be disrupted either by distilled water lysis or by repeated freezing and thawing and the resultant suspension of nucleoprotein sediment in cytoplasmic solution retains the capacity to transfer generalized delayed cutaneous hypersensitivity.

In 6 consecutive instances the constituents of distilled water lysed leucocytes and in 4 consecutive instances the constituents of repeatedly frozen and thawed leucocytes, obtained from the blood of donors sensitive to streptococcal $M$ substance, when injected into $M$-negative recipients resulted in the development of generalized delayed cutaneous $M$ substance hypersensitivity in each.

To substantiate this observation and to attempt identification of the particular cell component(s) involved in the mechanism of sensitization, the studies were repeated using tuberculin as the test material.

For 16 tuberculin negative recipients, suspensions of the constituents of repeatedly frozen and thawed leucocytes were prepared, on different occasions, from two tuber- 
culin sensitive donors. Each of 8 alternate recipients received an injection of such untreated suspensions; of the remainder, 4 recipients received desoxyribonuclease treated suspensions and 4 recipients received ribonuclease treated suspensions. In 16 consecutive instances generalized delayed cutaneous tuberculin hypersensitivity developed in each negative recipient and the pattern of response appeared similar in the test and control groups.

The data demonstrate that in the human the transfer of generalized delayed cutaneous hypersensitivity can be accomplished regularly with a suspension of the constituents of disrupted leucocytes and suggest that the mechanism responsible for this effect does not appear to depend upon the polymerized desoxyribonucleic or ribonucleic acid components of the donor cell.

\section{The Mechanism of the Osmotic Adjustment of the Body}

Cells. Alexander Leaf,* Jacques Y. Chatillon, Oliver Wrong and Elbert P. Tuttle, Jr., Boston, Mass.

There has been renewed interest in the adjustment of the body cells to changes in extracellular fluid concentration. A solution to this problem was obtained in vivo by determining the volume of distribution of a large intravenous water load from the dilution of the serum sodium and total solutes.

In fifteen initial experiments, a water load was administered rapidly to dogs whose excretion of water was minimized by Pitressin. The average volume of distribution of the water load was $65.6 \pm 3.2 \%$ and $63.6 \pm 2.3 \%$ of body weight as calculated from changes in serum sodium and total solute concentrations, respectively. This measures net movement of water through the body.

To compare the volume of distribution of a tracer molecule of water which requires no net movement of water, simultaneous measurements of total body water content by $\mathrm{D}_{2} \mathrm{O}$ were made in 10 additional experiments in dogs, either nephrectomized or with ureters ligated. The average total body water was $63.0 \pm 1.1 \%$ of body weight. The average volume of distribution of the water load was $61.2 \pm 1.5 \%$ and $67.6 \pm 1.8 \%$ of body weight as calculated from changes in serum sodium and total solute concentrations, respectively.

The close agreement between total body water and the volume of distribution of a large water load indicates that in vivo the osmotic adjustment of the overwhelming majority of body cells is accomplished by passive entry of water into the cells. Further conclusions from the data are: 1) The water load distributes itself so that the ratio of intracellular to extracellular water is unchanged. 2) Active transport of water to prevent changes in intracellular osmotic activity does not occur. 3) Osmotic equilibrium during dilution is not attained by osmotic inactivation of intracellular solutes or by their extrusion from the cells. 4) Significant release of sodium from bone or from within the cells in response to acute dilution does not occur.
$A$ Blood-born Vasoconstrictor Factor in Hypertensive and in Normotensive Subjects. RICHARD E. LEE, New York, N. Y. (Introduced by H. D. Lauson).

With the use of a new method of bio-assay, extremely low levels of vasoconstrictor activity can be detected in the body fluids of man and animals. The vasoconstrictor factor (VCF) present in such liquids as tissue washes, plasma, urine, and sera can be expressed in standardized physiological equivalents of epinephrine as gamma/cc.

The plasma level of VCF in hypertension and in normotensive patients and subjects is equal to $0.0001-0.001$ gamma/cc. epinephrine. It is relatively constant in each individual providing that the blood pressure is stable. Transient hypertension and severe hypotension are associated with highly significant increases in plasma VCF. In certain normotensive subjects, emotional trauma is accompanied by comparable changes in plasma VCF without blood pressure change, of note. Preliminary evidence is given associating high plasma levels of VCF with the "false positive" Regitine test.

The possible significance of this new factor is discussed in relation to human peripheral vascular physiology.

The Use of Tritium-and Radiocarbon-Labeled Precursors to Study the Metabolism of Steroid Hormones in Man. George V. LeRoy, Delbert Bergenstal, R. Gordon Gould, Harold Werbin and George T. Okita, Chicago, IIl. (Introduced by Clayton Loosli).

The role of acetate and diet cholesterol as precursors of adrenal corticosteroids was investigated by means of a double-isotope labeling technic, using three patients who underwent bilateral adrenalectomy and oophorectomy. For several days before surgery ACTH was injected; and tritiated cholesterol was fed daily in sufficient amounts to provide a nearly constant value for the specific activity of the plasma-free-tritium-cholesterol.

During the preoperative period, pooled urine samples were collected and the 17-ketosteroid fractions isolated were found to contain T-labeled ketosteroids. Approximately 11/2-2 hours before the adrenals were removed an intravenous dose of 1-C $\mathrm{C}^{14}$-acetate was given to "flash label" the cholesterol currently being synthesized by the patient. The urine collected during the first few days postoperatively contained, in addition to the $\mathrm{T}$-labeled steroids, $C^{14}$-labeled-17-ketosteroid fractions presumably formed during the two hours before removal of the adrenals and ovaries. In addition to the urine studies, determinations were made of the specific activities of tritium-and carbon 14-labeled adrenal cholesterol, and of the cholesterol in blood and other tissues obtained at surgery. The relationship between the specific activities of plasma-free-T-cholesterol, adrenal-free-T-cholesterol, and $\mathrm{T}$-17-ketosteroid indicated that diet cholesterol was a precursor of urinary ketosteroid. The specific activity of adrenal-free- $\mathrm{C}^{14}$-cholesterol, in every case, was several times greater than the specific activity of plasma-free- $\mathrm{C}^{\mathbf{1 4}}$ cholesterol, demonstrating that the ACTH-stimulated gland synthesized cholesterol rapidly from the $1-C^{14}$-acetate. Because of the fact that adrenal-free- $C^{16}$-cholesterol 
reached such high values so rapidly it is not possible to say whether the acetate went directly into steroid hormones, or whether the $\mathrm{C}^{14}$-ketosteroids were formed from the newly synthesized $\mathrm{C}^{14}$-cholesterol. The specific activity of the urinary. $\mathrm{C}^{14}-17$-ketosteroid was invariably less than that of the adrenal-free-C $C^{14}$-cholesterol. To accomplish these studies, methods were developed using a liquid scintillator for the determination of tritium and $\mathrm{C}^{14}$ in the same sample of plasma or tissue cholesterol, and in pure fractions of urinary steroid.

The Venous Pressure-Volume Curve of the Human Leg Measured in Vivo. Julius LitTer and JAMEs E. Wood, Boston, Mass. (Introduced by James M. Faulkner).

The purpose of this study was to develop a method for measuring venous pressure-volume curves of the leg which would be physiologically valid. Such curves should represent the effects of known venous pressures on venous volume.

A constant reference point or baseline value of venous volume at an effective venous pressure slightly greater than zero in all the veins was obtained when the horizontal leg was placed in a water-filled plethysmograph and the water pressure on the leg was greater than the natural local venous pressure. The actual venous pressure in the leg was adjusted to equal an arbitrary pressure in a venous congesting cuff on the thigh by "titrating" the water pressure on the leg and recording volume changes. Then the congesting cuff pressure was increased $30 \mathrm{~mm}$. $\mathrm{Hg}$ by increments of $5 \mathrm{~mm}$. $\mathrm{Hg}$ and the corresponding increases in leg volume were recorded. Experimental evidence will be presented indicating that these pressurevolume curves were produced by increasing the effective pressure in all the leg veins from slightly greater than zero to $30 \mathrm{~mm}$. $\mathrm{Hg}$ by increments of $5 \mathrm{~mm}$. $\mathrm{Hg}$.

The average total increase in venous volume was 4.75 cc. per $100 \mathrm{cc}$. of leg in healthy young men. Other studies have shown that the total vascular volume varies between $1.5 \mathrm{cc}$. and $1.0 \mathrm{cc}$. per hundred cc. of leg over a wide range of external pressures, the venous volume remaining constant at an effective venous pressure slightly greater than zero. Accordingly, the volume and distribution of blood in the human leg are mainly functions of the effective venous pressure.

The curves are not affected by hemodynamic changes and are determined by only one variable, the resistance of the veins to stretch. Therefore they are useful for the study of venous tone. This will be illustrated by the effects of epinephrine and norepinephrine.

The Response of Bone, Connective Tissue and Muscle to Sudden Changes in Extracellular Fluid Composition and Volume. M. F. Levitr, L. B. TURner, A. Y. SweEt and D. GrIBETz, New York, N. Y. (Introduced by Milton Mendlowitz).

Electrolyte and water analyses of cortical (marrowfree) bone, tendon, and muscle were performed in normal rats and in rats rendered acidotic, alkalotic, and salt-depleted during a four-hour interval. These states were produced by intraperitoneal administration of ammonium chloride, sodium bicarbonate and distilled water, respectively, in amounts calculated to augment total extracellular chloride or sodium or to deplete total extracellular salt by approximately $20 \%$.

Chloride acidosis induced: 1 . A reduction in extracellular phase of muscle of $15 \%$ as reflected in a reduced sodium and chloride space of muscle, together with a proportionately smaller increment in intracellular phase. 2. A fall of 0.025 meq., 0.015 meq. and 0.008 meq. of sodium/gm. of dry bone, tendon and muscle respectively, beyond that attributable to changes in extracellular fluid.

Sodium alkalosis induced: 1 . A $10 \%$ expansion of the extracellular phase of muscle as reflected in the increased sodium and chloride space of muscle, together with a reduction in intracellular phase.

Salt depletion induced: 1 . A predicted $20 \%$ reduction in the extracellular phase of muscle and a simultaneous expansion of the intracellular phase. 2. A fall of .020 meq. of sodium $/ \mathrm{gr}$. of dry bone and .015 meq. of chloride $/ \mathrm{gm}$. of dry tendon beyond that attributable to changes in extracellular fluid.

Approximately $90 \%$ of cortical bone sodium and a considerable fraction of connective tissue chloride and sodium do not appear to be contained in the extracellular fluid. Instead, such stores of salt are probably confined by the tissue matrix and may, in part, be rapidly released to maintain extracellular fluid composition and volume. Acute alterations in extracellular fluid $\mathrm{pH}$ appear to produce immediate changes in the volume of the extracellular phase of muscle, with reciprocal changes in intracellular volume.

The Relationship Between Changes in Thyroidal In Uptake and Serum Protein-Bound Iodine Following Thyrotropin. Richard P. LeVY, LUTHER W. KELLY, JR. and William McK. JefFeries, Cleveland, $O$. (Introduced by Max Miller).

A single intramuscular injection of thyrotropin (TSH) in normal persons produces a significant rise in the thyroidal uptake of $\mathrm{I}^{1 \mathrm{~m}}$ and in the serum protein-bound iodine (PBI) within 24 hours. The former reflects an increased rate of iodide trapping and the latter an increased release of thyroid hormone. A question arises regarding the relationship between these two effects of TSH stimulation, i.e. whether one might be dependent upon the other.

When thyroid extract is administered to normal persons the uptake of $\mathrm{I}^{212}$ is depressed, reflecting decreased endogenous TSH effect, but the response to injected TSH is still brisk. The PBI response, however, is not so consistent. After ingestion of comparatively large doses of thyroid over prolonged periods, no rise in PBI follows a single TSH injection. Presumably prolonged inhibition of endogenous TSH results in an atrophic thyroid gland which is not able to produce a significant rise in PBI in 24 hours, but which can still respond promptly with an increased trapping of iodide.

Studies of cases of hyperthyroidism following subtotal thyroidectomy or $I^{12}$ therapy have also produced evidence that these two effects are not necessarily interre- 
lated, even when the thyroid tissue is under strong endogenous TSH stimulation. One patient had a normal increase in $\mathrm{I}^{13}$ uptake in response to $\mathrm{TSH}$, but no rise in $\mathrm{PBI}$. In this instance the residual tissue was apparently forming and releasing thyroid hormone at a maximum rate, but the iodide uptake was not maximal. In two other patients TSH injection produced no rise in uptake, but a significant increase in PBI occurred. Here the uptake mechanism was apparently operating at a maximum rate, but that controlling the release of hormone was not.

Hence it appears that these two effects of TSH are not necessarily related, although under normal circumstances they usually occur together.

Studies of Inactivation and Formation of Antidiuretic Substance by Hypophysectomized Rats. CharLES W. Lloyd,* Erich H. Loewy and Sophie Pierog, Syracuse, N. Y.

Normal and hypophysectomized young adult male Sprague-Dawley rats were pair fed a diet high in calories and protein which maintained good growth rates in both groups. The hypophysectomized animals had greater fluid intake and urine volume than the normal animals. Daily administration of Pitressin Tannate in oil (1 milliunit/ gram) caused a greater decrease in fluid intake and output in the hypophysectomized rats and this was maintained throughout the administration of Pitressin. The normal animals had a decrease in urine volume lasting for the first few days of Pitressin Tannate administration but had no change in fluid intake. Hyponatremia and hypochloremia developed in the normal but not in the hypophysectomized animals. This difference was probably due to greater dilution in the normals. The livers of the pairfed hypophysectomized animals had normal ability to inactivate Pitressin in vitro. Daily priming with Pitressin did not alter this ability in either group. Since hypophysectomized animals which became cachectic on a diet of ordinary laboratory pellets had a marked decrease in hepatic ability to inactivate Pitressin, it appears that this defect is primarily the result of nutritional rather than endocrine factors. Serum of recently operated animals had no activity. Serum of many animals hypophysectomized for $\mathbf{3 0}$ days contained essentially the same amount of antidiuretic activity as was found in normal serum. This suggested that reorganization had taken place, permitting release of $\mathrm{ADH}$ from the hypothalamus. Measurement of hypothalamic antidiuretic activity demonstrated little activity immediately after operation. The hypothalamic antidiuretic activity of animals operated one month was higher than in normal animals. This activity was concentrated in the area containing the median eminence. It was decreased by dehydration and increased by hydration. Pentobarbital appeared to increase the antidiuretic activity of the hypothalamus.

Melanuria. George D. Ludwig, Philadelphia, Pa. (Introduced by Francis C. Wood).

Melanuria is usually associated with metastatic melanoma. However, we have observed it in a variety of diseases, and suggest possible alterations of pigment metabo- lism that may have caused it. Melanin, or its precursor, was demonstrated spectrophotometrically, by five specific chemical tests, and by isolation of the pigment, in the following conditions: chronic liver disease with hyperpigmentation, peri-cholecystic abscess, hemochromatosis, argyria, Addison's disease, and metastatic melanoma.

Melanin is a polymer derived from tyrosine by a series of reactions involving the enzyme, tyrosinase, and autooxidative steps. In man, the metabolic fate of tyrosine includes urinary excretion, enzymatic degradation in the liver, and conversion of small amounts to epinephrine, thyroxine, and melanin.

In chronic liver disease, the normal mechanism for metabolizing tyrosine may be depressed as evidenced by tyrosinemia and tyrosinuria. Increased supply of substrate to tyrosinase may be an important factor leading to hyperpigmentation and melanuria occasionally seen in these patients, although other factors are involved.

Local tissue necrosis occurred with the peri-cholecystic abscess. Pigment production may have resulted from increasing substrate by liberation of phenolic groups of proteins, by $\mathrm{pH}$ changes, or from decrease of sulfhydryl groups, which have been proposed as normal inhibitors of tyrosinase.

Increased dermal melanin deposition commonly found in hemochromatosis and argyria, and the melanuria may result from sulfhydryl binding or from direct acceleration of the enzymatic reaction by metal ions. Both phenomena have been demonstrated in vitro.

Melanuria was found in chronic Addison's disease with hyper-pigmentation, but not in acute surgically induced hypoadrenalism. The mechanism of increased pigment production in Addison's disease is unknown, but the recent proposal that the adrenal regulates tissue sulfhydryl groups is in best agreement with present experimental data.

Spectral Phonocardiography: Studies of Heart Sounds and Murmurs with a Modified Bell Sound Spectrograph. Victor A. McKusick, Samuel A. TAlbot and Grorge N. WebB, Baltimore, Md. (Introduced by $\mathrm{E}$. Cowles Andrus).

The Bell sound spectrograph was adapted for phonocardiography by these modifications: 1) Analyzing passband filter widths of 25-35 c.p.s. provide optimum simultaneous resolution in time and frequency. 2) Parallel recording of EKG, etc., has been instrumented. 3) A logarithmic display of frequency spectrum has a more realistic appearance and advantageously "spread out" the lower frequency range where greater energy content is concentrated.

Spectral phonocardiograms incorporate all three parameters of heart sounds and murmurs in a single, detailed, analytical display.

Time is displayed on the abscissa, frequency spectrum on the ordinate; intensity range is represented by degree of blackness of particular portions of the records (on electrically sensitive paper). Precise frequency $X$ intensity analyses at specific points in time are also possible. 
Spectral phonocardiograms resemble the mental images of cardiovascular sounds. Their interpretation is easy for the experienced stethoscopist. Their value in teaching and for permanent clinical records is great. Artefacts due to ambient vibrations or to electrical interference can be recognized as such and disregarded.

Quantitative analyses of frequency content are presented. Some murmurs and altered heart sounds have frequency components at 1500 c.p.s. or even higher. Higher frequency components are important in stethoscopy because of the Fletcher-Munson characteristics of the ear.

The phonetics of the heart, i.e., the physical basis of many adjectives used in describing the quality of these sounds, is elucidated: e.g., "cooing" diastolic murmurs are characterized by a clear-cut fundamental with harmonics and a distinctive curvature. Fundamental-harmonic systems have also been found in musical systolic murmurs. (The physical basis for the terms applied to percussion notes, breath sounds, voice sounds, and rales has been investigated.)

We seek information which is otherwise unobtainable clinically and which may be useful in the differentiation of sounds of islentical stethoscopic character but different etiologic, prognostic, and functional significance.

Clinical Application of a Sensitive Method for Detecting Antihormones in Patients Receiving Pituitary FSH. William O. Maddock, Ichiro Tokuyama, Robert B. Leach and C. Alvin Paulsen, Detroit, Mich. (Introduced by Gordon B. Myers).

Conventional methods for detecting antihormone formation in patients receiving pituitary FSH are based upon the ability of the patients' plasma to prevent the effects of the FSH administered to immature female rats or mice. These methods are so insensitive that clinical evidence of refractoriness usually appears before antihormone activity can be demonstrated in the plasma, and antihormones usually are no longer detectable one to three months after stopping treatment. The antihormones which develop in patients receiving pituitary FSH preparations are capable of blocking the action of rat pituitary FSH. This suggested a sensitive antihormone assay method, based on the fact that the presumably minute amounts of endogenous FSH circulating in the immature female rat are necessary for chorionic gonadotrophin (CG) to stimulate ovarian estrogen secretion. Thus, to prevent the action of CG (increased estrogen secretion as evidenced by enlarged uteri) only enough antihormone need be present to block the assay rat's endogenous circulating gonadotrophin. This method was compared with the conventional assay for detecting antihormones in six patients who received hog pituitary FSH for 2 months or longer. The "CG method" was 10 to 20 times more sensitive than the conventional assay and permitted detection of antihormones at the onset of clinical refractoriness and for as long as $\mathbf{1 2}$ months after stopping FSH treatment.

In keeping with the interpretation that the antihormones block CG action indirectly, by neutralizing the female rats' endogenous gonadotrophin, the antihormones did not block the direct, Leydig cell-stimulating action of CG in male rats which does not require the presence of pituitary gonadotrophin. In two men having high antihormone levels as the result of hog FSH therapy, CG therapy produced a prompt response as judged by increased urinary estrogens and 17-ketosteroids and symptoms of enhanced androgen production.

Succinic Dehydrogenase Activity in Tuberculous Animals. S. P. Martin,* S. N. Chaudhuri and C. D. Cooper, Durham, N. C.

In studies on the metabolic response of guinea pigs to tuberculosis it was noted that the non-caseating tissues, such as kidney, had a low succinic dehydrogenase activity. This loss of activity was also noted in animals suffering from hemorrhagic shock. The activity could be restored by addition of a factor present in normal kidney cortex.

Chromatographic studies of normal tissues on paper and ion exchange column revealed that a nucleotide was responsible for activation. This nucleotide contains adenine, ribose, two phosphate groups and three alpha amino groups. There is a free sulfhydryl after treatment with cyanide and Toennes reagent. The factor is thermolabile. Unchromatographed adenine diphosphate contains an impurity that will serve as a basis for the nucleotide when tissues are incubated with glutathione, cysteine and cystine. Dinitrophenol blocks the restorative capacity of the nucleotide. This effect cannot be produced by the known nucleotides including coenzyme A.

These data would indicate that animals in shock or severely infected with tuberculosis have a low succinic dehydrogenase activity. This lowered activity appears to be related to a lowering of a tissue nucleotide. The nucleotide may operate in one of three ways: It may remove oxalacetate from the system. It may be related to the Slater factor. Also, this compound might serve as as a link in oxidative phosphorylation. The latter speculation has the greatest experimental proof. The loss of this material by the cell may play a role in the pathogenesis of this disease.

\section{Laboratory and Clinical Studies of a Modified Hemolytic} Test for Tuberculosis. C. MIDDlebrook,* H. Ozerengin and R. Oestreicher, Denver, Colo.

The unsatisfactory results obtained by many investigators on application of the originally described hemolytic test for the diagnosis of tuberculosis led us to investigate the causes of false positive reactions in this test. The following causes have been discovered and eliminated:

1. A serologic cross-reaction exists between a component of old tuberculin and Staphylococci: a special method of fractionation of tuberculin has permitted removal of this component from the specific red-cell-sensitizing antigen.

2. A labile red-cell-lyzing substance which appears in some sera from non-tuberculous individuals can be inactivated by treating the diluted serum with calcium ions 
at $37^{\circ} \mathrm{C}$. for two hours before submitting it to the hemolytic test.

3. A serologic cross-reaction between tuberculin and histoplasmin, causing the sera of certain patients suffering from histoplasmosis to give a false positive reaction in the hemolytic test for tuberculosis, can be eliminated by preliminary absorption of such sera with small amounts of histoplasmin - a procedure which has no effect on the titres of sera from tuberculous individuals.

Application of the hemolytic test, modified as a result of identification of the above causes of false positive reactions, in the study of over 1,300 sera from non-tuberculous groups of human beings gave less than $3 \%$ false positive reactions. The same modified test was applied to sera from a large number of tuberculous individuals. Approximately $75 \%$ of untreated cases gave "doubtful" or "positive reactions." It has also been observed that chemotherapy with isoniazid and streptomycin has a "provocative" effect on humoral antibodies as measured by the hemolytic test: more than half of the untreated cases which gave "false negative" reactions before therapy became serologically positive in the hemolytic test within a few months after initiation of such therapy.

It is concluded that the modified hemolytic test has proven useful as a diagnostic test for tuberculosis.

Total Liver Phosphorus: Its Relation to Hepatic Glycogen Deposition. Nancy Nichols, Fredy E. Schertenletb and EMery C. Mirler, Boston, Mass. (Introduced by Alexander Marble).

Costisone, administered for 18 days, caused a $100 \%$ increase in liver weight in both normal and alloxandiabetic rabbits. The increase in liver size was due entirely to the intracellular deposition of glycogen and water. The percentage of intracellular water in the whole liver was unchanged and its potassium concentration remained constant.

In contrast to the increase in concentration of acidsoluble phosphate associated with hepatic glycogenesis, the concentration of total phosphate fell from $245 \mathrm{mM} / \mathrm{Kg}$ intracellular water in glycogen-free livers to 126 in livers in which glycogen constituted $50 \%$ of the dry solids. A constant P:N ratio of 3.73 (mM phosphate: Gms nitrogen) was found in all livers. In the intracellular water of glycogen-free liver, total phosphate was so high that more than half must have been bound, possibly to protein, if the criteria of osmotic equality and electroneutrality are to be satisfied. It is postulated that this excess phosphate, present in liver but not in muscle, forms an intrahepatic reservoir of phosphate ions which, as glycogen is deposited, can readily change their state, entering the pool of acid-soluble phosphate and supplying anion equivalence and osmolar force to the potassium and water which are deposited with glycogen.

Furthermore, since it appears from these data that no extrahepatic phosphate enters the liver during glycogenesis, it is concluded that the fall in serum phosphate associated with the intravenous administration of glucose is related exclusively to the peripheral utilization of carbohydrate.
The Role of Venomotor Activity in Circulatory Adjustments. Ernest B. Page, Herbert O. Sieker, John B. Hickam * and William W. Pryor, Durham, N. C.

Venomotor activity is involved in normal and abnormal circulatory dynamics, but its magnitude and importance have been difficult to assess. The present study is concerned with methods of measuring venous reactions, the pattern of normal venomotor responses, and venomotor abnormalities in cardiovascular disease.

Changes in central venous pressure, reflecting venomotor activity in the body as a whole, were compared with pressure changes in a superficial vein segment isolated by external pressure (Duggan). In sixteen normal subjects observations have been made on responses to the Valsalva maneuver, cold, exercise, postural change, and hyperventilation. After the Valsalva maneuver, which produced the greatest and most consistent response, there was a mean rise of $16 \mathrm{~mm} \mathrm{Hg}$ in the vein segment and 4 $\mathrm{cm} \mathrm{H}_{2} \mathrm{O}$ in central venous pressure. Tilting and hyperventilation also caused pressure rises in both regions, though less marked. With cold and exercise the superficial segment constricted well, but central venous pressure changes were inconsistent. Since the two regions do not always change together, it appears that different stimuli can invoke different patterns of venous constriction. This venomotor activity has obvious importance in controlling the distribution of blood volume throughout the body.

Four patients with diabetic, idiopathic, and post-sympathectomy hypotension showed markedly impaired or absent venous responses to the standard stimuli. Stimulation of a sensitive carotid sinus, or coughing, in a patient with cough syncope produced simultaneous falls in central venous and arterial pressures without change in heart rate. It is concluded that impairment of venomotor activity, with consequent decrease in venous return to the heart, plays a significant part in the disability of such patients.

Pituitary Somatotropin as a Growth for Human Mammary Carcinoma. Olof H. Pearson,* Bronson Ray, Charles D. West, Charles C. Harrold, John P. Maclean and Min Chiu LI, New York, N. Y.

Oophorectomy and adrenalectomy will induce objective remissions in patients with estrogen dependent mammary cancer. Olivecrona and Luft have reported improvement from hypophysectomy in patients with metastatic mammary carcinoma. The question arises whether hypophysectomy induces improvement simply by suppression of gonadal and adrenal function, or whether there is in addition a pituitary hormone which is important in maintaining the growth of breast cancer.

Two women with active metastatic osteolytic mammary carcinoma, who had previously undergone oophorectomy and adrenalectomy with transient improvement, have undergone hypophysectomy. Both patients have obtained objective remissions as evidenced by improvement of severe myelophthisic anemia, cessation of osteolysis, and calcification of osteolytic lesions in one patient. The remissions continue 7 and 3 months after hypophysectomy. 
Beef pituitary somatotropin was administered intramuscularly in doses of 100 milligrams daily for 18 days to one of these patients three months after hypophysectomy, while on a metabolic balance regime. During growth hormone administration, there was a steady rise in urinary calcium excretion from 165 milligrams to 408 milligrams per day. There was a concomitant rise in urinary phosphorus and in fecal calcium and phosphorus excretion. Nitrogen and potassium balances became slightly negative during growth hormone administration. When somatotropin was stopped, urinary calcium and phosphorus excretion promptly returned to control levels. These data indicate that growth hormone induced osteolysis presumably due to stimulation of growth of osteolytic tumor.

These observations suggest that pituitary somatotropin is an important growth factor for human mammary carcinoma.

Studies on a Salt Retaining Factor Prepared from Human Urine. Maurice M. Pechet, Leroy E. Duncan, Jr., Grant W. Liddle and Frederic C. Bartter,* Bethesda, Md.

A salt retaining factor was prepared from human urine by the following method. The urine was acidified to $\mathrm{pH}$ 1 with hydrochloric acid, extracted exhaustively with ether and methylene chloride, and the methylene chloride extract washed with alkali and water. The carefully dried extract was subjected to silica gel column chromatography. Fifteen fractions were eluted. Bioassays for sodium retention and potassium excretion in adrenalectomized dogs indicated that the active material was eluted with methylene chloride-methanol mixture. This fraction was subjected to paper partition chromatography in the Pechet system. The migration rate of the active material was different from those of cortisone and hydrocortisone. The material was characterized by ultraviolet absorption, reaction with alkaline silver nitrate and blue tetrazolium. Of the various urines studied the material obtained from urine of patients with cirrhosis and ascites showed the highest degree of increased activity over that from normal subjects.

Evaluation of the salt retaining activity of this factor when administered to human subjects will be discussed.

\section{Abnormalities of Carbohydrate Metabolism of Red Cells in Hereditary Spherocytosis. Thomas A. J. Prankerd, Kurt I. Altman and Lawrence E. Young, * Rochester, N. Y.}

Since many hereditary diseases are known to be due to defects in intermediary metabolism, experiments were designed to determine if such an abnormality could be detected in red cells from patients with hereditary spherocytosis (HS).

Normal and pathological human erythrocytes were incubated with $\mathrm{NaH}_{2} \mathrm{P}^{22} \mathrm{O}_{4}$ at $37^{\circ} \mathrm{C}$ with constant bubbling of $95 \% \mathrm{O}_{2}$ and $5 \% \mathrm{CO}_{2}$ to insure mixing and constant $\mathrm{pH}$. Phosphate intermediates involved in glycolysis were separated unequivocally from trichloracetic acid extracts of "intracellular" and stromal fractions of these cells by paper chromatography.

Normal erythrocytes take up $\mathbf{P}^{2}$-phosphate from extracellular medium at reproducible rates, and the distribution of radioactivity within the cells follows a constant pattern. 2,3-diphosphoglyceric acid (DPG) bears a precursor relationship to adenosine triphosphate (ATP) and both fractions show greater activity than inorganic phosphate. Stromal fractions show moderate activity of both 2,3-DPG and ATP with magnitudes and time courses different from those demonstrated intracellularly. Incubation with adenosine has only slight effect on relative specific activities (RSA's) during the first six hours.

Cells from HS patients, before and after splenectomy, take up $\mathrm{P}^{x 2}$-phosphate at the same rate as normal cells, but relationships among intracellular fractions differ markedly, the RSA of inorganic phosphate exceeding that of either 2,3-DPG or ATP. Stromal fractions have low activities. Cells from certain of these cases incubated with adenosine show intracellular relationships restored closely to normal, but stromal activities are not affected. Phosphate metabolism in cells from other HS cases is not altered significantly by adenosine. This and other biochemical findings, together with recent hematological observations, indicate that the group of cases in this clinic regarded as examples of HS may not be homogeneous.

Results such as these suggest that "inborn errors" of erythrocyte metabolism may be partly responsible for the hematological features of hereditary spherocytosis and other congenital hemolytic disorders.

\section{$A$ Study of the Use of $R b^{*}$ as a Tracer for Measurement of $R b^{\infty}$ and $K^{\infty}$ Space and Mass in Intact Man with and without Congestive Heart Failure. C. THORPE RAY,* Sam Threefoot and G. E. Burch,* New Orleans, La.}

Since rubidium enters potassium metabolic pool, attempt was made to estimate $\mathrm{Rb}^{*}$ space and potassium space and mass, utilizing radiorubidium. $\mathbf{R b}^{\mathbf{\omega}}$ (half-life 19.5 days) provided information about potassium metabolism for prolonged periods (70 days) unobtainable with $K^{4}$. One control and two subjects with heart failure were studied under controlled metabolic conditions. Intracellular (erythrocytes) and extracellular (plasma) compartments were studied simultaneously.

Estimation of space and mass by isotope dilution assumes equilibrium of distribution of isotope in all compartments. Absolute equilibrium of distribution was not achieved since (1) erythrocyte $\mathrm{Rb}^{*}$ increased 5-8 days after injection, whereas plasma $R^{*}$ decreased, (2) plasma $\mathrm{Rb}^{*}$ declined independently of erythrocytes and (3) both plasma and erythrocyte $\mathbf{R b}^{*}$ declined more rapidly than could be explained by excretion from body. This would likewise apply to $\mathrm{K}^{\mathrm{a}}$.

Although estimated potassium space and mass utilizing erythrocyte space and mass equivalents varied less than estimations based on plasma determinations, enough temporal variation existed to indicate unreliability of calculations based on single observations. Space and mass 
calculated from plasma values increased progressively, reflecting more rapid excretion from plasma than from cellular compartments. Increased potassium intake caused more rapid $\mathrm{Rb}^{*}$ loss from plasma than erythrocytes, marked increases of space and mass utilizing plasma space and mass equivalents, but only slight increases using erythrocyte space and mass equivalents. Therefore, in estimating space and mass of substances, the major compartment should always be sampled, i.e., for primarily intracellular substances $(\mathrm{Rb}, \mathrm{K})$, the intracellular compartment, and for primarily extracellular substances $(\mathrm{Na}, \mathrm{Cl})$, the extracellular compartment.

Utilizing average erythrocyte values $R^{* 6}$ space was 54.6, 49.1 and $43.5 \%$ body weight and potassium mass was $136.7,104.3$ and 91.0 grams or $0.196,0.186$ and $0.164 \%$ body weight for control, patient with moderate CHF and severe $\mathrm{CHF}$ respectively. These values increased slightly on higher potassium intake.

The Relation of Urinary Ammonia to Urine $p H$. FLoYd C. Rector, Jr., Albert D. Roberts, Jr. and JoHN Copenhaver, Dallas, Tex. (Introduced by Ben Friedman).

The rate of hydrogen ion secretion has been considered to be the factor regulating ammonia excretion. To elucidate this relationship, $\mathrm{H}^{+}$formation was selectively blocked by giving rats carbonic anhydrase inhibitor (Diamox) for 14 days. Urine was analyzed for $\mathrm{pH}$, titratable acid, and ammonia. Serum and muscle electrolyte composition was determined. Activities of glutaminase and carbonic anhydrase were measured in kidney homogenates.

Diamox resulted in a sustained rise in urine $\mathrm{pH}$ and fall in titratable acid excretion. Despite the alkaline urine, ammonia excretion rose from $1.71 \pm .20$ meq. daily in controls to $2.27 \pm .25$ meq. daily with Diamox alone and $2.32 \pm .28$ meq. daily with Diamox $+\mathrm{KCl}$. Paralleling the increased ammonia excretion renal glutaminase activity rose from $788 \pm 39$ units $(\mathrm{M} \mathrm{NH} / 100 \mathrm{mgm}$. dry kidney/hour) in controls to $2246 \pm 487$ units with Diamox alone and $1910 \pm 193$ units with Diamox $+\mathrm{KCl}$. Intracellular $\mathrm{K}^{+}$decreased from $51.4 \pm 1.2 \mathrm{meq} .100 \mathrm{gms}$. fat-free dry muscle (FFDM) in controls to $37.9 \pm 6.1$ meq./100 gm. FFDM in experimental animals.

Potassium deficiency cannot be responsible for accelerated ammonia excretion in rats given Diamox, since correction of the potassium deficit prevented neither increased ammonia excretion nor increased glutaminase activity. The possibility of intermittent Diamox inhibition, with consequent post-inhibitory rebound phenomena, was eliminated by giving the inhibitor at $4-8$ hour intervals. Following a single injection of Diamox there was still approximately $25 \%$ inhibition of carbonic anhydrase activity at the end of 8 hours. Therefore, significant inhibition was maintained at all times.

Increased urinary ammonia in an alkaline urine indicated that ammonia excretion was at least partially independent of urine pH. It is suggested that the increased ammonia excretion was the consequence of increased renal glutaminase activity, which may have been related to changes in intratubular acid-base equilibrium.

Mitral Stenosis: The Effects of Digoxin with Exercise. E. A. S. Reid, A. B. Judd, D. J. Macintosh, I. G. Milne, T. R. Hale and M. McLaughlin, Montreal, Canada. (Introduced by J. S. L. Browne).

Although investigators have reported upon the effects of Digoxin on the function of the human heart modified by mitral stenosis while the patient was at rest, the effect of this drug during exercise appears to have been neglected. The present paper is a preliminary communication on this apparently unexplored problem.

Eleven patients with mitral stenosis were studied. Cardiac catheterization was performed and pressures and outputs determined. Exercise by leg flexion was carried out for five minutes and the data recorded. One milligram of Digoxin was administered intravenously. Studies were repeated after one hour.

Four patients, compensated and with normal rhythm, showed minor changes only, following digitalization.

Three patients, decompensated and with auricular fibrillation, showed improvement at rest in rate, output and diastolic pressures. With exercise there was little improvement over the control period. Diastolic pressures rose to a less degree.

Three patients, decompensated but with normal rhythm, showed the most improvement. The outputs were increased at rest and with exercise following Digoxin. At rest diastolic pressures fell but systolic pressures were unchanged. With exercise, there was a marked rise in systolic pressure during the control period but following Digoxin there was no rise at all.

These studies suggest that Digoxin has an effect, not only on the failing ventricle but also on the failing auricle when this auricle has a normal rhythm. We have shown that Digoxin has markedly improved the function of exercising "mitral stenotics" in failure when sinus rhythm is present, and that this improvement is absent when the auricle is fibrillating. In decompensated mitral stenosis after exercise, we conclude that Digoxin has a greater beneficial effect when normal rhythm is present than when the auricles are fibrillating.

Factors Influencing the Growth of Megaloblasts In Vitro: Effect of Vitamin B-12, Folinic Acid (CVF), AMethopterin and Colchicine. EDWARD H. REISNER, JR. and Harold T. Swan, New York, N. Y. (Introduced by L. Emmett Holt, Jr.).

Others have reported that megaloblasts grown in suspension cultures in PA serum showed normoblastic "ripening" with the addition of folic acid, but not with B-12 unless the latter was accompanied by intrinsic factor contained in normal gastric juice. We found that using their technique (a modified Osgood culture) when grown in suspension in PA serum for 48 hours megaloblastic marrows from PA patients showed a mean increase of nucleated red cells per $\mathrm{mm}^{3}$ of $308 ; 561$ with added B-12; 282 with intrinsic factor; 490 with B-12 plus intrinsic 
factor, and 1028 with CVF. Only the latter change was statistically significant $(p<.05)$. Maturation of cultures was accompanied by disappearance of megaloblasts and increase of normoblasts, more marked in the presence of B-12 or CVF. In contrast, when grown on chick plasma clots or glass surfaces B-12 alone appeared to be strongly stimulatory and to restore normoblastic hematopoiesis. (CVF was not tested by the latter methods.)

The increase of cells in megaloblastic marrow suspension cultures could be blocked with colchicine indicating it was due to mitosis. Normoblastic marrows showed no greater increase of nucleated red cells after 48 hours when CVF was added than in the control. AMethopterin caused a decrease in cells which was completely reversed by CVF but not by folic acid.

Failure of suspension marrow cultures to be stimulated markedly by B-12 in contrast to cultures on clots and glass surfaces is surprising. Possibly, the minute quantities of B-12 present in relapsed PA serum are inadequate to support the more vigorous growth of the latter cultures but suffice for the limited mitotic activity in suspension. Since both B-12 and CVF must be present in minimal amount for mitosis, under such circumstances the amount of CVF present in the suspension could become the major factor influencing growth.

These studies fail to support the hypothesis that B-12 must be "activated" by intrinsic factor to function in hematopoiesis.

Studies in Endothelial Capillary Permeability: II. Congestive Failure. Alexander P. ReMenchIK and RoBERT M. KARK,* Chicago, Ill.

Reliable methods for simultaneous determinations of red cell mass (space) and human serum albumin space were developed. These were applied to measurement of the spaces in patients with heart disease, before and after successful therapy of congestive failure. Red cell mass measures intravascular space. In congestive failure it is "normal" and does not change following successful therapy. Albumin space measures intravascular space plus an unknown portion of the extravascular compartment. The albumin space is increased in congestive failure and this implies that endothelial permeability to albumin is also increased, since albumin space shrinks to "normal" after therapy. Using data from others, together with our own observations, an hypothesis for the pathogenesis of congestive failure is presented and the physical findings in this condition are explained. In patients with heart disease, the decrease in myocardial efficiency produces a change in permeability of the capillary endothelium; the development of this change needs study. Transfer of protein to the extravascular compartment causes a fall in the quantity of intravascular protein and a transient decrease in intravascular volume. Homeostatic mechanisms for maintenance of blood volume are activated, among which is retention of sodium and water. These, with protein, enter the extravascular compartment. In addition, there occurs a reflex spasm of the venous "off-flow system" which leads to increased periph- eral resistance and distention of the large veins. The above mechanisms account for the cold, cyanotic extremities, the distended liver, the distended veins, and the raised venous pressure seen in congestive failure. Peripheral space may be relaxed by injection of TEAC which produces a fall in venous pressure and loss of venous distention in patients with congestive failure. Biochemical Sequence of Events After Insulin Adminis-
tration to Diabetic Rats. ALBERT E. RENOLD and A. Baird Hastings, Boston, Mass. (Introduced by Kendall Emerson, Jr.).

Whereas effects of insulin added in vitro to skeletal muscle preparations can be demonstrated with ease, effects of insulin added in vitro to hepatic tissue are most difficult to obtain. Indeed, the only clear-cut effect of insulin added in vitro to isolated liver tissue concerns the incorporation of acetate into fat, while attempts to demonstrate an insulin action on glycogen deposition, glucose utilization or glucose oxidation to $\mathrm{CO}_{2}$, have been negative. This is particularly puzzling since marked differences between normal and diabetic liver incubated in vitro have been shown. In this study an attempt has been made to define the biochemical sequence of events during the reversion of diabetic liver metabolism towards normal by insulin administration.

Glucagon-free insulin was administered to diabetic rats in doses sufficient to depress the blood glucose level to normal within 6 to 12 hours. Animals were sacrificed 10 minutes, 1, 6, 24 and 48 hours after the beginning of insulin administration and livers and diaphragms were removed. The livers were sliced and incubated with $\mathrm{C}^{\mathrm{1}}$ labelled glucose, fructose, glycerol, pyruvate and acetate. Substrate utilization, total glucose phosphorylation, substrate incorporation into glycogen, into glucose and into $\mathrm{CO}_{2}$ were measured. The marked differences between normal and diabetic liver metabolism were again demonstrated. In addition, it was found that whereas in diaphragm insulin exerts maximal effects within $10 \mathrm{~min}$ utes to 1 hour after its administration, in hepatic tissue insulin effects on all measurements did not begin until 6 hours, normal values being reached between 24 and 48 hours only.

This observation accounts for the inability to demonstrate effects of insulin added to liver in vitro, and further emphasizes the probable complex nature of insulin action. Possible implications as to treatment of diabetic acidosis will be discussed.

Protein-Binding of Thyroxine in Normal and Nephrotic Serum. J. Robbins, J. E. Rall and M. L. Petermann, New York, N. Y. (Introduced by R. W. Rawson).

The thyroxine in normal human serum is bound largely to a specific protein with electrophoretic mobility intermediate between alpha-1 and alpha-2 globulin (barbital buffer, $\mathrm{pH}$ 8.6). The thyroxine-binding capacity of this specific protein was studied in five normal serums. Synthetic 1-thyroxine-I ${ }^{200}$ was added in vitro in concentrations from 0.01 to 10 micrograms per ml.; and the distribution 
of radioactivity among the proteins separated by paper electrophoresis was determined. The pattern of distribution changed very little until the total serum thyroxine content reached approximately two to three times normal. At higher concentrations, thyroxine was found in increasing amounts in all protein fractions, but albumin became the major carrier. Essentially all of the thyroxine was bound to protein at even the highest concentrations, as shown by ultracentrifugation. Exchange between endogenous and exogenous thyroxine was demonstrated by the addition of unlabeled thyroxine to serum containing endogenously labeled thyroxine.

The derangement in iodine metabolism in the nephrotic syndrome includes a low serum organic iodine level, and a low BMR which is unresponsive to thyroxine administration. The serum thyroxine-binding protein in three patients with nephrosis was studied as described above. At low concentrations, thyroxine was bound largely to protein resembling the normal thyroxine carrier in electrophoretic mobility and in ultracentrifugal sedimentation. Excessive amounts of thyroxine, however, were carried to a large extent in the beta globulin, and the amount associated with albumin varied directly with the albumin concentration. The thyroxine-binding capacity of the specific protein, while possibly somewhat below normal, was adequate to bind at least the normal serum thyroxine content. A reduction in this protein does not, therefore, appear responsible for the lowered serum iodine in nephrosis.

\section{Effects of Potassium on the Renal Tubular Reabsorption} of Bicarbonate. KathleEN E. RoBERTS, HENRY T. Randall and Harold Sanders, New York, N. Y. (Introduced by Thomas P. Almy).

The renal reabsorption of bicarbonate and the excretion of titratable acid, potassium, phosphate, and ammonium have been studied in human subjects during metabolic (hypokalemic) alkalosis, and in dogs a) during respiratory acidosis and b) following acute reductions in glomerular filtration rate. Each patient was studied a) during a five-day control period, b) during hypokalemic alkalosis, and c) following administration of potassium. Calculations of the amount of base-bound bicarbonate reabsorbed per $100 \mathrm{cc}$./glomerular filtration rate shows that the hypokalemic patients reabsorbed 3.2 to $3.4 \mathrm{mEq}$./ $100 \mathrm{cc}$./glomerular filtration rate, as compared to the normal reabsorption of 2.7 to $2.9 \mathrm{mEq} . / 100 \mathrm{cc}$. of GFR. Following the administration of potassium, the bicarbonate reabsorption returns to normal coincident with correction of the plasma alkalosis. During the hypokalemic period, the urinary excretion of titratable acid, ammonium, and potassium decreased in comparison to the control period. Quantitatively, the increase in absorption of bicarbonate bound base could not be accounted for by the renal tubular exchange of hydrogen, ammonium, or potassium ions for sodium.

Experiments were carried out on dogs to determine the effects of potassium on the increased bicarbonate reabsorption: a) as described in conjunction with respiratory acidosis and which has been attributed to elevation of plasma $\mathrm{pCO}_{2}$, and b) as reported following acute reductions in glomerular filtration rate. In both situations the administration of potassium decreased the renal tubular reabsorption of bicarbonate to normal values. These studies and data to be discussed suggest that alterations in intracellular $\mathrm{pH}$ consequent to changes in intracellular concentrations of potassium, bicarbonate and $\mathrm{pCO}_{2}$ condition the renal reabsorption of bicarbonate in situations of hypokalemic alkalosis and respiratory acidosis.

\section{Affinity of Gentisate for Connective Tissues Demonstrated by Fluorescence in the Ultraviolet. BETTY S. Roof and JosepH C. TURner,* New York, N. Y.}

A part of the problem of the mode of action of antirheumatic drugs concerns whether they may be actually bound by connective tissues or exert their action less directly. Fluorescence microscopy has been applied by others to the study of the distribution in vivo of a number of aromatic compounds, and the claim has been made that salicylate "lacks connective tissue affinity." However, a systematic study of the distribution of antirheumatic substances appears to be wanting.

It was decided to examine the behavior of gentisate, because 1) gentisate has a far greater intensity of fluorescence than other antirheumatic agents, 2) it has relatively low toxicity, and 3) salicylate is oxidized in part to gentisate in the body and this conversion may indeed be essential to its action.

Mice and rats were injected intravenously with solutions of gentisate and sacrificed a few minutes later. When viewed with the eye in ultraviolet light, intense fluorescence was shown by the connective tissues such as tendon, cartilage, and subcutis. Viscera and muscle fluoresced dimly or not at all.

The technique can be refined by the application of ultraviolet microscopy. Meanwhile, the finding indicates that at least one active antirheumatic drug has an affinity in vivo for connective tissue. It would appear that an extension of the studies might provide useful information about certain aspects of the metabolism of these compounds.

For example, it has been possible to show in vitro that fragments of cartilage or tendon immersed in a watery solution of gentisate for $\mathbf{3 0}$ minutes exhibit fluorescence that persists after washing under the tap or after standing overnight in physiological saline. This indicates that the fluorescence observed in vivo is due to binding of gentisate by some element of connective tissue.

The Arresting Effect of Heparin Upon the Development
and Course of Experimental Nephrosis. RAY H. Rosenman, Sanford O. Byers and Meyer Friedman,* San Francisco, Calif.

The injection of rabbit anti-rat kidney serum into the rat induced a nephrotic state, characterized, as in man, by albuminuria, edema, ascites, lipemia, and hypercholesteremia. 
Administration of heparin was found to prevent or profoundly modify the development of this nephrotic syndrome if it were given either before or concomitantly with the injection of the anti-kidney serum. Thus, complete prevention of the syndrome was accomplished in a series of rats given a continuous intravenous injection of heparin immediately following the injection of potent anti-kidney serum and almost complete prevention, in three different series of rats given heparin intermittently following the administration of the specific serum. Thus the marked edema, ascites, lipemia and hypercholesteremia noted in the control injected animals were not observed in the rats also treated continuously with heparin. Serum injected rats given intermittent injections of heparin likewise failed to exhibit edema and ascites and showed only a minimal rise in serum lipids and cholesterol as compared to the control animals. The specific suppressive effect of heparin was demonstrated by the emergence of the full-fledged nephrotic state in the experimental rats 48 hours after discontinuance of the heparin therapy.

Administration of heparin to animals already exhibiting the nephrotic state also was found to depress markedly, their lipemia and hypercholesteremia. Possible therapeutic applications to clinical nephrosis will be discussed.

Plasma Thromboplastin Antecedent (PTA) Deficiency in Man: Clinical, Coagulation, Hereditary and Therapeutic Aspects. Robert L. Rosenthal, New York, N. Y. (Introduced by Arthur M. Fishberg).

In previous studies, an hereditary, hemophilia-like disease was discovered in 2 sisters and their maternal uncle who showed prolongations in the clotting time in the range of 20 to 30 minutes and abnormal prothrombin utilization. The clotting factor deficient in these cases, designated as plasma thromboplastin antecedent (PTA), was found to differ in properties from antihemophilic globulin (AHG) and plasma thromboplastin component (PTC). PTA is essential for the normal generation of thromboplastin during coagulation.

Studies on other members of the original family of PTA deficient patients revealed that PTA deficiency can also occur to a lesser degree characterized by a normal clotting time, normal to moderately prolonged heparin clotting time and impaired prothrombin utilization. These studies also established that PTA deficiency is transmitted by or to either male or female as a simple autosomal dominant trait.

Eighteen patients in 12 families ( 10 females, 8 males) have so far been diagnosed as PTA deficient. Except for the original 3 cases with prolonged clotting times, the clotting times in these patients have been normal. Many of these patients have been previously diagnosed as having either thrombasthenia or vascular defects. Hemorrhagic manifestations in these cases usually follow surgery or trauma and seldom occur spontaneously. Severe hemorrhage often follows tooth extractions and tonsillectomy. Purpura and joint involvement rarely occur. The administration of stored, refrigerated plasma has been found to correct the clotting defect and is effective in controlling hemorrhage or as a prophylactic measure prior to surgery.

These studies are significant in that many instances of unexplained, abnormal bleeding can now be attributed to PTA deficiency and can be effectively treated or prevented.

Auscultatory Determination of Arterial Pressure at Wrist and Ankle. David A. Rytand * and Samuel H. Boyer, IV, San Francisco, Calif.

Because the usual clinical methods yield erroneously high values for arterial pressure when the standard cuff is applied to large extremities such as thighs, use of a wider cuff has been recommended. But wider cuffs are not always available, nor has optimal width been established. We have found that the standard cuff is satisfactory on the legs, as well as on the arms of even grossly obese subjects, when it is applied just proximal to ankle or wrist. Auscultation at the appropriate artery is facilitated by removal of the plastic portion of the stethoscope bell. The method is practicable in all but hypotensive patients with faint Korotkow sounds.

In 53 recumbent normal adults, average systolic pressure by auscultation was $6 \mathrm{~mm}$. $\mathrm{Hg}$ lower in the radial than in the brachial artery, mean diastolic pressure (muffling) $6 \mathrm{~mm}$. $\mathrm{Hg}$ higher; the discrepancy was less than $20 \mathrm{~mm}$. $\mathrm{Hg}$ in $90 \%$ of observations (not quite simultaneous). In the posterior tibial and dorsalis pedis arteries, systolic pressure was $2 \mathrm{~mm}$. $\mathrm{Hg}$ and diastolic pressure $5 \mathrm{~mm}$. Hg above brachial levels; these values resembled those at the popliteal artery distal to a wider cuff at the thigh.

In 20 hypertensive patients, mean pressure relationships were as above, with greater individual variations. With aortic insufficiency, findings were similar but for Hill's elevation $(30 \mathrm{~mm} . \mathrm{Hg})$ of posterior tibial systolic pressure. Discrepancies between muffling and disappearance of sound at diastolic levels were 3 times greater at the brachial than at the radial artery. In 3 patients with mammoth obesity, radial and ankle pressures were far below brachial ones $(60-140 \mathrm{~mm}$. $\mathrm{Hg}$ systolic, $20-85 \mathrm{~mm}$. $\mathrm{Hg}$ diastolic); this was not so with obesity of ordinary degree. Results following operation for coarctation of the aorta were readily followed.

\section{Clinical, Metabolic and Genetic Studies in Myotonia} Dystrophica. AvERY A. SANDBERG, FayETTE E. Strphens and Frank H. Tyler,* Salt Lake City, Utah.

In personally examining the members of 15 kindreds in which myotonia dystrophica was found more than 100 affected individuals were observed. Nearly all were residents of the local rather small population, indicating that this disease is among the most common of neuromuscular disorders in this locality. Although the disease showed a wide spectrum of expression, in most cases the involvement was rather typical. The following features were usually present: myotonia, muscular atrophy in a characteristic distribution, subcapsular cataracts, gonadal in- 
sufficiency, frontal alopecia, and peripheral trophic and vasomotor changes, sometimes with Raynaud's phenomenon. A high proportion of the patients had cardiac dystrophy. Progress of a barium meal through the intestine was frequently slow and irregular. Absorption of certain substances (glucose, iron) was delayed. These changes are probably the result of involvement of smooth muscle. The incidence of mental deficiency was greater than in patients with other muscular dystrophies or in the general population.

In 17 of these individuals detailed metabolic and endocrine studies were performed. Because certain of the clinical features resemble myxedema and basal metabolic rates are low, others have suggested that hypothyroidism is an etiological factor in the disorder. We have demonstrated normal $I^{181}$ uptake by the thyroid gland in these patients; the low metabolic rate is probably due to the loss of muscle mass. Although in contrast to other muscular dystrophies, creatinuria was irregular in these cases of myotonia dystrophica, serum creatine levels were usually elevated. Adrenocortical function was normal, although the 17-ketosteroid excretion in the urine was low in patients with testicular atrophy.

Inheritance of the trait was consistent with that of an autosomal dominant. Although penetrance appeared to be complete there was an extremely wide range of expression of the trait.

Antibody Synthesis in Vitro. Jay P. Sanford, Cutting

B. Favour and Ericka Lindsay, Boston, Mass. (Introduced by John G. Gibson, II).

The demonstration of a humoral immune factor by Pfeiffer and Marx in 1898 and the implication of the spleen playing a major role in its production stimulated numerous investigations concerning the sites of antibody synthesis. The present studies were undertaken to obtain further data on the role of the circulating lymphocyte in relation to that of splenic cells in the immune response. The antigen used was the enzyme urease. Antibody (anti-urease) was measured by the inhibition of ammonia and of carbon dioxide release from a urea substrate by a known quantity of urease. The injection of this material into rabbits produced high levels of circulating antibody.

The determinant group in the urease to which the antibody appears directed is not that portion of the molecule which is responsible for its enzymatic action, as demonstrated by the poor "anti-urease" activity where the specific antigen-antibody complex was not removed from solution by centrifugation.

Our data clarify further the role of the circulating lymphocyte and its relationship to splenic cells in the immune response. Both the spleen and circulating lymphocytes from an immune rabbit contain a quantity of specific antibody which is released upon incubation in vitro. Cellular respiration may be inhibited by toluol without affecting this release of antibody. An additional quantity of antibody is produced by splenic tissue in vitro if metabolic activity is allowed to continue. In contrast the lymphocyte, while metabolically active, is unable to increase the amount of antibody released in vitro.

This circulating antibody is produced in association with plasmacytic maturation, at least within the spleen. The circulating lymphocyte may carry the antibody and be associated with its release; however, it does not have the capacity for antibody synthesis in vitro.

Solute Load and the Renal Osmotic Parameters of Chronically Dehydrated Men. F. SARGENT, II and R. E. Jornson,* Urbana, IIl.

Urine flow and urine-serum osmolar ratio (U/S) are quantitatively related so that the minimum flow at a given solute load is the asymptote for maximal $U / S$ ratios. This parameter has been used repeatedly in discussions of water requirements, to the neglect of another measurement that is important in renal function: the urine flow at which $U / S$ becomes 1.0 . The first parameter defines the condition for maximal renal osmotic work; the second, that for minimal work. Male students, apparently free from disease, were subjected during two week periods in a comprehensive metabolic study of five months' duration to all possible combinations of the following average daily allowances: water, unlimited or 0.9 liters; sodium chloride, 0,5 or 20 grams; nitrogen, $0,6,12,17$ or 24 grams; and calories, $0,1000,2000$ or 3000 . Chronic dehydration combined with high solute load resulted in daily urine flows of about 0.8 liters as a minimum, and U/S osmolar ratios as high as 5.9 ; in contrast, when solute load was low (pure carbohydrate or high fat diet) minimum daily urine flow was about 0.25 liters and maximum U/S about 4.2. With unlimited water and high solute load, the U/S ratio approached 1.0 as an asymptote at daily flows of 2.5 liters or over; in contrast, with unlimited water and a low solute load U/S reached 1.0 with daily urine flows of 0.8 liters and then approached an asymptote of less than 0.1 with greater flows. We conclude that the urine flow for minimal renal osmotic work increases with increasing solute load and is related importantly to water requirements and renal function during different dietary regimens.

[Work done under Contract AF 18(600)-80 from Air Research and Development Command.]

\section{Motion Picture Studies of Ventricular Muscle Dynamics in Experimental Localised Ischemia, Correlated with Myocardial Oxygen Tension and Electrocardiograms. John J. Sayen, Warner F. Shridon, Grorge Peirce and Peter T. Kuo, Philadelphia, Pa. (Introduced by Charles C. Wolferth).}

It is well known that systolic bulging of muscle occurs in the distribution of an acutely occluded coronary artery. Disturbed muscle contraction is an essential index of severe myocardial ischemia. Acute localized ischemia was studied in dogs by continuous recording of polarographic oxygen tension and simultaneous intramyocardial electrocardiograms from ten platinum electrodes inserted in the left ventricle to various depths. The relative motions of these electrodes could then be analyzed from motion picture records by measuring the distances between 
markers attached to their glass shafts at the epicardial surface. Events were synchronized by a flashing neon bulb, triggered by the first upward deflection of each QRS complex in an intramyocardial electrocardiogram. Color film permitted rough estimations of epicardial arteriovenous differences and muscle discoloration.

Results: Non-ischemic hearts yielded various types of muscle contraction record. The areas of earliest shortening reduced presystolic muscle segment lengths by as much as $1 / 6$ within 0.25 seconds of electrical systole. Other areas showed delayed shortening or very slight changes. Regardless of its control contraction pattern, muscle in severely ischemic areas was stretched rapidly just after electrical systole, reaching a maximum length increase about 0.25 second thereafter. Less severely ischemic muscle failed to shorten or stretched after partial systole. Muscle beyond the ischemic area was unaffected or somewhat increased its amplitude of contraction.

Coronary occlusion produced rapid falls of oxygen tension beginning within six to eight heart beats. Muscle contraction disturbance could be detected as early as the eighth heart beat. An extrasystole regularly occurred within fifteen heart beats and about this time the intramyocardial electrocardiogram began to show abnormality. Epicardial electrocardiograms became abnormal later, often not until oxygen tension had fallen profoundly. Very severe acute coronary narrowing resembled occlusion in its consequences. Slighter narrowing could cause reduced oxygen tension and intramyocardial electrocardiographic abnormality without measurably changing epicardial electrocardiograms or muscle contraction.

The Concentration of Copper and Ceruloplasmin in Maternal and Infant Plasma at Delivery. I. Herbert Scheinberg, ${ }^{*}$ Charles D. Cook and Janet A. Murpey, New York, N. Y.

At delivery, maternal plasma has a concentration of copper about 5 to 6 times that of infant plasma. Almost all of the copper in the plasma of normal adult human beings is irreversibly bound within the protein molecule, ceruloplasmin, whose molecular weight is 150,000 . It seemed unlikely that equilibrium with respect to diffusion across the placental membrane would be approached by a molecule of this size, whereas non-ceruloplasmin copper might be expected to be in such equilibrium. This study was undertaken to investigate these suppositions.

The concentrations of total copper, of ceruloplasmin and of non-ceruloplasmin copper were measured in maternal plasma, taken immediately before delivery, and in plasma from the umbilical cord. In twelve pairs of mothers and infants, the average level of total copper in maternal plasma was 216 (range: 118-302), and that in cord plasma was 36 (range: $12-67$ ) gamma/100 ml. In the same subjects, the average level of ceruloplasmin in maternal plasma was 55.6 (range: $39.4-89.0$ ), and that in cord plasma was 6.5 (range: 1.8-13.1) $\mathrm{mg} . / 100 \mathrm{ml}$. Since $0.34 \%$ of ceruloplasmin is copper, the maternal plasma contained an average of 189 and the cord plasma contained an average of 22 gamma/100 ml. of ceruloplasmin copper. In fifteen pairs of mothers and infants the average levels of non-ceruloplasmin copper were 10.7 (range: 5-15) and 8.5 (range: 0-16) gamma/100 ml. for maternal and infant plasma, respectively. Non-ceruloplasmin copper appears to approach equilibrium with respect to diffusion across the placenta since the average difference between maternal and infant values is not statistically significant by the usual criteria. In contrast, the fact that ceruloplasmin copper is not in such equilibrium accounts for the large gradient of copper between maternal and infant plasma.

Neurogenic Factors in Coronary Artery Syndromes. I. Experimental Production of Electrocardiographic Patterns Simulating Acute Myocardial Infarction, Coronary Insufficiency and Cardiac Arrhythmias by Stimulation of Autonomic Nerves. SidNEy Scherdrs, R. Adams Cowley and George Schimert, Baltimore, Md. (Introduced by Theodore E. Woodward).

Psychogenic factors are known to precipitate the anginal syndrome of coronary insufficiency although the nervous pathways have not been clearly defined. Furthermore, thrombosis of the coronary arteries and ultimate myocardial infarction are said to result from arteriosclerotic changes, yet the factors culminating in the acute episode have not been clearly elucidated. Based upon well founded clinical considerations, we accepted the premise that neurogenic factors may precipitate episodes of coronary insufficiency and even occlusion in patients with predisposing atherosclerosis. The dog has been employed as an experimental model to investigate these factors.

After transthoracic thoracotomy, the left vagus and sympathetic nerves were exposed and stimulated at various sites by faradic current. Uniform electrocardiographic changes simulating patterns typical of acute myocardial infarction occurred promptly when isolated vagal branches to the cardiac plexus were stimulated, viz.: elevation and coving of ST segments and $T$ wave inversion in certain conventional leads. Less frequently, ST segments were depressed and $T$ waves became sharper with increased amplitude simulating the pattern of acute coronary insufficiency. Stellate ganglion stimulation likewise produced the latter type of electrocardiographic response, and minimized the electrocardiographic effects of preceding "vagal" stimulation. Stimulation of vagal cardiac nerve pathways resulted in cardiac arrhythmias : auricular flutter-fibrillation, A-V nodal rhythm, extrasystoles, ventricular standstill, tachycardia and fibrillation. On one occasion, following brief stimulation, an "acute coronary-like" pattern was noted, followed immediately by ventricular fibrillation and death.

These striking electrocardiographic changes hitherto unreported following autonomic nerve stimulation suggest either 1) interference with coronary blood flow or 2) alteration of myocardial function by metabolic mechanisms.

These preliminary findings are discussed relative to theories of pathogenesis of coronary occlusion. Blocking 
action by pharmacological methods has received consideration.

A new surgical approved therapy of coronary disease based upon these factors is under investigation.

Erythropoietic (Congenital) Porphyria: $A$ Blood Dyscrasia Characterized by a Peculiar Disturbance of the Normoblasts. RUdi Schmid, Samuel Schwartz and R. Dorothy Sundberg, Minneapolis, Minn. (Introduced by C. J. Watson).

Recent studies have shown that the photosensitive forms of porphyria include two fundamentally different disturbances of porphyrin metabolism, one related to hemoglobin formation during erythropoiesis (Porphyria erythropoietica), the other a liver dysfunction (Porphyria hepatico). Erythropoietic (congenital photosensitive) porphyria is a rare inherited disease manifest at birth or during early life. Critical review of the reported cases in the literature reveals only 34 instances, in which the diagnosis could be established beyond reasonable doubt. They were 19 female and 15 male children, the great majority having erythrodontia, hypertrichosis, hemolytic anemia and splenomegaly.

Bone marrow and blood preparations of five of these patients, were available for study. In all instances, excessive amounts of porphyrins were present in marrow and circulating erythrocytes. Using fluorescence microscopy, most of the porphyrin was found in the nuclei of the normoblasts in the marrow. By studying and photographing the same group of marrow cells, first in the fluorescence microscope, then with absorption spectroscopy and, finally, by using a specialized staining technique, the existence of a normal and an abnormal line of normoblasts was discovered. Cells of the abnormal variety exhibit high concentrations of porphyrins in their nuclei as well as characteristic intranuclear inclusions, containing hemoglobin. This, in all probability, represents the inborn metabolic error of this disease. The normal cell type shows neither porphyrin fluorescence nor nuclear inclusion bodies.

The ratio of normal to abnormal normoblasts in the bone marrow varies in different patients from $1: 3$ to $3: 1$, but it does not appear to be related to the severity of the clinical manifestations. Splenectomy performed in 3 cases did not alter this ratio, but it did correct the hemolytic anemia. As a result, the normoblastic hyperplasia and hence porphyrin formation and excretion decreased.

The Relation of Life Situations to Metabolic Balance. W. W. Schotrstaedr, Oklahoma City, Okla. (Introduced by Stewart Wolf).

Three adult males without known renal or cardiovascular disease have been studied under rigid control on a metabolic ward for periods up to eight weeks in duration. Records were kept of events occurring on the ward and of the attitudes and emotions or feeling states aroused in the patients by these events. No stress interviews or contrived situations were introduced. The subjects were maintained on uniform intake of water, electrolytes and diet throughout the period of study and daily determinations of urinary nitrogen, electrolytes, and water excretion were made.

Marked variations in rates of excretion were observed despite the rigid regimen. In a subject on whom urine specimens were obtained four times a day, the evening urine volumes varied from 0.49 to $2.88 \mathrm{cc} / \mathrm{min}$; sodium excretion from 34 to $278 \mu \mathrm{Eq}$./min; potassium from 52 to $182 \mu \mathrm{Eq} . / \mathrm{min}$; nitrogen from 3.23 to $13.83 \mathrm{mg} / \mathrm{min}$; and corticoids from 0.35 to $1.53 \mu \mathrm{Eq} . / \mathrm{min}$ despite constant intake and constant exercise. 24 hour urine volumes varied from 630 to $1680 \mathrm{cc}$ and urinary nitrogen varied from 7.02 to $13.00 \mathrm{gms}$. All marked variations from the average rates of excretion were easily correlated with troublesome events occurring spontaneously. For example, anxiety was induced by the illness of other patients and anger because a meal was served cold instead of warm. Tension was induced by brooding over past health and depression because of the failure of expected visitors to appear.

The study has shown that careful control of intake and activity is not adequate to assure a steady metabolic state. Marked variations in urinary excretion of nitrogen, electrolytes and water correlated with events occurring during ward routine and with the emotions and feeling states aroused by these events.

The Osmotic Activity of Cells of Mammalian Tissues. Irving L. Schwartz and Eugene L. OpIe, New York, N. Y. (Introduced by Vincent P. Dole).

Slices of liver and kidney cortex, taken from rats immediately after death and immersed in a series of salt solutions, swell if the concentration is less than about twice that of extracellular fluid, and shrink if the concentration is greater. From these results it is inferred that the osmotic activity within the cells of the slice is about twice that of the solutes in normal extracellular fluid.

This conclusion, however, depends on the assumption that salt remains extracellular during the period of measurement. Evidence to support this assumption has been obtained in the following experiments : fresh slices of liver, kidney cortex, and corium were weighed, immersed for periods of time from 1 to 60 minutes in solutions of 0.1 to $1.0 \mathrm{~N} \mathrm{NaCl}$, reweighed to measure the gain or loss of water, and then analyzed for electrolytes. It was found that the entry of sodium into the tissues was independent of the movement of water, and simply proportional to the concentration of sodium in the external medium. The results indicated that the medium quickly came into equilibrium with $20 \pm 2 \%$ of the liver mass, $31 \pm 2 \%$ of the kidney, and $49 \pm 1 \%$ of the corium-values which are close enough to those accepted for extracellular space to suggest that the effect of immersion within the first ten to fifteen minutes is to equilibrate the bath and the interstitial fluid. Later, the intracellular concentration of sodium rises, but at the end of the first ten minutes only the interstitial fluid has equilibrated. Therefore, the isosmotic solution can be taken as a measure of the osmotic activity of cells in the tissue slices. 
Effect of Cortisone on Intracellular Peptidase Activity. Theodore B. Schwartz, Durham, N. C. (Introduced by James V. Warren).

Dipeptidase activity of surviving diaphragm has been used as a model to represent intracellular protein catabolic processes. This enzymic activity is determined by measuring the rate at which glycylglycine is hydrolyzed by viable isolated rat diaphragm tissue. The enzyme is strongly activated by cobalt and inhibited by cysteine, and these variations have been demonstrated both in vitro and in vivo. A $50 \%$ increase in dipeptidase activity of surviving diaphragm followed the administration of cortisone, $5 \mathrm{mgm}$. a day, over a 5-day period. This hormonal effect requires the presence of intact cells since it was not demonstrable in rat muscle extracts or homogenates. The mechanism by which such biologic activation occurs is being explored; and there are indications that this cortisone-induced enhancement of peptidase activity of isolated diaphragm is not mediated by way of increased availability of cobalt ion since, when the experiment was repeated with optimal amounts of cobalt added in vitro, dipeptidase activity of cortisone-treated animals remained greater than normal.

These observations are consistent with the view that the protein catabolic effect of adrenal steroids is, in part, the result of enhanced intracellular, proteolytic activity. This hormonal effect is demonstrable only in the presence of viable, intact cells and apparently does not operate through metal activation.

The Dependence of Renal Sodium Reabsorption on $H y$ drogen Exchange. William B. SchWARTz * and Arnold S. Relman, Boston, Mass.

Net tubular transport of water and electrolytes has been measured in well-hydrated anesthetized dogs given large doses ( $500 \mathrm{mgm} . / \mathrm{kgm}$.) of acetazoleamide, a potent carbonic anhydrase inhibitor. Frequently, half or more, and on several occasions two thirds, of the filtered sodium was excreted together with eighty to ninety per cent of filtered bicarbonate, twenty to forty per cent of filtered chloride and an amount of potassium at least equal to, and up to, eighty-six per cent greater than the filtered load of potassium.

During this brief profuse diuresis, urine volume was one-half to two-thirds of the volume of the glomerular filtrate and $U / P$ osmolar ratios approximated unity. Filtration rate was usually unaffected or slightly reduced.

Maximal depression of bicarbonate reabsorption (to rates of .1 to $.6 \mathrm{mEq}$. $/ \mathrm{min} . / 100 \mathrm{cc}$. GFR) was observed with large doses of the inhibitor at widely varying levels of plasma bicarbonate (19-75 $\mathrm{mEq} . / \mathrm{L}$.) and blood $\mathrm{pCO}_{2}$ $(25-100 \mathrm{~mm} . \mathrm{Hg})$. With lower doses of inhibitor (10$50 \mathrm{mgm} . / \mathrm{kgm}$.), one-third to one-half the normal load of bicarbonate can be diverted into the urine with little or no consistent effect on chloride excretion; the latter was consistently increased only with maximal dosage.

These observations are consistent with the hypothesis that all bicarbonate reabsorption is accomplished by tubular exchange of hydrogen for sodium and that carbonic anhydrase is required to catalyze this reaction if the rate of reabsorption is to be more than minimal. The data further suggest that most, if not all, sodium reabsorption is effected by hydrogen-sodium exchange. The large increment in chloride excretion seen only with maximal doses of inhibitor is probably secondary to the diuresis of sodium which results when the rate of tubular hydrogen ion generation falls significantly below the rate of filtration of sodium chloride.

Potassium Intoxication During and Immediately Following Respiratory Acidosis. Belding H. SCRIBNER, George M. Bogardus, Kenneth Fremont-Smith and James M. Burnell, Seattle, Wash. (Introduced by Robert S. Evans).

Unexplained cardiac arrest and death have been observed during and immediately following the respiratory acidosis of general anesthesia. These studies indicate that potassium intoxication associated with respiratory acidosis may be an important factor.

Dogs were made acidotic for 2-5 hours with a mixture of $30 \% \mathrm{CO}_{2}$ and $70 \%$ oxygen. Blood $\mathrm{pH}$ fell promptly and remained between 6.7 and 6.9. Balance studies revealed that during the acidosis 5 to 15 milliequivalents of potassium were transferred per hour from the cells into the extracellular space. Arterial serum potassium always rose at a rate which varied inversely with urinary excretion. Most animals survived serum potassium levels as high as 9 to $10 \mathrm{mEq}$./L. without electrocardiographic changes as long as they were maintained in acidosis. Immediately upon return to room air, blood $\mathrm{pH}$ rose abruptly and electrocardiographic evidence of potassium intoxication appeared often progressing to cardiac arrest and death. Prompt reinstitution of respiratory acidosis was frequently lifesaving.

The appearance of potassium intoxication with the relief of respiratory acidosis was related to at least 2 factors: 1) There was a transient further increase in the serum potassium level of 0.5 to $1.0 \mathrm{mEq}$./ $\mathrm{L}$. when the acidosis was terminated. 2) Potassium loading studies revealed that potassium intoxication in non-acidotic dogs occurred at serum concentrations 2 to $4 \mathrm{mEq}$./L. lower than in dogs in respiratory acidosis.

These data suggest that certain unexplained anesthetic deaths may be due to potassium intoxication because: 1) Respiratory acidosis causes hyperkalemia. 2) Relief of acidosis causes further transient hyperkalemia. 3) There is less tolerance to hyperkalemia at normal blood $\mathrm{pH}$ than during respiratory acidosis.

The Relation of Hypokaliemic Alkalosis Induced by Adrenal Steroids to Renal Acid Secretion. Domald W. Seldin,* Floyd C. Rector, JR., Norman Carter and John Copenhaver, Dallas, Tex.

The cause of the alkalosis which develops in animals rendered potassium deficient by administration of DOCA and sodium has not been clearly elucidated. To clarify the role of the kidneys in the alkalotic process, rats were tube-fed standard electrolyte-deficient diets for 14 days 
and treated with various combinations of electrolytes and DOCA. Urine was analyzed for $\mathrm{pH}$, titratable acid, and ammonium. Muscle and serum electrolytes were measured. Activities of glutaminase, glycine oxidase, leucine oxidase, and carbonic anhydrase were determined in the kidneys.

Rats receiving sodium, DOCA, but no potassium, developed severe systemic alkalosis and excreted approximately 8 meq. more acid, primarily as $\mathrm{NH}_{4}$, than corresponding control animals. The alkalosis must therefore be, in part at least, a consequence of the renal loss of acid. Associated with this accelerated ammonium excretion renal glutaminase activity increased from $788 \pm 39 \mu \mathrm{M} \mathrm{NH}_{3} /$ $100 \mathrm{mgm}$. dry kidney/hr. in control animals to $1842 \pm 445$ in animals rendered alkalotic with sodium and DOCA. Neither renal carbonic anhydrase nor ammonia-producing enzymes other than glutaminase changed significantly with increased acid secretion.

The increased glutaminase activity was not a consequence of any direct action of DOCA or sodium on the renal tubules, since rats given either DOCA or sodium salts, but with sufficient potassium to prevent potassium deficiency, developed neither alkalosis nor increased glutaminase activity.

These findings suggest that hypokaliemic alkalosis produced by DOCA and sodium is, in part at least, a consequence of the renal loss of acid, principally as ammonium. Accelerated ammonium excretion, in turn, apparently results from the activation of renal glutaminase. This enzymatic adaptation was not a result of the direct effect on the tubule of sodium or DOCA, but occurred only if potassium deficiency supervened. It is suggested that the adaptation of glutaminase may be stimulated either by potassium depletion or intracellular acidosis.

Triiodothyronine Administered Orally in the Treatment of Myxedema. Herbert A. Selenkow and Samuer P. ASPER, JR.,* Baltimore, Md.

Recent investigations indicate that triiodothyronine is the most potent form of the thyroid hormone known. The levo isomer of triiodothyronine, administered parenterally to patients with myxedema, possesses three to four times the calorigenic effect of $l$-thyroxine and exhibits all the metabolic properties ascribed to the thyroid hormone.

In the present study the calorigenic activities of $l$ triiodothyronine, $d l$-triiodothyronine and sodium $l$-thyroxine administered orally were determined in six patients with myxedema. The daily dose required to maintain euthyroidism was 70 to 105 micrograms of $l$-triiodothyronine, 200 to 300 micrograms of $d l$-triiodothyronine and 300 micrograms of sodium l-thyroxine. All patients receiving triiodothyronine became clinically euthyroid within ten to fourteen days. During this period each patient exhibited a six to ten pound loss in weight, a decrease in the serum cholesterol to normal levels and an increase in basal metabolic rate to normal values. In addition, during administration of either $l$ or $d l$-triiodothyronine, levels of serum protein-bound iodine were in the range of myxedema at times when the patients were completely euthyroid. This has also been noted following parenteral administration of triiodothyronine. It is apparent that the level of the serum proteinbound iodine is not indicative of the metabolic status of athyreotic patients receiving triiodothyronine as judged by present standards of the normal range for this determination.

Triiodothyronine administered orally was uniformly effective in alleviating myxedema. The $d l$-isomer of triiodothyronine had approximately one-half the calorigenic effect of an equivalent dose of $l$-triiodothyronine; this suggests that the $d$-isomer probably is calorigenically inactive. The $l$-isomer of triiodothyronine possessed three to four times the calorigenic effect of sodium $l$-thyroxine. Triiodothyronine has the therapeutic property of acting rapidly in minute doses. Like thyroxine, it is a chemically pure synthetic form of the thyroid hormone, obviating standardization.

The Participation of Fibrin in the Activation of the Fibrinolytic Ensyme of Plasma. Sol SHarry,* Cincinnati, $O$.

A number of observations have suggested that, in native plasma, under conditions conducive to the activation of plasminogen (the precursor of the fibrinolytic enzyme), fibrinolysis proceeds at a much faster rate than fibrinogen breakdown. However, plasmin, the active enzyme, in purified systems attacks fibrinogen and fibrin at the same rate and its activity on these substrates appears to be directly related to its general proteolytic properties. To explain this paradox, Ratnoff has suggested an accelerated activation of plasminogen in the presence of a fibrin clot.

We have obtained direct evidence, in vitro, that the streptokinase activation of plasminogen in plasma is markedly accelerated by the presence of a fibrin clot. The observations indicate that activation rapidly occurs on the fibrin where considerable amounts of plasminogen and streptokinase are adsorbed. The local activation of the enzyme on the fibrin results in rapid fibrinolysis, followed by release of the activated enzyme into the surrounding plasma, where it is slowly inhibited. In vivo observations, following the intravascular injection of streptokinase into dogs with intraarterial thrombi resulted in a very small increase in circulating proteolytic activity and minor changes in the plasminogen and fibrinogen concentrations of the plasma, yet produced rapid liquefaction of normally clotting blood specimens, and significant dissolution of the experimental thrombi. In sharp contrast, when plasminogen was completely activated in the plasma, in vivo, by another mechanism, large amounts of circulating proteolytic activity appeared, the plasminogen disappeared, and an incoagulable state rapidly resulted. Dissolution of the experimental thrombi, however, was similar to the streptokinase treated animals.

The observation that fibrin provides a favorable surface for the mediation of the activation of plasminogen by streptokinase suggests that this phenomenon may be in- 
volved in naturally occurring fibrinolysis, and may also explain the special fibrinolytic activity observed in patients following streptokinase injections.

The Mechanism of Pigmentation in Addison's Disease. Kazuo Shizume, Aaron Bunsen Lerner and Thomas B. Fitzpatrick, Portland, Ore. (Introduced by Arthur W. Frisch).

Clinical and experimental evidence will be presented to show that the hyperpigmentation of Addison's disease is probably due to an increase in the release of the melanocyte stimulating hormone (MSH) from the pituitary gland as a result of a decrease in adrenalcortical function.

Administration of $\mathrm{MSH}$ to four persons produced an increase in pigmentation. After discontinuation of the hormone, pigmentation gradually decreased, and the skin returned to its previous color.

Blood and urine levels of $\mathrm{MSH}$ are increased in Addison's disease. When cortisone given for treatment is withdrawn, urinary output of $\mathrm{MSH}$ is increased. The hyperpigmentation of Addison's patients, as well as the normal pigmentation of frogs, decreases following administration of cortisone. Cortisone probably inhibits the production of MSH by the pituitary gland.

Commercial ACTH, preparations contain fairly large amounts of MSH. The hyperpigmentation resulting from long-term treatment with ACTH may result from the action of MSH present as a contaminant in the ACTH preparation.

\section{Demonstration of Antivessel Agents in Serum of Patients} with Anaphylactoid Purpura and Periarteritis Nodosa. Mario Stefanini * and Iraca B. Mednicoff, Boston, Mass.

Two cases were observed with associated acute idiopathic thrombocytopenic purpura and Henoch-Schönlein's anaphylactoid purpura. In one, a platelet isoagglutinin could be detected. In view of the recently emphasized immunologic nature of idiopathic thrombocytopenic purpura, search was conducted for agents in the serum of these patients capable of reacting with vascular elements. Decalcified serum in both instances contained precipitin against stable, saline-soluble antigen prepared from aortic intima and from total aorta of newborn infants. Complement was fixed in the course of this reaction. With separation by means of paper electrophoresis in one case (Dr. Carlos Guzman L.) the active fraction was found to be $\gamma$-globulin. The agent was relatively labile during storage (inactivated after 10 days, even at freezing temperature), and destroyed at $60^{\circ} \mathrm{C}$.

A standard preparation of the aortic antigen was made, containing $20 \mathrm{mgs}$. N\%. With this antigen, decalcified sera gave positive precipitation in 6 out of 8 cases of anaphylactoid purpura, 3 out of 4 cases of periarteritis nodosa. Test was negative in acute idiopathic thrombocytopenic purpura (5 cases) and lupus erythematosus (3 cases). Antigens were prepared from intimal and from adventitial and elastic layers of aorta. Perhaps due to difficulties in separating various layers of the aorta, positive sera reacted equally with both antigens. Plasma from two patients with anaphylactoid purpura was injected into compatible normal individuals. No purpura, no positive tourniquet test developed, but the antivessel factor was transmitted to the recipient.

These results appear to indicate the role of circulating antivascular agents in the pathogenesis of vascular damage in anaphylactoid purpura and periarteritis nodosa. It is likely that these factors develop as immunologic response (antibodies), since in experimental animals one obtains antivessel serum by administration of heterologous aorta constituents.

Disappearance from Serum of $I^{12}$-Labeled L-Thyroxine and L-Triiodothyronine in Euthyroid Subjects. KeNneth Sterling, Joyce Lashof and Evelyn B. Man, New Haven, Conn. (Introduced by Philip K. Bondy).

Radioactively labeled 1-thyroxine or 1-triiodothyronine in tracer amounts was injected intravenously in nine euthyroid hospital patients and two normal volunteers. Serum butanol extractable iodines were normal.

Patients received an excess of non-radioactive iodine during studies to minimize thyroid uptake and reutilization of radioiodine liberated on degradation of the administered compounds.

Serum radioactivity was measured on blood samples drawn ten minutes after injection, and daily for two weeks. There was a rapid initial fall, attributed to a distribution phase. Within two days after injection of radiothyroxine, the serum radioactivity declined more gradually, assuming a straight line on semilogarithmic plot. This slow exponential component of the disappearance curve was interpreted as indicating thyroxine turnover, or the rates of formation and degradation, which in a steady state should be identical. The mean halftime of thyroxine turnover in six subjects was 6.5 days.

The disappearance curve observed after injection of labeled triiodothyronine was much more rapid, with a mean half-time of 2.6 days in four subjects. Successive studies with 1-thyroxine and 1-triiodothyronine in one individual clearly demonstrated the more rapid disappearance of the latter compound.

Studies on Hydralazine: Evidence for a Peripheral Site of Action. Albert Stunkard, Lothar Werthemmer, and Walter Repisch, New York, N. Y. (Introduced by David Seegal).

An attempt was made to determine the site of action of the antihypertensive agent, hydralazine, by measurements in man of blood flow in intact and denervated extremities. Control studies of 4 patients in whom the nervous system was intact established that intravenous administration of hydralazine caused either no change or slight decrease in skin temperature, and blood flow as measured by plethysmography of the limb. The legs of 4 patients with spinal cord transection, however, showed significant increases in both skin temperature and blood flow, while the upper extremities with intact innervation responded to hydralazine administration in the usual manner. 
When denervation of the limb was achieved by unilateral sympathectomy, hydralazine again increased blood flow to the denervated extremity (the leg) while reducing it in the intact leg. These observations suggested that hydralazine might act at the blood vessel wall itself. The possibility that the action might represent adrenergic blockade was excluded by the failure of hydralazine to decrease the hypertensive and bradycardic effects of norepinephrine infusion. Furthermore, no effect on the cold pressor response was observed following doses of hydralazine large enough to lower blood pressure. It was concluded that hydralazine could act directly upon the blood vessel wall, and that this might be a major site of action.

Catheterization Studies on the Bronchial and Pulmonary Vascular Systems in Dogs; With Special Reference to the Chemotherapy of Lung Cancer. R. D. Sullivan, T. C. Alford and D. A. Karnofsky, ${ }^{*}$ New York, N. Y.

Polyethylene catheters were introduced at thoractomy, singly and in combination, into the right bronchial artery and into various branches of the pulmonary arteries and veins. The catheters were brought out through the chest wall and remained in situ for periods as long as three months. By injecting Diodrast, serial angiograms were obtained under various experimental conditions in studies on 23 dogs. This catheterization technique may be applied to respiratory physiologic studies.

Dye injected into the bronchial or pulmonary arteries demonstrated the arborizations of these vessels, and was rapidly cleared. After ligating the pulmonary artery and vein to a lobe, dye injected into the lobe artery or vein pooled in the vessels of the occluded lobe; however, 3 days later, dye given in the same manner, filled the bronchial arterial tree, indicating that collateral channels had opened between the pulmonary and bronchial vessels. Additional collateral channels appeared between the bronchial artery and the systemic circulation. Thus, it is possible to distribute dye throughout the bronchial arterial system by ligating a pulmonary vein, and injecting dye into the pulmonary artery to the same lobe.

Parallel procedures were performed with nitrogen mustard $\mathrm{HN}_{2}, 1 \mathrm{mg} / \mathrm{kg}$, injected into the pulmonary and bronchial vessels. No local injury resulted from the injection of $\mathrm{HN}_{2}$ into the unaltered pulmonary artery, whereas introduction into the bronchial artery severely injured the entire tracheo-bronchial tree and hilar structures, including the lymph nodes. Similar effects were produced by injecting $\mathrm{HN}_{2}$ into the pulmonary artery three days following ligation of the pulmonary artery and vein. In contrast instillation of $\mathrm{HN}_{2}$ through a bronchoscope into a major bronchus produced edema, hemorrhage and focal necrosis in the pulmonary parenchyma only. Since lung cancers and their regional metastases derive their blood supply from the bronchial artery, methods for modifying the pulmonary circulation at exploratory thoractomy enhance the opportunities for the local chemotherapy of these neoplasms.
A Forty-Eight Hour Procedure for the Determination of Post-Transfusion Survival of Erythrocytes from Preserved Blood, Employing Radioactive Chromium-51 $\left(C r^{B 1}\right)$. Timothy R. Talbot, JR., Mary Sax and Alicr Cary, Philadelphia, Pa. (Introduced by Thomas Fitz-Hugh, Jr.).

The method herewith presented obviates the need for blood volume determination and requires only forty-eight hours to give an accurate measure of post-transfusion survival of erythrocytes.

We have found that 25 to $50 \mathrm{ml}$. of $\mathrm{Cr}^{51}$ labelled erythrocytes can be injected easily in less than one minute. Therefore, observations can be made during the first few minutes after injection, followed by serial observations during the first hour. Samples are then taken at 2, 4, 6, 24 , and 48 hours.

The shape of the "disappearance curve" after the injection of preserved, labelled erythrocytes is tri-phasic, due to: (a) mixing time, (b) a "rapid" phase, and (c) a "slow" phase. The curve then becomes identical in slope with that seen after the injection of fresh cells.

A "destruction gradient" can be measured between the value at the end of mixing time and the value at the end of the "slow" phase, which usually occurs at about 48 hours.

The per cent non-viable cells is equal to the "destruction gradient." This has been confirmed as follows :

(a) Total circulating hemoglobin was determined in several individuals with $\mathrm{Cr}^{51}$, as described by Gray and Sterling. These same individuals were then given about $40 \mathrm{ml}$. of labelled erythrocytes from preserved blood, and the total circulating hemoglobin again calculated, using the end of mixing time as the value representing dilution. These two methods agreed within an average of $4 \%$, with a maximum difference of $7 \%$.

Radioactivity was measured as counts per minute per gram of hemoglobin. Thus any error inherent in using hematocrits was eliminated.

(b) $450 \mathrm{ml}$. aliquots of the same preserved blood as that used above were transfused into recipients whose total circulating hemoglobin had been previously determined as described above (Gray, S. J., and Sterling, J., J. C. I. 29, 1614, 1950.) The per cent survival was determined using Ashby counts. These values agreed within $10 \%$ of those calculated from the "destruction gradient."

\section{The Participation of the Erythrocyte in the Electrolyte} Changes Induced by Intravenous Salt Loading. BEvERLY T. Towery and Howard E. Morgan, Nashville, Tenn. (Introduced by Hugh J. Morgan).

Plasma and erythrocyte concentrations and urinary excretion of sodium, potassium, chloride and water were studied in 15 normal young adults before and after a 60-minute infusion of 5 per cent sodium chloride. A constant oral water intake, established prior to infusion, was continued throughout the period of observation. A comparison is made betwen the pre-infusion period of diuresis and the second post-infusion hour. Erythrocyte 
concentrations of electrolytes were determined by an indirect method.

The pre-infusion erythrocyte sodium concentration tended to vary inversely with the plasma sodium concentration, but a definite relationship has not been established. The initial plasma potassium was maintained within a narrow range despite varying red cell concentrations.

After the infusion, the changes in concentration of erythrocyte sodium and potassium bore a relationship to their initial level; high values decreased, low values rose. A correlation of the changes in erythrocyte sodium with the changes in erythrocyte potassium revealed that these ions moved in the same direction.

The change in potassium clearance was inversely related to the pre-infusion clearance of this ion and did not correlate with the change in plasma or erythrocyte concentration thereof.

The rise in chloride clearance was closely correlated with the rise in sodium clearance, but was not related to changes in plasma chloride concentration. The alteration in sodium clearance varied inversely with the change in plasma sodium concentration. This appeared to be the result of cellular and renal changes rather than the cause. A direct relationship of questionable statistical significance was found between the changes in erythrocyte sodium concentration and increments in sodium clearance. These data suggest that cellular ionic exchanges, similar to those exhibited by the erythrocyte, are important in the regulation of the renal clearance and plasma concentration of sodium.

\section{$A$ Comparison of the Metabolism of $R b^{\mathrm{se}}$ and $K^{\mathrm{s}}$ Follow-} ing Simultaneous Injection into Man. Malcolm P. Tyor and JAMES S. Eldridge, Oak Ridge, Tenn. (Introduced by George T. Harrell).

The physical characteristics of $K^{2}$ ( $t 1 / 212.4 \mathrm{hr}$ ) have limited its widespread use.

Studies indicate that rubidium may replace potassium in several biological systems. The physical characteristics of $\mathrm{Rb}^{\text {क }}$ ( $t$ 1/2 $19 \mathrm{~d}$ ) are suitable for tracer studies in man.

Simultaneous intravenous injections of $K^{\mathbf{s}}$ and $\mathbf{R b}^{\text {*0 }}$ were given to four patients with leukemia and carcinoma. Stable rubidium injected varied between $0.25 \mathrm{meq}$ and 1.52 meq to determine any effect of "carrier"; potassium injected was 0.07 to 1.04 meq.

Multiple samples of plasma, red cells, and urine were obtained from all patients; white cells were separated in one patient. Each sample was assayed in a well scintillation counter. Counts of $\mathrm{Rb}^{* \boldsymbol{s}}$ and $\mathrm{K}^{\mathrm{s}}$ were determined by taking advantage of their half lives, and analyzed statistically.

The per cent of total counts of $\mathrm{Rb}^{20}$ and $\mathrm{K}^{23}$ in each sample of plasma, red cells, and urine was relatively constant within each patient. When the ratio of $\mathrm{Rb}^{*}$ to $\mathrm{K}^{\text {s" }}$ in the plasma and red cells of individual patients was compared to the ratio of the mixture injected, they were found to be similar. The ratio for white cells also agreed closely with that of the administered dose.

In contrast, the ratio of $\mathrm{Rb}^{\mathrm{w}}$ to $\mathrm{K}^{\mathrm{s}}$ in the urine of each patient was constantly lower than in the mixture injected. The lower values, however, bore a consistent relationship to the ratio of the dose injected in each patient. The ratios of per cent total counts $\mathrm{Rb}^{\text {ec }}$ in urine to the per cent total counts $\mathbf{R b}^{\mathbf{*}}$ injected were similar in all patients.

Within individual patients the data indicate a constant ratio of $\mathrm{Rb}^{26}$ and $\mathrm{K}^{23}$ in plasma, red cells, white cells, and urine. The ratio is similar to that of the injected dose in all fractions except urine, which showed less $\mathrm{Rb}^{\text {es }}$ and more $\mathrm{K}^{\mathrm{s}}$.

Studies of Serum and Gastric Factors in the Etiology of Pernicious Anemia: A Proposed In Vitro Test for Intrinsic Factor Activity. Walter G. Unglaub and O. Neal Mirler, New Orleans, La. (Introduced by Grace A. Goldsmith).

Normal human gastric juice, collected following histamine stimulation, markedly enhances the vitamin $B_{12}$ combining activity of serum when mixtures of serum, gastric juice, and added free vitamin are incubated together. This effect depends upon the ratio of gastric juice to serum, as well as the initial concentration of the added free vitamin, and is greatly in excess of the enhancement predicted for simple mixtures. Gastric juice from patients with pernicious anemia in relapse or in remission possesses this potentiating activity to a much smaller extent than does an equal volume of gastric juice from normal persons.

Incubation of normal gastric juice with serum from patients with pernicious anemia does not potentiate the $B_{12}$ combining activity as much as incubation with normal serum. Gastric juice from patients with pernicious anemia enhances the combining activity of pernicious anemia serum only slightly when compared with normal gastric juice.

Studies in one patient with pernicious anemia before and after adequate oral therapy with a commercial preparation containing vitamin $B_{12}$ and an intrinsic factor concentrate suggest an improved in vitro combining activity of the serum following therapy.

These observations lend support to the concept of the participation of a "serum factor" in the etiology of pernicious anemia. The techniques employed in this study have been applied to the in vitro evaluation of the antipernicious anemia activity of various intrinsic factor concentrates and they appear to give a valid test for intrinsic factor activity. Megaloblastic anemias other than pernicious anemia are being investigated with this technique.

The Relationship of Leukocyte Alkaline Phosphatase to Pituitary-Adrenal Activity. William N. Valentine,* James H. Follette, Barton E. Hardin and William S. BECK, Los Angeles, Calif.

Unit cell leukocyte alkaline phosphatase activity frequently increases several fold in the presence of such diverse circumstances as infection, trauma, vascular emergencies, surgery and diabetic acidosis in which pituitary-adrenal hyperactivity may be a common de- 
nominator. In patients studied before and after surgery leukocyte phosphatase uniformly rose stepwise to peak values averaging four times the pre-surgery level 72 hours after the procedure, remained elevated in the presence of complications, and returned to baseline over the next several days in uncomplicated procedures. A similar four fold increase occurred in each of five subjects receiving Acthar Gel for a 72 hour period with return to normal by 72 hours after stopping medication. Oral and intramuscular cortisone produced no or small responses over a similar period. Phosphatase activity required at least 72 hours to become maximally elevated in response to either a relatively prolonged "stress" or ACTH medication, appearing to increase rather uniformly and continuously during this time interval. Of considerable interest has been the observation that in all cases of active rheumatoid arthritis and disseminated lupus erythematosus and in most cases of uncomplicated acute rheumatic fever thus far studied the leukocyte phosphatase activity has not been increased. This suggests that in these diseases and in these patients this form of "stress" may not result in increased endogenous pituitary and/or adrenal activity. These data are not adequately explained by alterations in the leukocyte differential formula in subjects studied. Further investigation anticipates exploration of time-dose relationships and the effect of other steroids.

\section{Persistent Neutropenia with Maturation Arrest of the Bone Marrow in Graves's Disease, Not Related to Drugs. W. P. VanderLaan and Henry W. Cohen, Boston, Mass. (Introduced by E. B. Astwood).}

A woman presented classic features of Graves's disease, untreated, and a leukopenia. There were 3,100 leukocytes per cu. mm., $6 \%$ polynuclear neutrophils, $30 \%$ monocytes, $60 \%$ lymphocytes, $2 \%$ eosinophils, and $2 \%$ basophils. Bone marrow examination disclosed normocellularity, slight lymphocytosis, and maturation arrest at a promyelocyte level. Eosinophils were mature; there was no erythroid or megakaryocytic abnormality. The hematologic findings remained unchanged during one month during which there was no treatment. For two months after radioactive iodine treatment the hyperthyroidism and leukopenia persisted; the protein-bound iodine level was $15.6 \mu \mathrm{g} . / 100 \mathrm{ml}$. In the next month both the hyperthyroidism and the leukopenia improved. Lymphocytosis, monocytosis, leukopenia, and anemia have been described in Graves's disease; the present case suggests that neutropenia approaching agranulocytosis may also be associated with thyrotoxicosis. Search disclosed no evidence of exposure to chemical agents known to suppress myelopoiesis.

Two-hundred and fifty ml. of the patient's plasma, taken after some improvement had occurred, was infused into a normal 42-pound boy and induced no significant change in his peripheral blood.

The case illustrates persistent neutropenia paralleling the severity of Graves's disease, not relating to drugs.
Turn-Over Rates of Various Plasma Proteins in Normal and Cirrhotic Humans Using Biosynthetic Methods. Wade Volwiler,* Patrick D. Goldsworthy, Marion P. MacMartin, Patricia Ann Wood, Ian R. Mackay, Kenneth Fremont-Smith and Dorothy F. Shook, Seattle, Wash.

With S-35 1-cystine, two contrasting biosynthetic techniques have been employed to measure turn-over rates of specific plasma protein fractions in normal subjects and in edema-free patients with advanced portal cirrhosis: (1) oral single administration of labeled amino acid precursor with subsequent serial sampling of plasma proteins for measuring rate of isotope decline, (2) transfusion of fresh normal pre-labeled donor plasma into suitable recipients, followed by measurement in the recipient of rate of loss of isotope from the infused proteins.

A total of 30 such determinations for specific protein fractions have been made, including albumin, $\beta_{1}$ lipoprotein and fibrinogen. Albumin has been prepared by the Batchelor-Brown modification of Cohn's Method 10, $\beta_{1}$ lipoprotein by Cohn's Method 10 with subsequent ultracentrifugation (Gofman), and fibrinogen by the method of Surgenor et al. The composition of these fractions from each experimental subject has been repeatedly appraised by electrophoresis, and homogeneity has varied from $80-100 \%$.

Following are the average values of half-life (days) obtained for each fraction with biosynthetic techniques (1) and (2) : Albumin: Normal (1) 29 (2) 20, Cirrhosis (1) 21 (2) 24 ; Cohn I + III : Normal (1) 7, Cirrhosis (1) $10 ; \beta_{1}$ lipoprotein: Normal (1) 4.5 (2) 3.5 , Cirrhosis (1) 7.7 (2) 5; Fibrinogen : Normal (1) 5 (2) 4, Cirrhosis (2) 4.

These data suggest: (1) Re-utilization of isotope does not produce error in measurement of half-lives with biosynthetic method I, (2) An abnormal size in the circulating mass of a specific plasma protein is not necessarily associated with an abnormal life-span, (3) Iodinization of plasma proteins may sufficiently denature protein as to result in a shortened life-span.

Evidence for a Qualitative Difference Between the Gamma Globulin of Patients with Multiple Myeloma and of the Normal Human. RoBert L. Wall and LiLla Shu Yung Sun, Columbus, O. (Introduced by Charles A. Doan).

Many investigators have postulated that the hypergammaglobulinemia of some multiple myeloma patients represents a protein that differs qualitatively from normal gamma globulin. Proof that such a difference exists has been lacking. In the present study evidence is presented to support such a qualitative difference.

Gamma globulin preparations of adequate homogeneity could not be obtained with existing fractionation procedures. Therefore, a method was developed employing zinc diglycinate as a precipitating agent with subsequent chelation of the protein-bound zinc by the sodium salts of ethylene-diamine-tetra-acetic acid. This method rou- 
tinely produces an extremely homogeneous gamma globulin preparation as judged by the following tests: electrophoresis (both zone and boundary methods) with calculation of heterogeneity constants; optical ultracentrifugation with calculation of sedimentation constants; and by quantitative precipitin experiments employing specific rabbit antiserums. The method is applicable both to normal plasma and to plasma from myeloma patients.

Gamma globulin prepared by this method from pooled normal plasmas and from myeloma plasmas was then utilized for the production of rabbit antiserums. Previous quantitative precipitin experiments by the present authors, as well as other investigators, have shown that normal gamma globulin behaves as an immunologically homogeneous protein. Quantitative precipitin experiments with the present normal gamma globulin antiserum show marked differences in the precipitin curves when normal globulin and myeloma gamma globulin are used comparatively as antigens, as would occur if these proteins differed qualitatively. The reaction of myeloma gamma globulin antiserums with normal gamma globulin showed the presence of some normal gamma globulin in the myeloma gamma globulin. However, after maximal precipitation of the myeloma antiserum by addition of normal gamma globulin, this antiserum is still capable of precipitation with myeloma gamma globulin, demonstrating a true qualitative difference between normal and myeloma gamma globulin.

Hepatic Coma: Effect of Sodium Glutamate. LESLIn T. Webster, Jr. and Charles S. Davidson,* Boston, Mass.

Ammonia or related compounds have been implicated as possible etiologic factors in the production of hepatic coma. The highest blood and plasma "ammonia" concentrations have been found in this condition. The syndrome of impending hepatic coma can be produced in some patients with hepatic disease by administration of ammonium salts. Glutamic acid plays an important role in intermediary nitrogen metabolism and can combine with ammonia to form less toxic compounds. Walshe reported favorable results with this amino acid in the treatment of hepatic coma.

In the present study 23 grams of sodium glutamate was given intravenously to eight patients with Laennec's cirshosis who were in coma or impending coma. $\mathrm{Pa}$ tients evaluated daily with respect to state of consciousness and the characteristic "flapping" tremor were separated into three groups: coma, impending coma, and impending coma induced by ammonium salts. Three patients were studied on more than one occasion and in more than one group. Plasma "ammonia" concentrations were measured.

Two comatose patients showed questionable temporary improvement, one after three isolated infusions and the other after four daily infusions of glutamate; both subsequently died. Six patients with spontaneous impending coma received from one to three daily infusions of glutamate; again, with little, if any, improvement. Four in this group subsequently expired. Impending hepatic coma was induced in two patients by administration of ammonium salts. Glutamate failed to modify this syndrome which, however, disappeared after discontinuing ammonium salts. One of these patients was then given glutamate prior to ammonium salt administration, but this amino acid failed to prevent the onset of the characteristic syndrome.

Thus, sodium glutamate was found to have little, if any, beneficial effect on liver coma or impending coma; it also failed to prevent onset and exacerbation of impending coma induced by ammonium salts. Plasma "ammonia" concentrations showed no consistent changes.

Comparative Incorporation of $S^{25} l$-Cystine and $S^{\text {st }}$ Sodium Sulfate by Normal and Leukemic Leukocytes. Austin S. Weisberger, Letr G. Suhrland and BenNetr Levine, Cleveland, $O$. (Introduced by T. $H$. Ham).

Previous studies indicate that $l$-cystine (or $l$-cysteine) may have an important role in leukocyte metabolism. Comparison of the uptake of $S^{25}$ sodium sulfate with that of $S^{25}$-cystine by normal and leukemic leukocytes reveals certain differences in incorporation both in vivo and in vitro.

Tracer amounts of $S^{25}$ l-cystine or $S^{25}$ sodium sulfate were administered orally to normal and leukemic patients. The concentration of $\mathrm{S}^{25}$ per $\mathrm{ml}$ packed leukocytes was determined at intervals. In vitro studies were obtained by incubating whole blood with these compounds and measuring the radioactivity incorporated per $\mathrm{ml}$ packed leukocytes.

Characteristic patterns of incorporation were obtained for orally administered $l$-iystine in normal and leukemic leukocytes. Acute leukemic leukocytes incorporated $l$-cystine most rapidly. The disappearance curves of radioactivity indicate that the approximate life span of leukocytes in normals is 13 days, in chronic myeloid leukemia 8 days, in acute leukemia 3 days and in chronic lymphatic leukemia longer than the period of observation.

Orally administered $\mathrm{S}^{25}$ sodium sulfate is utilized poorly by leukocytes in acute leukemia and in chronic lymphatic leukemia. However, incorporation of sodium sulfate by normal and chronic myeloid leukemic leukocytes is comparable to that of orally administered $l$-cystine.

$l$-cystine is readily incorporated by both normal and leukemic leukocytes in vitro. This is an active process and not solely dependent upon simple diffusion. In contrast, however, only chronic myeloid leukemic leukocytes readily incorporate sodium sulfate. All other types of leukocytes exhibit a low level of incorporation of sodium sulfate.

The data demonstrate differences in utilization of $l$-cystine and inorganic sulfur by normal and leukemic leukocytes. The findings are compatible with the apparently unique role of $l$-cystine in leukocyte metabolism. 
A Study of Renal Tubular Phenomena Under the Infuence of a Carbonic Anhydrase Inhibitor. L. G. Wert,* D. T. Young, O. A. ThoruP, JR. and C. H. Burnetr,* Chapel Hill, N. C.

The consequences of the administration of a carbonic anhydrase inhibitor were studied in three normal male subjects in an experimental setting characterized by a modest acidosis and a state of physiologic diabetes insipidus. The former was induced by the ingestion of ammonium chloride for five days, and the latter by the administration of an infusion of a dilute solution of glucose in water in a manner designed to achieve and maintain a positive balance of water of a liter or more. The carbonic anhydrase inhibitor promoted: 1) a striking increase in the rates of excretion of water, sodium, bicarbonate, and total solutes; 2) lesser increases in the rates of excretion of potassium and chloride; 3 ) decreases in the rates of excretion of ammonia and titratable acid;4) an increase in the $\mathrm{pH}$ of the urine; and 5) a slight decrease in the clearance of inulin.

An increase in urine flow (despite a decrease in GFR) superimposed on a state of physiologic diabetes insipidus implies that a larger volume of fluid had gained access to the distal tubule. According to current concepts this would result from a decrease in active proximal reabsorption of solutes which, in this instance, were predominantly sodium and bicarbonate. Since these alterations were promoted by the administration of carbonic anhydrase inhibitor and the reabsorption of bicarbonate appears to be dependent on the secretion of hydrogen ions, the inference is that hydrogen ions are usually secreted in the proximal as well as the distal tubule.

The increase in urine flow was composed of increments of both "free" and "osmolar" water. These data can be explained as the result of an augmented distal tubular reabsorption of solutes consequent to the delivery of a larger volume of isosmotic fluid to this portion of the nephron.

SPCA-Induced Thrombosis: Its Pathogenesis and the Effect of Anticoagulants. Stanford Wesslar, Boston, Mass. (Introduced by Herrman L. Blumgart).

The pathogenesis of thromboembolism and the relative efficacy of drugs in the prevention of intravascular coagulation remain poorly defined. In this laboratory an experimental method has been developed whereby, in dogs, the systemic infusion of serum fractions has routinely produced venous thrombosis under conditions of simple stasis. The role of anticoagulants in inhibiting these thrombi has also been investigated.

Serum fractions prepared by barium sulfate adsorption and citrate elution are rich in SPCA (convertin) and its precursor, pro-SPCA (proconvertin) but are devoid of significant amounts of prothrombin, thrombin, thromboplastin and Ac-globulin. Antecubital vein infusions of these SPCA preparations routinely induced rapid thrombus formation in jugular vein segments completely isolated immediately after infusion, although no measurable rise in pro-SPCA or SPCA was demonstrable. Direct venous narrowing or external application of tourniquets to intact extremities immediately after SPCA infusions similarly induced coagulation distal to the obstructed areas.

Dicumarol was given to ten animals with consequent reduction of one-stage prothrombic activity to two percent of normal or below. In only one dog was SPCAinduced thrombosis prevented; in this animal the coagulation defect was so severe that the clotting time had been tripled. In striking contrast, heparin administered to ten animals in amounts sufficient to double the clotting time produced complete inhibition of SPCA-induced clots in all instances.

Excessive SPCA activity has been observed in some clinical states and its possible relation to thromboembolism has been proposed. The studies herein described provide experimental evidence for the concept that minute amounts of active SPCA (contrasted with inactive proSPCA) may under conditions of simple stasis initiate extensive intravascular clotting. The data also demonstrate that SPCA-induced thrombi are more effectively inhibited by heparin than by Dicumarol even when the latter drug produces a profound reduction in the circulating SPCA complex.

Metabolic Studies Demonstrating Increased Resistance of Hyperthyroid Patients to Pitressin Tannate Administration. Raymond E. Weston,* Helen B. Horowitz, Shirley Rubler and Jacob Grossman, New York, N. Y.

Previous studies have established that intramuscular administration of 1 unit of Pitressin Tannate-in-Oil (PTO) b.i.d. to normal subjects and patients in congestive failure, on adequate fluid intakes, leads to marked water retention, increased urinary total solute concentration (U.T.S.C.), and hyponatremia. In contrast, we found that 5 units of PTO t.i.d. had no metabolic effect in a severely hyperthyroid woman without demonstrable renal disease, maintained on a high salt diet.

Since then, three additional hyperthyroid women on our metabolic ward have been given PTO for 6-12 days. To evaluate the influence of urinary solute load, two were maintained on a high (175 m.Eq.) and one on a low (25 m.Eq.) sodium intake. To compensate for hyperidrosis, fluid intakes were considerably increased.

All exhibited resistance to Pitressin. On 2 units t.i.d., one patient on high salt intake exhibited transitory increase in calculated U.T.S.C. from $366 \mathrm{~m} . O \mathrm{sm} . / 1$. to $450-520 \mathrm{~m}$. Osm./1., without change in urine volume (1800$2400 \mathrm{ml}$.) or serum electrolyte concentrations. Because of severe hyperidrosis, another on high salt intake had control urine volumes of $1400-1600 \mathrm{ml}$. with measured U.T.S.C. of 564-608 m.Osm./1. On PTO (2 units t.i.d.) her U.T.S.C. rose slightly to $650-700 \mathrm{~m} .0 \mathrm{sm} / 1$, and her serum sodium fell $7 \mathrm{~m} . \mathrm{Eq} . / \mathrm{l}$., partly due to increased natriuresis on the sixth and last day. The following day, 
her urine volume increased to $2200 \mathrm{ml}$., U.T.S.C. dropped transiently to $390 \mathrm{~m} . O s m . / 1$, weight fell $1 \mathrm{kgm}$., and serum electrolytes returned to normal. The one patient on the low sodium intake exhibited only slight antidiuretic response to PTO (2 units t.i.d.), as indicated by small U.T.S.C. increases from $285-355 \mathrm{~m}$.Osm./1. to $365-420$ m.Osm./1., with some water retention and subsequent natriuresis and hyponatremia.

Possible mechanisms responsible for the apparent resistance of these hyperthyroid patients to the anti-diuretic effect of PTO will be discussed.

Clinical Studies on Staphylococcal Septicemia. RoBert I. Wise and Caroline Cranny, Minneapolis, Minn. (Introduced by Wesley W. Spink).

Fifty-one cases of severe staphylococcal septicemia were studied and treated at the University of Minnesota and associated hospitals from November, 1951, through February, 1954. Twenty-one patients recovered, 27 expired and 3 are under treatment. The mortality rate, thus far, is $53 \%$. Sensitivity of the cultures of Micrococcus pyogenes var. aureus (Staphylococci) to the antibiotics was studied in vitro by the tube-dilution technique.

The percentages of cultures which were resistant to $\mathbf{5 0}$ units or micrograms per $\mathrm{ml}$. were as follows: penicillin $70.1 \%$, streptomycin $65.7 \%$, chlortetracycline $37.2 \%$, oxytetracycline $46.6 \%$, and chloramphenicol $2.5 \%$. The strains were sensitive to bacitracin, erythromycin, carbomycin and neomycin at concentrations of less than 10 units or micrograms per ml. in percentages of $92 \%, 100 \%$, $97 \%$, and $82 \%$ respectively. The percentages of cultures sensitive to one unit or microgram per $\mathrm{ml}$. were as follows: penicillin $17.1 \%$, streptomycin $2.4 \%$, chlortetracycline $21 \%$, oxytetracycline $23.1 \%$, chloramphenicol $0 \%$, bacitracin $26.2 \%$, erythromycin $87.8 \%$, and neomycin $34.2 \%$.

Bacteriophage typing of the cultures revealed $8.2 \%$ in group II, $44.5 \%$ in group III, $5.6 \%$ in a miscellaneous group (type $44 \mathrm{~A}$ ), none in group I, and $41.7 \%$ were nontypeable. Antibiotic-resistant cultures were prominent in group III and the non-typeable group. The percentages of group III strains resistant to 50 units or micrograms per $\mathrm{ml}$. were as follows: penicillin $87.5 \%$, streptomycin $75 \%$, chlortetracycline and oxytetracycline $43.7 \%$. The percentages of non-typeable strains resistant to 50 units or micrograms per ml. were as follows: penicillin $66 \%$, streptomycin $73 \%$, chlortetracycline $33 \%$ and oxytetracycline $53 \%$. Strains in group II and the miscellaneous group were sensitive or slightly resistant to all antibiotics. No correlation existed between the bacteriophage types and death of the patients.

Patients surviving were treated with combinations of antibiotics, which were selected on the basis of in vitro sensitivity tests. The combinations included penicillin and bacitracin, erythromycin and bacitracin, or carbomycin and bacitracin.
Oxygen Content of Pulmonary Artery Blood During the Respiratory and Cardiac Cycle in Relation to Possible Errors in Flow Measurement by the Fick Method. EARL H. Wood* and JohN T. ShEPhERD, Rochester, Minn.

Changes in blood flow and oxygen content associated with respiratory or cardiac cycles may introduce large systematic errors in determinations of cardiac output (Visscher). To study this possibility, a method was devised for withdrawal of blood samples from the heart and great vessels during any phase of respiratory or cardiac cycles. This consists of a synchronizer circuit controlling an electromagnetic valve interposed between cardiac catheter and blood-sampling syringe. Delay and hold circuits allow the synchronizer when activated by the onset of inspiration or expiration or by the $R$ wave of the ECG to hold the sampling valve open during any portion of respiratory or cardiac cycles. Oxygen saturation of blood withdrawn in this manner was determined by photometry and manometric methods. In 8 subjects without shunts (2 normal and 6 with mitral disease) there was no demonstrable difference in oxygen content of pulmonary artery blood during inspiratory or expiratory phases of the respiratory cycle. In 4 subjects (2 normals) there was no difference during systole and diastole. Failure to demonstrate differences means that if cyclic variations did occur, they were small. Hence, possible errors from this cause in cardiac output values by conventional Fick methods were also probably small.

Phasic differences in oxygen content of pulmonary artery blood were not demonstrated in 4 patients with large left-to-right shunts via atrial septal defects. However, small but probably significant changes in pulmonary artery samples with the cardiac cycle were found in some patients with patent ductus arteriosus or ventricular septal defect. The direction and magnitude of the systematic error in determination of pulmonary blood flow by the conventional Fick method under such circumstances is related to the product of the amplitude of the variations and the cosine of the phase angle between the variations in flow and oxygen content.

\section{Quantitative Fractionation of Serum Proteins Deter- mined by Moving Boundary and Paper Electrophoresis. Moses Wurm, New York, N. Y. (Introduced by Ernst P. Boas).}

Paper electrophoresis gives satisfactory qualitative separations of a variety of complex mixtures but the quantitative aspects of the method as applied to serum proteins have not been adequately examined. To obtain consistently reproducible electrophoretic patterns that lend themselves to quantitation it was essential to keep the filter paper strips freely suspended in a taut, horizontal position throughout the period of electrophoresis and subsequent drying. Staining either with bromphenol blue or Amido-Schwarz 10B gave similar values. The dry patterns were measured in a photoelectric densitometer (Photovolt 501A) using white light, and curves were obtained by plotting the antilog of the optical den- 
sity readings against distance of migration on the paper strip. This conversion expressed the observed proportionality, determined from calibration curves, between optical density and the logarithm of protein concentration.

Serum protein distributions thus determined compared favorably with the values obtained in a moving boundary apparatus (Aminco Portable). Ten pairs of consecutive determinations on the serum of a single donor by paper (P) and moving boundary (M) electrophoresis expressed in terms of relative protein concentrations gave the following mean results \pm S.E. : albumin (P) $58.5 \pm 1.29$ and (M) $59.1 \pm .67(\mathrm{t}=.41)$; alpha $\mathrm{a}_{1}$-globulin (P) $3.9 \pm .27$ and $(\mathrm{M}) 4.5 \pm .24(\mathrm{t}=1.39)$; alpha $_{2}$-globulin $(\mathrm{P}) 7.2 \pm$ .27 and $(M) 8.7 \pm .47(t=2.77)$; beta-globulin (P) 13.0 \pm .56 and $(\mathrm{M}) \quad 13.9 \pm .35 \quad(\mathrm{t}=1.36)$; gamma-globulin (P) $17.4 \pm .94$ and $(\mathrm{M}) 13.8 \pm .44(\mathrm{t}=3.46)$. Statistically, the albumin values showed a high degree of identity while the alpha, - globulin and beta-globulin values were at variance within experimental limits. The highly significant difference in gamma-globulin values might be attributed to protein adsorption on paper, an inherent characteristic of paper electrophoresis. The significant difference between the alpha $_{2}$-globulin values remains unexplained.

Excretion of Hydroxyproline in Patients with Rheumatic Diseases and in Children. MoRris ZIFF, ANDRE KIBRICK, Ellis Dresner and H. Joel Gribetz, New York, N. Y. (Introduced by Currier McEwen).

Hydroxyproline constitutes 15 per cent of collagen, and except for small amounts present in elastin, is found in no other protein of the body. The excretion of this amino acid was determined in patients with "collagen diseases" as well as in control subjects in order to determine whether differences due to abnormal collagen metabolism might be detected. Forty-five adults and fourteen children were placed on a standard low hydroxyproline diet. Free and total urinary oxyproline were determined by the colorimetric method of Wiss, which was shown to agree closely with a chromatographic, radioactive isotope derivatization method. Total amino acid, creatinine, and nitrogen were also determined.

Over 95 per cent of excreted hydroxyproline was in bound form. Excretion was not increased by administration of cortisone or by ingestion of 4 grams of oxyproline or proline daily, although there was a prolonged rise in blood level and in excretion of free hydroxyproline after ingestion of the latter. Hydrolysis studies suggest that the bound hydroxyproline of urine is in peptide linkage. Since this peptide is apparently not formed from administered hydroxyproline, it may represent a breakdown product of collagen.

No significant difference was observed between the excretion of hydroxyproline in normal adults and in a group of adult patients with miscellaneous diseases on the one hand, and patients with rheumatoid arthritis and other "collagen diseases" on the other, either on an absolute basis or as per cent of total amino acid. Thus no significantly increased breakdown of collagen in patients with diseases of the connective tissue was demonstrated.

The excretion of hydroxyproline in children was two to three times greater than in adults on an absolute basis, and four to five times greater when expressed as per cent of total amino acid. Increased excretion of bound hydroxyproline by children may indicate the presence of an increased pool of hydroxyproline peptide for collagen synthesis in the growing individual. 